\title{
Optical biopsy in the anterior segment of the eye
}

Citation for published version (APA):

Hosseini, K. (2008). Optical biopsy in the anterior segment of the eye. [Doctoral Thesis, Maastricht University]. Maastricht University. https://doi.org/10.26481/dis.20081212kh

Document status and date:

Published: 01/01/2008

DOI:

10.26481/dis.20081212kh

Document Version:

Publisher's PDF, also known as Version of record

\section{Please check the document version of this publication:}

- A submitted manuscript is the version of the article upon submission and before peer-review. There can be important differences between the submitted version and the official published version of record.

People interested in the research are advised to contact the author for the final version of the publication, or visit the DOI to the publisher's website.

- The final author version and the galley proof are versions of the publication after peer review.

- The final published version features the final layout of the paper including the volume, issue and page numbers.

Link to publication

\footnotetext{
General rights rights.

- You may freely distribute the URL identifying the publication in the public portal. please follow below link for the End User Agreement:

www.umlib.nl/taverne-license

Take down policy

If you believe that this document breaches copyright please contact us at:

repository@maastrichtuniversity.nl

providing details and we will investigate your claim.
}

Copyright and moral rights for the publications made accessible in the public portal are retained by the authors and/or other copyright owners and it is a condition of accessing publications that users recognise and abide by the legal requirements associated with these

- Users may download and print one copy of any publication from the public portal for the purpose of private study or research.

- You may not further distribute the material or use it for any profit-making activity or commercial gain

If the publication is distributed under the terms of Article $25 \mathrm{fa}$ of the Dutch Copyright Act, indicated by the "Taverne" license above, 


\title{
Optical Biopsy in the Anterior Segment of the Eye
}

\author{
PROEFSCHRIFT \\ ter verkrijging van de graad van doctor \\ aan de Universiteit Maastricht, \\ op gezag van de Rector Magnificus, \\ Prof. mr. G.P.M.F. Mols \\ volgens het besluit van het College van Decanen, \\ in het openbaar te verdedigen \\ op Vrijdag, 12 December 2008 om 10 uur \\ door \\ Kamran Hosseini \\ Geboren op 24/12/1964 \\ Te Teheran
}




\author{
Promotores: \\ Prof. Dr. F. Hendrikse (Universiteit Maastricht) \\ Prof. Dr. M. Motamedi (University of Texas Medical Branch, Galveston, Texas) \\ Copromotor: \\ Dr. R.J. Erckens (Universiteit Maastricht)

\section{Beoordelingscommissie:} \\ Prof. dr. Steinbusch (voorzitter) \\ Prof. dr. Bruggeman \\ Prof. dr. ir. van Gemert, Lasercentrum, Academisch Medisch Centrum \\ Dr. ir. Puppels, Erasmus Medisch Centrum Rotterdam \\ Prof. dr. Ramaekers
}

This work was performed at the Center for Biomedical Engineering and Department of Ophthalmology (Professor M. Motamedi, Ph.D.) at the University of Texas Medical Branch, Galveston, Texas, USA, and was sponsored in part by a grant from "Research to Prevent Blindness Inc." 
The intricacy and seeming perfection of the eye, both as an object of anatomical beauty and as a working machine, have worried and intrigued scientists, philosophers and zealots, especially during the intellectual upheavals of the nineteenth century. The eye is a complex sensory receptor in the body, which is specialized for the gathering of visual information. The eye gives us the sense of sight, allowing us to learn about the world more than any other sense. A substantial amount of everything we learn is perceived through our eyes.

To Delnaz and Fariba 


\section{Contents}

Chapter 1- Introduction $\quad 5$

-The continuous need for vision research 5

-Investigate two different forms of light backscattering to perform optical biopsy 6

-Non-invasive examination of the eye $\quad 7$

-Anatomy and physiology of the anterior segment of the eye 10

-Eye and drug interventions 11

$\begin{array}{ll}\text {-Spectroscopy } & 11\end{array}$

-Optical biopsy using light backscattering $\quad 12$

-Technical aspects $\quad 12$

-Raman spectroscopy $\quad 12$

-General applications of Raman spectroscopy 15

-Applications of Raman spectroscopy in ophthalmology 15

-Optical coherence tomography 16

-Applications of OCT in ophthalmology $\quad 19$

-Axial scatter intensity mode with OCT 20

-Aim of thesis 21

$\begin{array}{ll}\text {-References } & 22\end{array}$

Chapter 2 - Noninvasive detection of Ganciclovir in ocular tissue by Raman spectroscopy: implication for monitoring of drug release 26

Chapter 3 - Noninvasive monitoring of commonly used intraocular drugs

against Endophthalmitis by Raman spectroscopy 39

Chapter 4 - Raman spectroscopy: noninvasive determination of silicone oil

in the eye: potential applications for intraocular determination of biomaterials

Chapter 5 - In vivo OCT assessment of rabbit corneal hydration and dehydration

Chapter 6 - Accurate in vivo measurements of the corneal light backscattering in-depth distribution with OCT for different hydration states

Chapter 7 - Monitoring of rabbit cornea response to dehydration stress by optical coherence tomography

Chapter 8 - Noninvasive monitoring of water movement through rabbit cornea using optical coherence tomography

$\begin{array}{lr}\text { Chapter } 9 \text { - Summary and conclusions } & 127\end{array}$

$\begin{array}{ll}\text { Few words of appreciation } & 140\end{array}$

$\begin{array}{ll}\text { Publications and presentations } & 141\end{array}$ 


\section{Chapter 1}

\section{Introduction}

\section{The continuous need for vision research}

The World Health Organization (WHO) estimated in 2002 that 161 million people or $2.6 \%$ of the world population were visually impaired, of whom 124 million (about 2\%) had low vision and 37 million (about $0.6 \%$ ) were blind. Low vision is a term that denotes a level of vision that is less than $30 \%$ of normal vision and cannot be fully corrected with conventional glasses. The main causes of world wide low vision and ultimately blindness are cataract, glaucoma, corneal scarring (from a variety of causes), age-related macular degeneration, and diabetic retinopathy.

According to the National Eye Institute, as the nation ages in the United States more Americans than ever before are facing vision problems from eye disease. By the year 2030, twice as many people will be blind as today. These growing numbers are indicating the need for continuous research in the ophthalmic and visual sciences.

Vision impairment has a significant impact on the length and quality of life.

Previous research has shown that vision impairment is associated with an increased risk of falls, hip fractures, depression, social isolation, greater need for community services, and greater risk of admission to nursing homes ${ }^{1-7}$. As the global population ages and the average lifespan lengthen, more people will be faced with the challenge of adapting to vision loss. This will increase the demand for eye-care and vision-related rehabilitation programs and services. The provision of these services is costly and to some extend, unforeseen.

When vision impairment is recognized early, treatment can be more effective, enabling people to preserve their independence longer while this can also add to the length of their life expectancy. Seeking independence is apparent as more senior citizens, for example ask the clinicians to approve and enable them for continuous driving eligibility.

Recognition of a disease and ultimately the establishment of correct diagnosis form the mainstay of any treatment. In modern medicine tissue sampling in the form of a biopsy is utilized and this requires physical removal of tissue or cells from the organ. These specimens are examined under a microscope and can be analyzed chemically to obtain information at the molecular level. A biopsy implies risks such as bleeding, spreading of infections, and tumors. Smaller organs such as the eye are less suitable to lend their highly sensitive structures for an invasive biopsy method. Furthermore, while a biopsy can serve to determine the pathology in a tissue, it usually cannot monitor a physiological or a therapeutic induced change in real-time. 
Optical biopsy is a more recent development of the biopsy technique. The main advantages of optical biopsy are that the tissue is scrutinized in situ and that the tissue is neither damaged nor destroyed. This approach is still young and in this thesis the feasibility of this type of biopsy in the anterior segment of the eye using different backscattering properties of light is shown.

\section{Investigating two different forms of light backscattering to perform optical biopsy}

Two new non-invasive diagnostic modalities, "Raman Spectroscopy" and "Optical Coherence Tomography" (OCT), have been recently introduced in the field of ophthalmology. Raman spectroscopy is an example of inelastic scattering of light and its application so far in ophthalmology has been mostly an experimental diagnostic technique. Due to the required amount of potentially hazardous light to the eye, it remained for a long time a challenge to justify its clinical application. Erckens ${ }^{8,9}$ and $\mathrm{Smit}^{10}$ demonstrated some promising clinical application possibilities for Raman spectroscopy. More recently successful employment of Raman spectroscopy by Bernstein ${ }^{11}$ to measure Lutein and other substances in the living human eyes has reaffirmed the value of this technology for the clinic. Hence, additional applications of this technology can be expected. OCT on the other hand, is an example of elastic scattering of light and it has already been implemented in standard clinical examination. So far its application is mainly focused on detection of a defect and/or collection of fluid within various ocular structures especially in the retina. OCT is not only a valuable imaging technique but it can also be used as an optical biopsy instrument to quantify pathology and/or a defect. The aim of these studies is to explore potential possibilities of these two different optical techniques. Raman spectroscopy can be used in backscatter mode, while OCT always operates in backscatter mode. Both techniques can operate in an imaging mode. However, the imaging mode for Raman is time consuming and requires more energy than is safe for the eye. In order to compare both techniques in ophthalmology, they are used in a so-called A-scan mode analogue to ultrasound. Currently OCT is applied in a B-scan (imaging) mode. In contrast to Raman spectroscopy, OCT only gives quantitative information when applied in A-scan mode. With a priori knowledge of the molecular composition of the tissue however, the A-scan can also be interpreted qualitatively. This comparison is part of the aim of this thesis. Enhancements have been introduced to both techniques to increase the signal to noise ratio substantially. For instance, in case of the Raman spectroscopy technique, binning of the chip has been applied to increase the detection limit of different solvents at lower concentrations. 
For OCT, the averaging technique has been incorporated to substantially reduce the amount of "speckle noise" in order to detect the real change in the infrared backscattering. Speckle noise in OCT is the noise generated in an optical fiber system by the combination of mode-dependent optical losses and fluctuation in the distribution of optical energy among the guided modes or in the relative phases of the guided modes.

\section{Non-invasive examination of the eye}

The ideal way of acquiring information from the eye should be based on noncontact, non-invasive and real-time techniques. Optical based diagnostic and research techniques enable acquisition of data that remains less affected by potential "methodsrelated" artifacts, which are generally inevitable by (minimally-) invasive methods. At the same time, they warrant the practical application of the investigated method in patient care. The importance of this facet of the eye research has been recognized and so optical, acoustic, and radiological based techniques have emerged among the logical choices for the involved vision scientists.

The eye is the only organ in the body, which provides a true optical window to the internal environment of the body. Moreover, the eye is an organ that reacts significantly to a small amount of light stimulus (e.g. pupil reaction). These properties are among the fundamental rationales for the appreciated use of optical based procedures and devices by ophthalmologists. Developments of modern optical techniques have broadened the arsenal of the available tools for sensing and measurements of the eye and eye related parameters. Optical techniques such as fundoscopy, keratometry, slitlamp biomicroscopy, and refractometry belong to the available group of diagnostic tools for ophthalmologists and vision scientists. These techniques not only provide information about the health of the eye but may also provide information on conditions elsewhere in the body that may affect the eye and/or other organs.

The two optical systems used in this thesis are Raman spectroscopy (RS) and Optical Coherence Tomography (OCT), which were employed to investigate different forms of light backscattering that could provide information about the internal environment of the anterior segment of the eye. 


\section{Anatomy and physiology of the anterior segment of the eye}

Figure 1. The anatomy of the eye

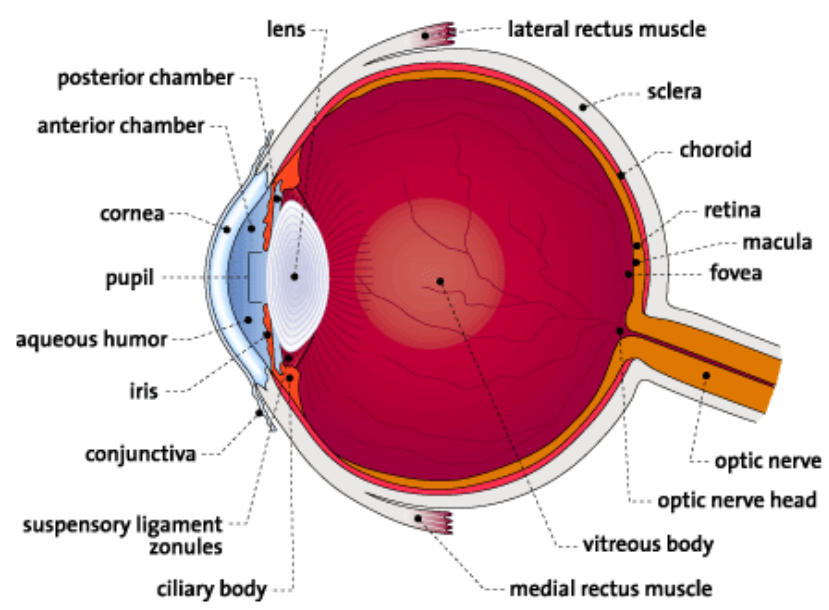

The anterior segment of the eye is composed of the cornea, anterior chamber, iris and the lens. The anterior chamber is the area bounded in the front by the cornea and in the back by the lens, and is filled with aqueous humor. Different attributes such as ideal transparency, a favorable location in front of the eye and separated from the outside world only by the cornea, this region provides an excellent window for optical biopsy. The aqueous humor (the fluid which fills the anterior chamber) is produced by the epithelium of the corpus ciliary and its main ingredients in terms of the biochemical composition are in relative balance and certain correlation with the blood plasma. Molecules such as glucose, lactate, certain amino acids and urea are present in this medium $^{12}$. Studies have shown that systemically injected drugs also appear after a certain time delay within the aqueous humor ${ }^{13-15}$. Other investigators have also shown that the intravitreally-injected substances can appear in the aqueous humor ${ }^{16,17}$. The same holds true for the topically (mostly lipophilic) administrated drugs in the eye. These drugs, once circulating and present within the anterior chamber of the eye, could potentially affect the morphology and physiology of the bordering structures such as the lens, cornea and iris ${ }^{18-20}$. The aqueous humor in the anterior chamber is the main source for the well-balanced corneal hydration ${ }^{21}$. There is a constant movement of water between the anterior chamber and the stroma of the cornea through its endothelial layer. This water transport takes place in both directions using both passive and active mechanisms and is crucial in maintaining corneal transparency. The possibilities for a non-invasive optical monitoring of water transport across the cornea will be discussed in this thesis. 


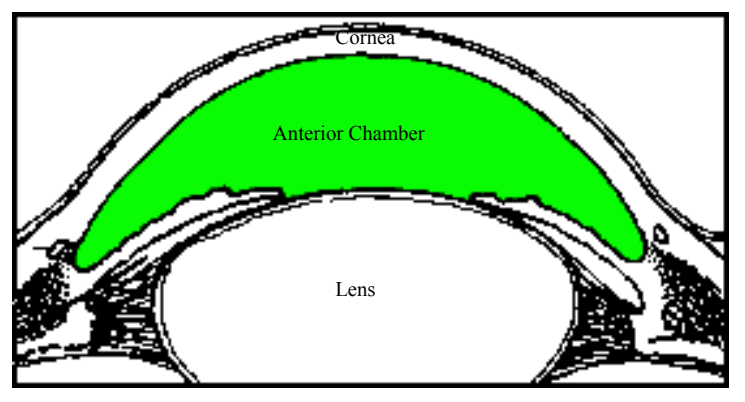

Figure 2. Anterior chamber filled with the aqueous humor (gray area between the cornea and the lens).

\section{Eye and drug interventions}

Multiple pathological conditions of the eye require certain drug interventions depending on the severity of the disease and availability of medication. The administration of drugs in the eye can be achieved via different routes. These include systemic, topical, and intravitreal routes. In recent years, other means have also been utilized for ocular drug delivery. Some examples consist of intravitreal implants ${ }^{22,23}$, corneal contact lens drug delivery ${ }^{24}$, ocular iontophoresis, ${ }^{25}$ and other forms of transscleral drug delivery. Every approach is typically evaluated by its efficiency and practicality and is usually prescribed and administered by ophthalmologists. With the main goal as their therapeutic effect(s), application of drugs in the eye carries concerns such as the efficacy, bioavailability, toxic thresholds, general side effects, and a possible long-term secondary impact(s) on different ocular tissues such as the retina, iris, and the cornea. These issues emphasize the necessity for real-time data acquisition regarding the quantity of the drug in the eye and its possible secondary effects on the related physiology and morphology.

\section{Spectroscopy}

Spectroscopy is the study of light as a function of wavelength that has been emitted, reflected, or scattered from a solid, liquid, or gas. When photons go through a certain material, some are reflected from particle surfaces, some pass through the particles, and some are absorbed. Those photons that are reflected from particles surfaces or refracted through them are called "scattered". Scattered photons may encounter another grain or be scattered away from the surface so they may be detected and measured. The variety of light-matter processes and their wavelength dependence permit us to obtain information about the chemistry of the material (gas, solid, or liquid) from its absorbed, reflected, or emitted light by means of spectrometry. 
The human eye is among others a spectrometer itself: we can look at a surface and see color. A modern spectrometer, can measure fine details over a broad wavelength range and with great precision. Moreover, it can measure absorptions quantitatively.

\section{Optical biopsy using light backscattering}

One mode of data collection with an optical system is by using a specific property of light after irradiating a sample namely light scattering as briefly described above. The technologies used in this thesis are based on light backscattering. When electromagnetic radiation passes through optically transparent matter, most of the radiation continues in its original direction and only a small fraction is scattered in other directions. From the scattered part of the light, over $99 \%$ is scattered at the same wavelength as the incoming light such as Mie and Rayleigh scattering (example of "elastic" scattering), this is also known as "coherent scattering" since the scattered light is coherent with the incident light. In case of the Rayleigh scattering, the intensity is inversely proportional to the fourth power of the incident wavelength. This scattered light contains morphological information from the tissue. One of the systems capable of visualizing this scattering is Optical Coherence Tomography (OCT).

A smaller part of the light however, less than one percent, exchanges energy with molecules. Scattering due to such a collision is called "inelastic scattering". This light contains information about the molecular composition of the tissue. Raman spectroscopy can visualize these molecular compositions.

\section{Technical aspects}

Raman spectroscopy is performed with monochromatic light while in Optical Coherence Tomography a certain bandwidth of the light source is necessary to obtain the required spatial resolution (low coherence interferometry). Once the eye has been perturbed with a substance (e.g. drugs, hypertonic or hypotonic agents, water) each of these techniques can acquire a different aspect of information about the applied drugs and/or other surrounding substances and solutes such as water and glucose, their concentrations at a given point in time and space, and their possible impacts on the structures within the anterior segment and the associated physiology.

When we need chemical information of the eye, we may use "confocal" Raman spectroscopy. Raman spectroscopy provides information about the molecular composition of the target tissue and to detect and identify the presence of a certain molecule, the tissue is probed by light from a monochromatic light source. The Raman signal is about $10^{-6}$ to $10^{-8}$ of the scattered light. 
In order to reduce signals from adjacent tissues, a confocal setup needs to be used, which can isolate and analyze a small volume of tissue and thus perform optical biopsies (See figure 3). The volume of this confocally obtained optical biopsy is controlled by the aperture at which the light is projected and the size of the pinhole. However, in the living tissue the safe energy density is restricted. Therefore the volume of this in vivo sample must contain ten to hundred of $\mu \mathrm{m}^{3}$ in order to obtain a reliable Raman signal ${ }^{26}$.

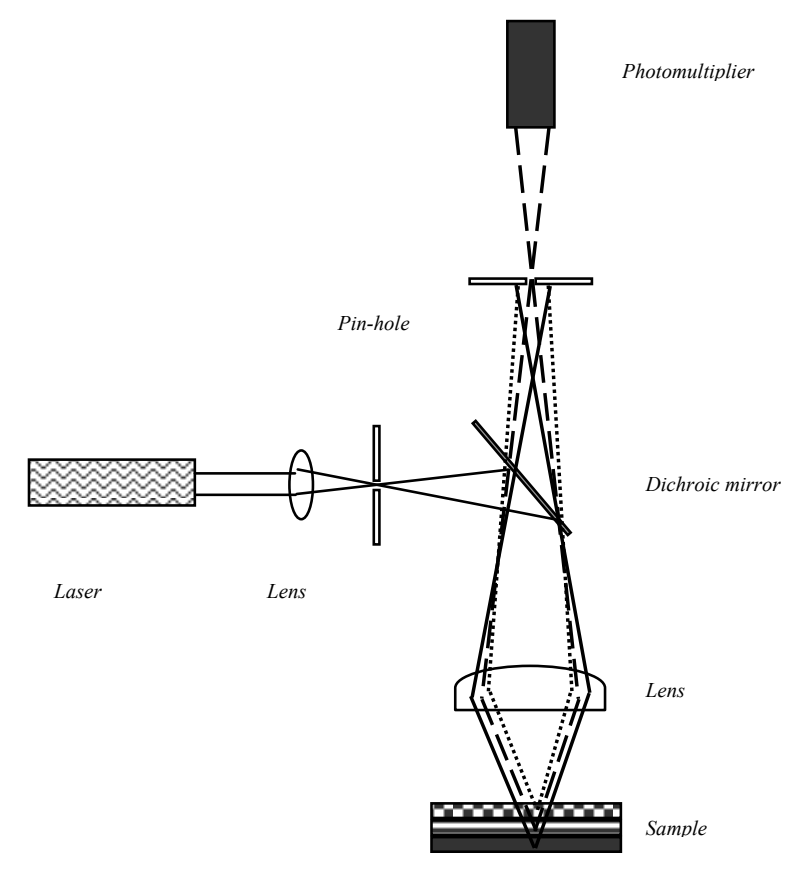

Figure 3. Schematic of a confocal setup. The 'Out of focus' rays are blocked by the pinhole.

In OCT, optical biopsy is achieved by cross-correlation of the coherent scattered light by a reference beam with a known optical length in a low-coherence interferometer. When we need morphological information of the eye, we may use OCT. The spatial resolution of the OCT image is proportional to the bandwidth of the impinging light. A wide bandwidth means a short coherence length and therefore a high spatial resolution. OCT uses near infrared light $(\lambda=0.8 \mu \mathrm{m}$ to $1.5 \mu \mathrm{m})$ with bandwidths from about 20 to 200 $\mu \mathrm{m}$ providing a spatial resolution of $15 \mu \mathrm{m}$ to $1 \mu \mathrm{m}$. The current time domain OCT systems operate at a speed of $200 \mu \mathrm{m} / \mathrm{ms}$. A scatter/intensity scan of the corneal thickness takes about $2 \mathrm{~ms}$. In general the spatial resolution obtained in living samples is much higher when OCT is used compared to those acquired with Raman spectroscopy. 
OCT can be employed to look at the spatial distribution and/or change of the hydration within the cornea in real-time ${ }^{27,28}$ and thus, it can acquire data about the water gradient across this ocular structure. This means that any water quantity change (transport) between the different layers within the cornea can be captured at a time. But if one is interested in the absolute value of the water mass in a specific depth within the cornea, then Raman spectroscopy can be employed to measure this value at a certain depth within the tissue. However, confocal Raman cannot capture the same information at a different depth of the cornea within the same time frame and needs to be refocused and therefore it loses the 'real-time' advantage compared with the $\mathrm{OCT}^{29}$.

\section{Raman spectroscopy}

Raman spectroscopy is an analytical technique for the identification of molecules in gases, liquids and solids by scattering of light. The introduction of lasers in the late 1960's and the implementation of highly sensitive detectors such as CCD cameras and photon counters have launched the practical applications of this mode of spectroscopy. Although Smekal ${ }^{30}$ predicted Raman effect on theoretical grounds in 1923, it was the Indian physicist, C.V. Raman ${ }^{31}$ in 1928 who first discovered it and consequently received the Nobel Prize for his discovery.

Raman spectroscopy is based on inelastic backscattering of light from the sample of interest. The Raman effect comprises a very small fraction, of the scattered photons. In a light scattering experiment, light of a known frequency is scattered from a sample in all directions. The scattered radiation produced by the Raman effect contains information about the energies of molecular vibrations and rotations, and these depend on the particular atoms or ions that comprise the molecule, the chemical bonds connecting them, the symmetry of their molecule structure, and the physical-chemical environment where they reside.

Raman scattered light is frequency-shifted (expressed in $\mathrm{cm}^{-1}$ ) with respect to the excitation frequency, but the magnitude of the shift is independent of the excitation frequency. This "Raman shift" is therefore an intrinsic property of the sample, which can serve as a "finger print" to identify the molecular structure of a given sample. 


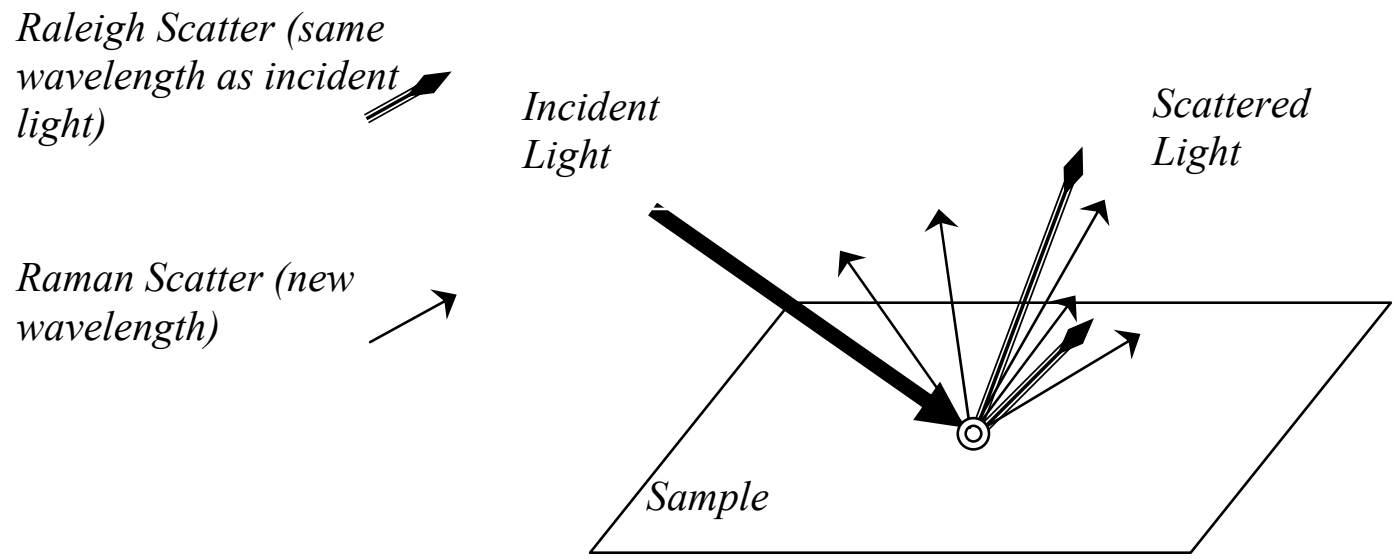

Figure 4. Elastic and inelastic scattered light on a sample.

Because Raman scattering is a weak process compared to Rayleigh scattering, a key requirement to obtain Raman spectra is that the spectrometer provides a high rejection of scattered laser light. The energy of the Raman scattered radiation is less than the incident radiation for the Stokes line and the energy of the Raman scattered radiation is more than the incident radiation for the anti-Stokes line. The energy increase or decrease from the excitation is related to the vibrational energy spacing in the ground electronic state of the molecule and therefore the wavenumber of the Stokes and antiStokes lines are a direct measure of the vibrational energies of the molecule. Thus, in Raman scattering, the energies of the incident and scattered photons are different.

In this thesis, Raman spectroscopy was employed to identify different drugs within the anterior segment of the eye. Raman spectra of these drugs were not reported in the literature and hence they had to be determined by the author. Figure 5 shows specific Raman signals of three different antibiotic, antiviral and antifungal drugs. 


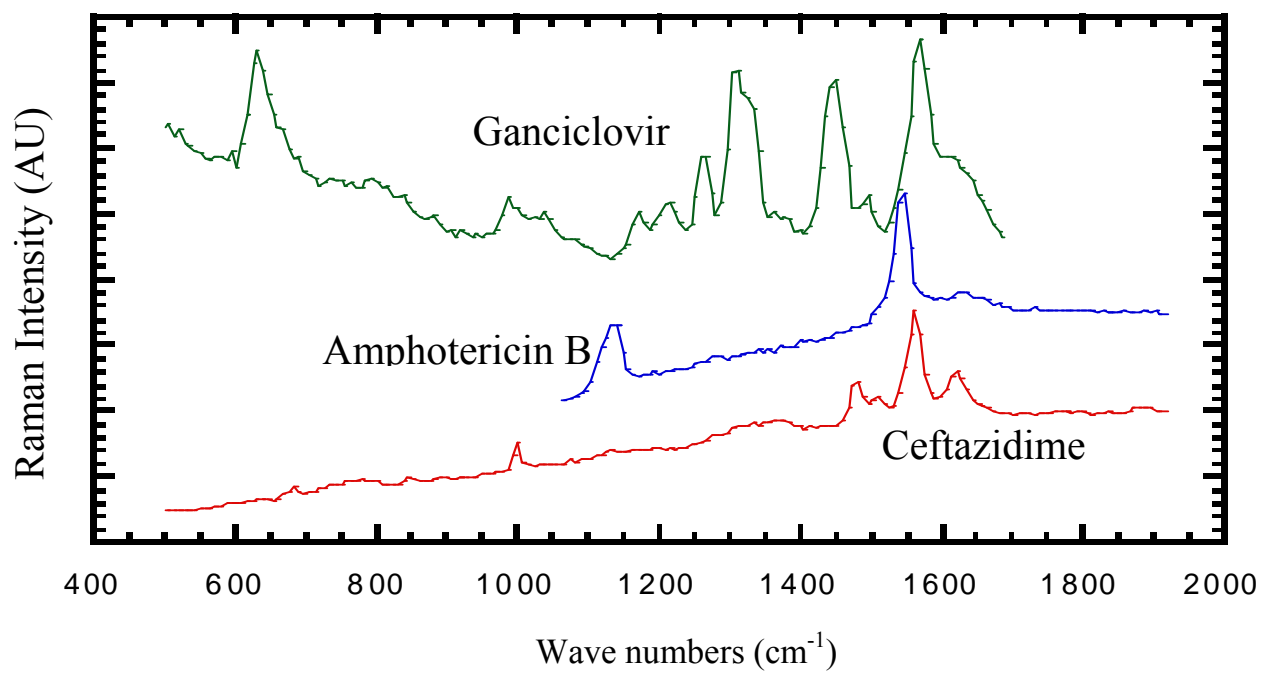

Figure 5. Raman spectra of Ceftazidime, Amphotericine B and Ganciclovir in arbitrary units. The spectra are offset for visualisation purposes.

The linearity between the intensity of the Raman signals for the above shown drugs and their respective concentrations had to be determined as well. Figure 6 exhibits this relation for these compounds.

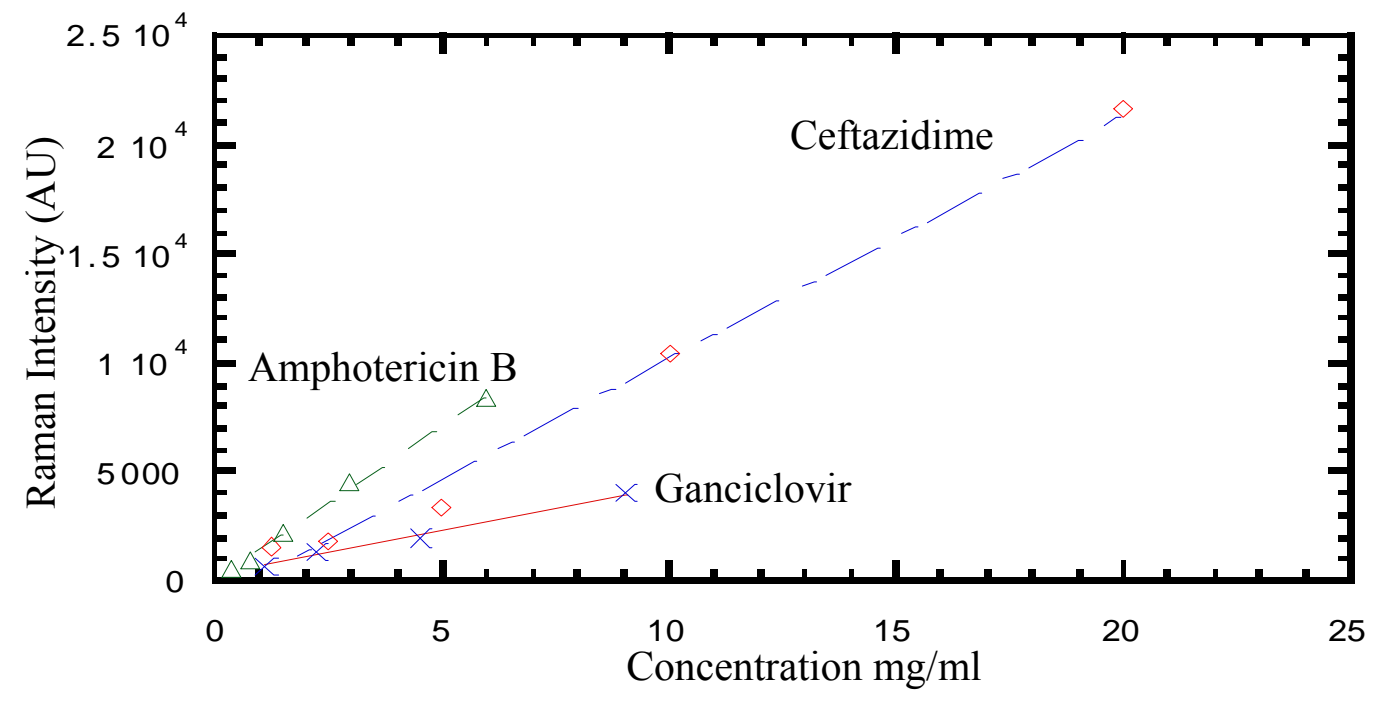

Figure 6. Raman intensity versus different concentrations of Ceftazidime, Amphotericin B and Ganciclovir in quartz cuvettes assessed in an experimental setup. 


\section{General applications of Raman spectroscopy}

Raman spectroscopy has found many applications throughout different industry and science fields such as oil industries, chemical industries, microbiology and remote chemical sensing. Molecular Raman spectroscopy is well suited for the identification of inorganic $^{32}$ and organic pigments ${ }^{33}$, as well as binding media and varnishes ${ }^{34}$. Biology and Medicine have also benefited from this technique for the study of whole organs, tissues and cells ${ }^{35,36}$. Among the first planetary applications were Raman spectroscopic studies of lunar materials brought back by the Apollo missions. There is even a Mars Microbeam Raman Spectrometer (MMRS) planned for operation in anticipation of a landed Mars mission in 2009.

\section{Applications of Raman spectroscopy in ophthalmology}

Investigators from the universities of Maastricht and Galveston have developed a special confocal setup, particularly designed for ophthalmic applications in the early $1990 \mathrm{~s}^{8,29}$. This setup was improved throughout the years and also used in recent years for the Raman studies presented in this thesis. Chapters 2, 3 and 4 exhibit schematic drawings and explain some of the technical details about this setup. Below is an actual picture of this confocal Raman setup for ophthalmic applications.

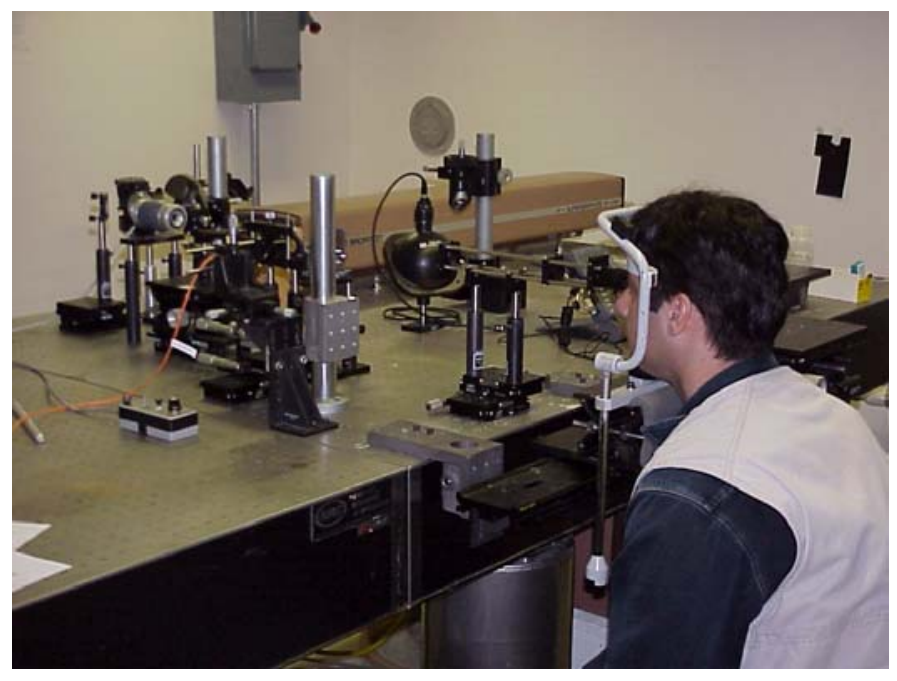

Figure 7. The Raman setup used by the author. 
A particular advantage of Raman spectroscopy is that is can be used to measure spectra in aqueous solutions, which makes it possible to observe living samples and follows their dynamics. This property has been utilized throughout the Raman studies presented in this thesis where the anterior chamber of the eye filled with the aqueous humor was investigated for the presence of drugs and biomaterials in this medium.

\section{Optical coherence tomography}

The first operational OCT was developed by Fujimoto ${ }^{37}$ and coworkers in 1991. OCT is a non-destructive imaging technique, which uses infrared light to visualize subsurface structure in biological tissues. It performs high resolution cross sectional tomographic imaging of the internal microstructure in material and biological systems. The foundation of OCT lies in the early effort on white-light interferometry that led to the progress of optical coherence-domain reflectometry (OCDR), a one-dimensional optical ranging technique ${ }^{38,39}$. OCDR exploits short coherence length light and interferometric detection techniques to attain high sensitivity, high-resolution range information. OCDR was developed for detection of imperfections in fiber-optic lines and network components. Soon after, its potential for ranging measurements in the retina and other eye structures was realized ${ }^{40,41}$.

OCT could be compared to ultrasound imaging, but the magnitude of reflected light is measured instead of reflected sound waves. Analogous to ultrasound, OCT images uses "echoes of light", with a resolution and order of magnitude better than ultrasound and typically around $10-20 \mu \mathrm{m}$ for the conventional OCT. This resolution can be outpaced by an ultra high resolution OCT system equipped with an ultra short pulsed Ti:sapphire laser as its light source, which enables a resolution of almost 1 micrometer ${ }^{42}$.

The axial resolution in low coherence interferometry is related to the bandwidth of the light source according to $\lambda^{2} / \Delta \lambda$. OCT's sectioning capability, determined by the coherence properties of the light source, permits imaging of microscopic tissue structures at depths beyond the reach of conventional bright-field and confocal microscopes. This imaging modality is capable of high-resolution in-vivo measurements of tissue down to a depth of a few millimeters, enabling a true optical biopsy modality. In OCT, the echo time delay and intensity of backscattered or back-reflected light is measured from the internal microstructure in materials or tissues. Scanning a light beam across the tissue produces a cross-sectional image, while a computer records the axial reflectance profiles at each transverse position. The result is a two- or three-dimensional representation of the optical backscattering of the tissue's cross-section, which displays as a gray-scale or false-color image. Thus, OCT images are maps of the reflectivity of a sample. Reflections are generated at the interface of areas of differing indices of refraction. In most tissues, main sources of reflection are collagen fiber bundles, cell walls, and cell nuclei. Dark areas on the image represent homogenous material with low reflectivity, such as air or clear fluids. 


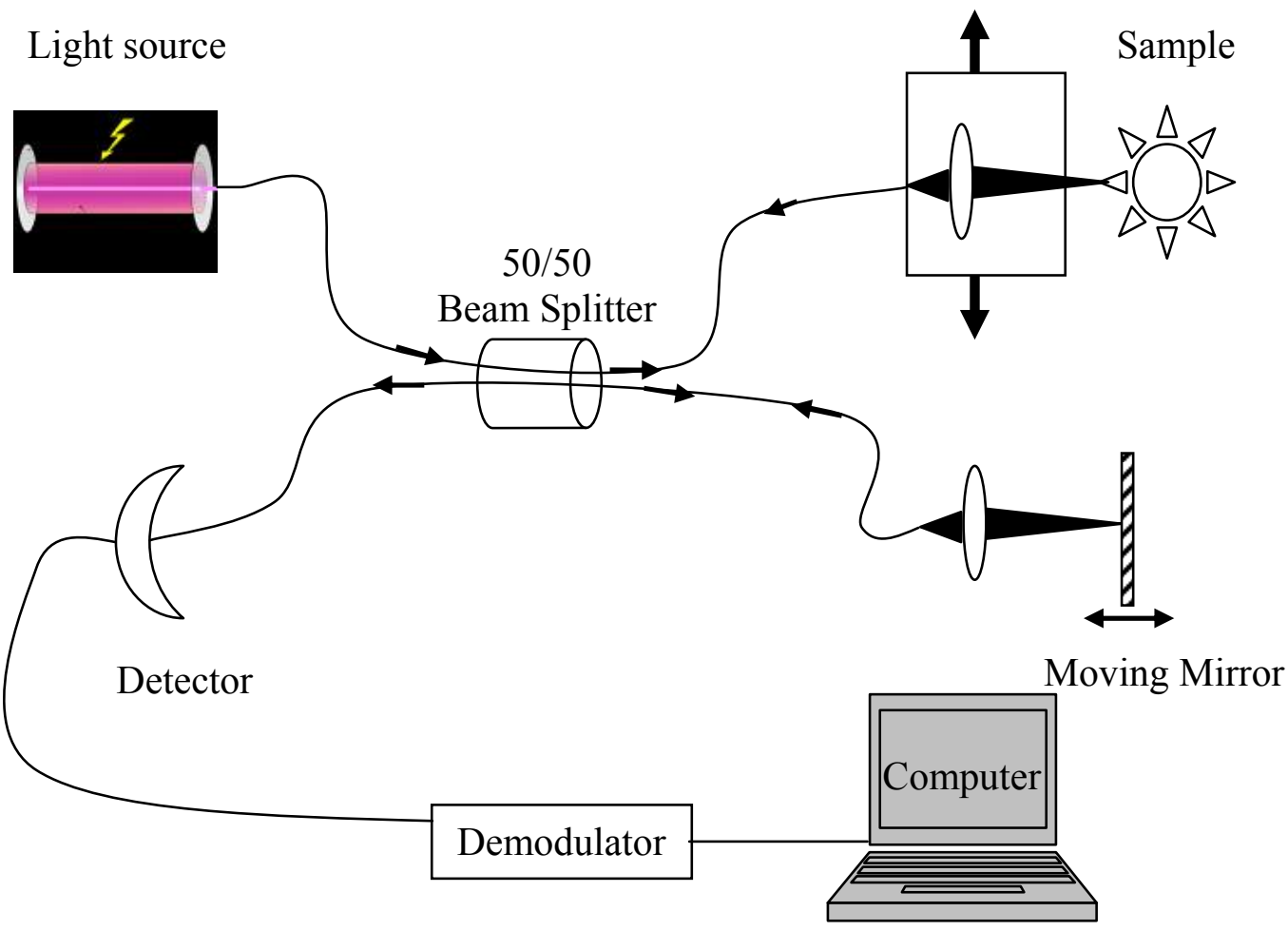

Figure 8. Schematic of an Optical Coherence Tomography system

\section{Applications of OCT in ophthalmology}

The eye with its clear media forms an ideal candidate for this relative new technology. As a result, the most successful clinical application of OCT to date is in retinal imaging. Many studies have been performed to investigate the feasibility of using OCT for the diagnosis and monitoring of posterior segment diseases such as glaucoma, macular edema, macular hole, central serous chorioretinopathy, age-related macular degeneration, epiretinal membranes, optic disc pits, and choroidal tumors while new ocular applications are being reported every year ${ }^{43-45}$.

The in this thesis described research is restricted to the anterior segment of the eye in order to make the confocal Raman data comparable with the OCT data. Chapters 5 to 8 explain some of the technical details and specifications of the OCT used in this thesis. 


\section{Axial scatter intensity mode with OCT}

Typically, an OCT system is used to furnish in-depth images of a sample crosswise. Another competent property of an OCT is its ability to detect changes in tissues like the physiologically caused alterations in tissue thickness and assess optical properties related to the hydration level. This is realized in this thesis by connecting a digital oscilloscope to an OCT system. This makes qualitative and quantitative studying of the interferential pulses much more practical. For instance, while probing the cornea, the backreflection of the light from different interfaces of the cornea (cornea/air and cornea/aqueous humor) provides high pulses resulting from the change of the refractive indices of the involved media. The time delay between these pulses provides the means to calculate the optical thickness of the sample of interest (in this thesis the cornea). This means using the refractive index of the cornea as reported close to 1.3758 by other investigators ${ }^{46,47}$, one can calculate and follow the geometric thickness change of the cornea temporally during and/or after being subjected to any external stress (i.e. dehydration in air, use of topical drugs). This makes monitoring of the effects of any external stimuli on the cornea and the resulted impacts on its physiology and biochemistry possible. Moreover, this monitoring can happen in a real-time and continuo fashion since probing the eye is completely non-contact, eye safe, fast and painless.

Aside from the backreflection data acquisition, the backscattering of the stromal part of the cornea can also be assessed and evaluated. This provides information about any possible change within the structure of hydration gradient inside the cornea and its dynamics under influence of applied drug and/or stress. 
I: Imaging mode (B-scan)

II: scatter intensity mode (A-scan)
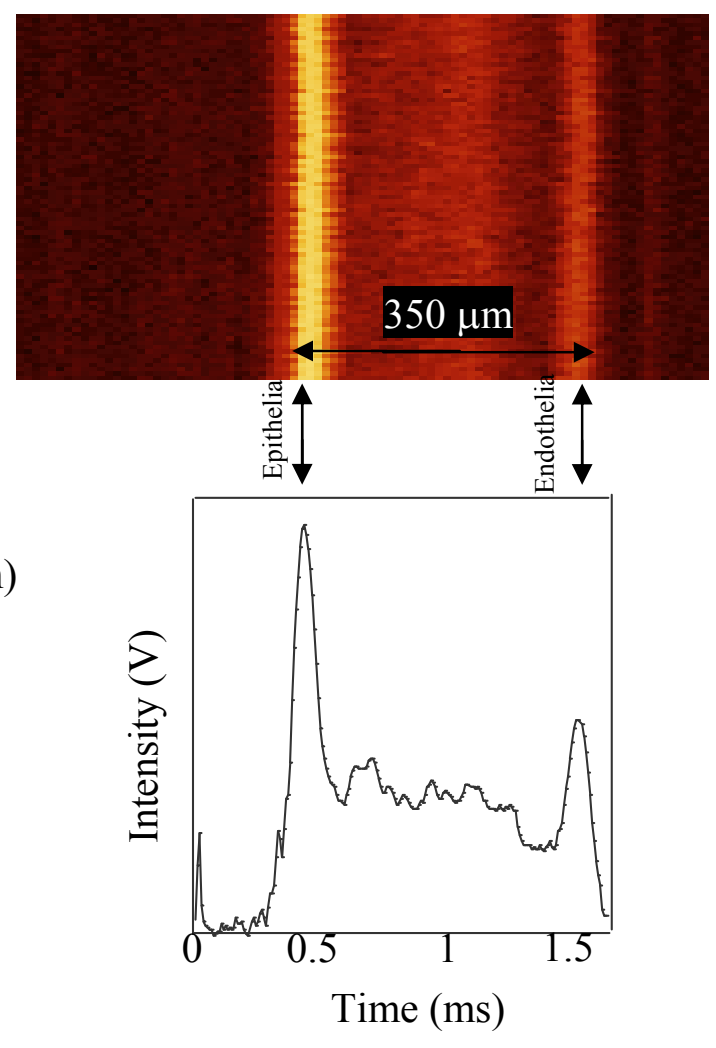

Figures $9 A$ and $9 B$. Typical image of a rabbit cornea while the probing beam of the OCT was focused on its epithelial surface (the bright yellow line). The oscilloscopic plot below this image shows the interferential peaks of the same image as visualized by a digital oscilloscope for a more quantitative assessment.

Other investigators have explored capability of OCT in the measurement of other substances. The feasibility of OCT in the measurement of blood glucose based on studying of the tissue scattering change has been shown in a pilot study in human ${ }^{48}$. It has also been shown that other body solutes can change the refractive index mismatch between the interstitial fluid (ISF) and scattering centers in tissue ${ }^{49}$. A more recent publication also demonstrated the capability of OCT for depth-resolved monitoring and quantifying of glucose diffusion in the sclera of the eye $\mathrm{e}^{50}$.

A commercial OCT system is mainly used by clinicians in an imaging (2D) mode for retinal imaging, quantification of the thickness of nerve fiber layers as well as highresolution imaging of the anterior chamber of the eye. However, in this study an A-scan mode has been used to measure the physiology of the perturbed cornea (change of hydration and monitoring the water movement) based on its backscatter changes in time and space. 
During the OCT related ophthalmic investigations performed in this thesis, a fixation device was developed and used to minimize the eye movements (e.g. from respiration) with respect to the probing beam. All animal studies conducted for this thesis were performed in adherence to the ARVO statement for the use of animals in ophthalmic and vision research.

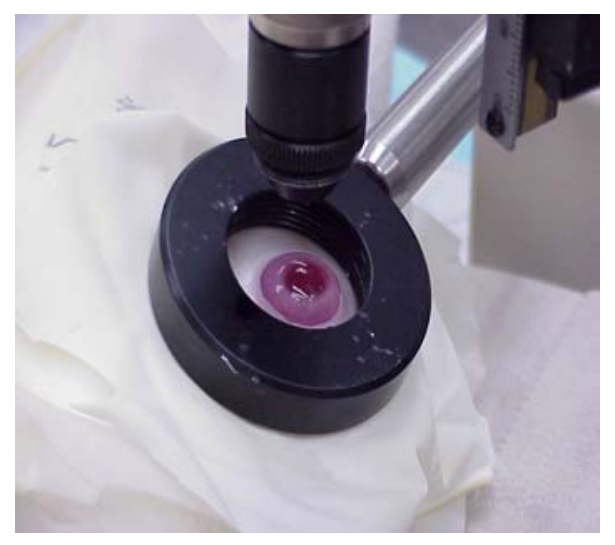

Figure 10. The probe of the OCT device used in this thesis while aimed on the rabbit eye in vivo.A self-manufactured rubber ring attached to an outer metal ring was used to stabilize the eye. 


\section{Aim of thesis}

It is well known that many changes in anatomy and physiology of the eye go hand in hand and thus the ability to monitor these changes (ideally simultaneously) could enhance the understanding of both function and pathology. Meaningful and objective monitoring of these changes may happen if the changes can be quantified with a reasonable precision without invasive perturbation. The aim of this thesis was to investigate the potential applications of two different optical techniques to look at these changes in the anterior segment of the eye. As argued in the introduction, each technique can traditionally either monitor the morphology and thus anatomy (OCT), or the chemical/analytical aspect of the substances and thus the forthcoming physiology (Raman) in the probed area. This thesis suggests that OCT may also enable some quantification for analytical sensing of a well-known substance while undergoing a quantity change. Furthermore, this work opens the question for a combination diagnostic device. Different combinations of OCT with fundus imaging technologies have already been introduced to offer the best of both worlds for retinal imaging ${ }^{51,52}$. One could argue that a quantitative imaging system suitable for routine in vivo assessment of the ocular microstructure would make it possible to correlate structural changes with analytical changes. Therefore, combining Raman spectroscopy with OCT as a combination diagnostic device could potentially offer a useful monitoring system that provides both structural and analytical information from the same area in real-time. 


\section{References}

1. Taylor HR, McCarty CA, Nanjan MB. Vision impairment predicts five-year mortality. Trans Am Ophthalmol Soc. 2000;98:91-96.

2. Weih LM, Hassell JB, Keeffe JE. Assessment of the impact of vision impairment. Invest Ophthalmol Vis Sci. 2002;43:927-935.

3. Wang JJ, Mitchell P, Smith W, et al. Incidence of nursing home placement in a defined community. Med J Aust. 2001;174:271-275.

4. Wang JJ, Mitchell P, Smith W, et al. Factors associated with use of community support services in an older Australian population. Aust NZ J Public Health. 1999;23:147-153.

5. Haymes SA, Leblanc RP, Nicolela MT, Chiasson LA, Chauhan BC. Risk of falls and motor vehicle collisions in glaucoma. Invest Ophthalmol Vis Sci. 2007 Mar;48(3):1149-55.

6. McCarty CA, Fu CL, Taylor HR. Predictors of falls in the Melbourne Visual Impairment Project. Aust NZ J Public Health. 2002;26:11611-11619.

7. Rovner BW, Casten RJ, Tasman WS. Effect of depression on vision function in age-related macular degeneration. Arch Ophthalmol. 2002;120:1041-1044.

8. Erckens RJ, March WF, Jongsma FH, Wicksted JP, Hendrikse F, Smit EE, Motamedi M. Noninvasive Raman spectroscopic identification of intraocular lens material in the living human eye. J Cataract Refract Surg. 2001 Jul;27(7):1065-70.

9. Erckens RJ, Hosseini K, March WF, Jongsma FH, Wicksted JP, Li K, Hendrikse F, Raman spectroscopy: noninvasive determination of silicone oil in the eye: potential applications for intraocular determination of biomaterials. Retina. 2002 Dec;22(6):796-9.

10. Smit EE, Erckens RJ, Hendrikse F, Motamedi M, Wicksted JP, March WF. Identification of intraocular lens materials using confocal Raman spectroscopy. J Cataract Refract Surg. 1999 Nov;25(11):1498-504.

11. Bernstein PS, Zhao DY, Sharifzadeh M, Ermakov IV, Gellermann W. “ Resonance Raman measurement of macular carotenoids in living human eye". Arch Biochem Biophys. 2004 Oct 15;430(2):163-9.

12. Berman E.R. Biochemistry of the Eye, 1991 Plenum Press, New York, 1991.

13. Kinsey V.E. "Ion movement in the eye". Circulation, 21, 968-987 (1960). 
14. Mao Y, Wu Z, Lin S, Li W. Measurement and the clinical significance for the intraocular concentrations of carboplatin after systemic and local administration in rabbits. Scholar paper of Ophthalmol. 2004 Jul;20(2):123-6.

15. Dias C, Nashed Y, Atluri H, Mitra A. Ocular penetration of acyclovir and its peptide prodrugs valacyclovir and val-valacyclovir following systemic administration in rabbits: An evaluation using ocular microdialysis and LC-MS. Curr Eye Res. 2002 Oct;25(4):243-52.

16. Moseley H, Foulds WS, Allan D, Kyle PM, Routes of clearance of radioactive water from the rabbit vitreous. Br J Ophthalmol 1984 Mar ;68(3):145-51.

17. Welge-Lussen L, Schimmel KP. Examinations of aminoacids in aqueous humour after intravitreous injection of ascorbic acid. Klin Monatsbl Augenheilkd 1978;172(6):824-30.

18. Hollander DA, Aldave AJ. Drug-induced corneal complications. Curr Opin Ophthalmol. 2004 Dec;15(6):541-8.

19. Kalra L, Bone MF. The effect of nebulized bronchodilator therapy on intraocular pressures in patients with glaucoma. Chest 1988;93:739-741.

20. Davis JL, Taskintuna I, Freeman WR, et al. Iritis and hypotony after treatment with intravenous cidofovir for cytomegalovirus retinitis. Arch Ophthalmol 1997;115:733-737.

21. Maurice DM. The transparency of the corneal stroma. Vision Res. 1970 Jan;10(1):107-8.

22. Jaffe GJ, McCallum RM, Branchaud B, Skalak C, Butuner Z, Ashton P. Longterm follow-up results of a pilot trial of a fluocinolone acetonide implant to treat posterior uveitis. Ophthalmology. 2005 Jul;112(7):1192-8.

23. Kamal A, King A, Sylvestri G, Dhillon B. Sustained release intravitreal ganciclovir implant as salvage treatment in AIDS related cytomegalovirus retinitis. Br J Ophthalmol. 1998 Mar;82(3):333.

24. Hiratani H, Fujiwara A, Tamiya Y, Mizutani Y, Alvarez-Lorenzo C. Ocular release of timolol from molecularly imprinted soft contact lenses. Biomaterials. 2005 Apr;26(11):1293-8.

25. Eljarrat-Binstock E, Raiskup F, Frucht-Pery J, Domb AJ. Transcorneal and transscleral iontophoresis of dexamethasone phosphate using drug loaded hydrogel. J Control Release. 2005 Sep 2;106(3):386-90.

26. Minsky M. Memoir on inventing the confocal scanning microscope. Scanning 1988;10:128-38. 
27. Kholodnykh, A.I., Hosseini, K., Larin, K.V., Vassilieva, A.A., Esenaliev, R.O., Motamedi, M. Accurate in vivo measurements of the corneal light backscattering in-depth distribution with optical coherence tomography for different hydration states. Proceedings of SPIE, Volume 5316, July 2004, pp. 12-15

28. Hosseini K, Kholodnykh AI, Petrova IY, Esenaliev RO, Hendrikse F, Motamedi $\mathrm{M}$. Monitoring of rabbit cornea response to dehydration stress by optical coherence tomography. Invest Ophthalmol Vis Sci. 2004 Aug;45(8):2555-62.

29. Bauer NJ, Wicksted JP, Jongsma FH, March WF, Hendrikse F, Motamedi M. Noninvasive assessment of the hydration gradient across the cornea using confocal Raman spectroscopy. Invest Ophthalmol Vis Sci. 1998 Apr;39(5):831-5.

30. Smekal, A. (1923) Naturwiss. 11, 873.

31. Raman, C.V., Krishnan, K.S. (1928), Nature 121, 501.

32. B. Wehling, P. Vandenabeele, L. Moens, R. Klockenkämper, A. von Bohlen, G. Van Hooydonk and M. De Reu, Mikrochim. Acta 130, 253-260 (1999).

33. P. Vandenabeele, L. Moens, H.G.M. Edwards and R. Dams, A Raman Spectroscopic Database of Azo-pigments and application to Modern Art Studies, J. Raman Spectr. 2000.

34. P. Vandenabeele, B. Wehling, L. Moens, H. Edwards, M. De Reu and G. Van Hooydonk, Anal. Chim. Acta 407, 261-274 (2000).

35. Puppels GJ. Confocal Raman Spectroscopy: a new look at cells and chromosomes. Thesis. University Twente, The Netherlands. 1991.

36. Ozaki Y. Medical Application of Raman Spectroscopy. Applied Spectroscopy Reviews 1988; 24(3\&4): pp. 259-312.

37. Huang D, Swanson EA, Lin CP, Schuman JS, Stinson WG, Chang W, Hee MR, Flotte T, Gregory K, Puliafito CA, and J.G. Fujimoto. Optical coherence tomography. Science. 1991 Nov 22;254(5035):1178-81.

38. Youngquist R.C., Carr S. and Davies D.E.N. Optical coherence-domain reflectometry: A new optical evaluation technique. Opt. Lett., 12:158-160, 1987.

39. Takada K, Yokohama I, Chida K, and Noda J. New measurement system for fault location in optical waveguide devices based on an interferometric technique. Appl. Opt., 26:1603-1606, 1987.

40. Fercher A.F., Mengedoht K., and Werner W. Eye-length measurement by interferometry with partially coherent-light. Opt. Lett., 13:186-188,1988. 
41. Hitzenberger C.K, Optical measurement of the axial eye length by laser Doppler interferometry. Inv. Ophthalmol. Vis. Sci.,32:616-624, 1991.

42. Morgner U. Kärtner F. X, Cho S.H, Chen Y, Haus H.A, Fujimoto J.G, Ippen E.P, Scheuer V, Angelow G, and Tschudi T. Sub-two-cycle pulses from a Kerr-lens mode-locked Ti:Sapphire laser. Opt. Lett.,24:411-413, 1999.

43. Bijisma WR, Stilma JS. Optical coherence tomography, an important new tool in the investigation of the retina. Ned Tijdschr Geneeskd. 2005 Aug 20;149(34):1884-91.

44. Olmedo M, Cadarso-Suarez C, Gomez-Ulla F, Val C, Fernandez I. Reproducibility of optic nerve head measurements obtained by optical coherence tomography. Eur J Ophthalmol. 2005 Jul-Aug;15(4):486-92.

45. Ko TH, Fujimoto JG, Schuman JS, Paunescu LA, Kowalevicz AM, Hartl I, Drexler W, Wollstein G, Ishikawa H, Duker JS. Comparison of ultrahigh- and standard-resolution optical coherence tomography for imaging macular pathology. Ophthalmology. 2005 Nov;112(11):1922. Epub 2005 Sep 23.

46. Aubert H, Matthiesen L. Graefe-Saemisch Handbuch der gesammten Augenheilkunde. 1st ed. Leipzig, Germany: Engelmann; 1876:409.

47. Maurice DM. The structure and transparency of the cornea. J Physiol. 1957;136:263-286.

48. Larin KV, Eledrisi MS, Motamedi M, Esenaliev RO. Noninvasive blood glucose monitoring with optical coherence tomography: a pilot study in human subjects. Diabetes Care. 2002 Dec;25(12):2263-7.

49. Larin KV, Motamedi M, Ashitkov TV, Esenaliev RO. Specificity of noninvasive blood glucose sensing using optical coherence tomography technique: a pilot study. Phys Med Biol. 2003 May 21;48(10):1371-90.

50. Ghosn MG, Tuchin VV, Larin KV. Depth-resolved monitoring of glucose diffusion in tissues by using optical coherence tomography. Opt Lett. 2006 Aug 1;31(15):2314-6.

51. Rosen RB, Hthaway M, Rogers J, Pedro J, Garcia P, Dobre GM, Weitz R, Podoleanu A. Simultaneous OCT/SLO/ICG system. Progr. biomed. opt. Imaging. 2006, vol. 7, no2.

52. Kocaoglu OP, Uhlhorn SR, Hernandez E, Juarez RA, Will R, Parel JM, Manns F. Simultaneous Fundus Imaging and Optical Coherence Tomography of the Mouse Retina. Invest Ophthalmol Vis Sci. 2007 Mar;48(3):1283-89. 


\section{Chapter 2}

\section{Noninvasive Detection of Ganciclovir in Ocular Tissue by Raman Spectroscopy: Implication for Monitoring of Drug Release}

JOURNAL OF OCULAR PHARMACOLOGY AND THERAPEUTICS

Volume 18, Number 3, 2002 C Mary Ann Liebert, Inc.

K. HOSSEINI ${ }^{1,2}$, W. MARCH ${ }^{1}$, F.H.M. JONGSMA ${ }^{2}$, F. HENDRIKSE ${ }^{2}$ and M. MOTAMEDI ${ }^{1}$

The University of Texas Medical Branch, Department of Ophthalmology and Visual Sciences,

Galveston, Texas

2 Department of Ophthalmology, AZM, Maastricht, The Netherlands 


\begin{abstract}
Introduction: Treatment of various pathological conditions in ophthalmology, such as cytomegalovirus (CMV) retinopathy and endophthalmitis, requires a local drug intervention rather than a systemic approach. Accurate knowledge of intraocular drug concentration can permit the ophthalmologist to maintain drug levels within the therapeutic levels necessary for an optimal

prognosis, while preventing or minimizing toxicity associated with drug overdose. Purpose: To develop a noninvasive/noncontact method for quantification of the local concentration of ganciclovir in the ocular media.

Methods: An integrated CCD based Raman spectroscopic system designed specifically for ophthalmic applications was used to noninvasively detect the presence of ganciclovir in the ocular media. Various known concentrations of ganciclovir were injected into the aqueous humor of rabbit eyes in a pilot study, in vivo. Raman spectra were then acquired by focusing an argon laser beam within the anterior chamber of the eye. The specific Raman signature of ganciclovir was assessed at several concentrations.

Results: Spectral features unique to ganciclovir were identified and distinguished from those of ocular tissue. The amplitudes of the spectral peaks corresponding to ganciclovir exhibited a linear dependence on the local concentration of the drug in the anterior chamber of the eye. Conclusion Raman spectroscopy may offer an effective tool for the noninvasive assessment of the local concentration of ganciclovir in the ocular media. This technique offers the potential to determine both the amount and the rate of the drug release from implants designed to deliver antiviral drugs locally within the eye. The availability of such data could enable the ophthalmologist to improve treatment efficacy by avoiding premature or late surgical replacement of the implants.
\end{abstract}




\section{Introduction}

Clinical studies have demonstrated the potential utilities of local drug delivery in ocular media via direct injection or surgical placement of drug releasing implants ${ }^{1,2}$. The safety and efficacy of these techniques are highly dependent on the drug dose and the rate of drug release. Ganciclovir sustained release intravitreal implants are available for treatment of CMV retinitis. Several other implants are also currently under investigation. Increasingly novel technologies, such as intravitreal microdialysis ${ }^{3}$, are under investigation to deliver drugs within the eye. There currently exists no method to gauge the function of an implant at any time after implantation other than a tap of the aqueous or vitreous humor. It would be helpful to have a noninvasive means of monitoring drug release to accurately gauge the implant's function and viable lifetime, which currently can only be estimated.

The acquisitions of noninvasively obtained feedback data indicating the presence and concentration of a drug in the ocular media can help determine the most effective timing for (re) implantation. Continuous monitoring of drug release could allow for correcting the patient to patient variation in the kinetics of drug release from ganciclovir implants. Currently, the schedule of additional implants is based on clinical evaluation. However, a noninvasive means of monitoring drug release would allow clinicians to optimize the timing of implantation. In some cases, a lower frequency in implant exchange may even be achieved. Martin et al. concluded that multiple reentries through a single wound might be associated with an increased risk for vitreous hemorrhage ${ }^{4}$. In another study, Lim et al. found a $12 \%$ complication rate in the ganciclovir implant procedures 5 .

Noninvasive monitoring of this particular drug is not only beneficial in CMVretinitis, but ganciclovir is also under investigation for the treatment of other conditions.

For example, ganciclovir has been utilized as an alternative therapy for anterior uveitis ${ }^{6}$, and there has been a report suggesting the benefit of its use in the form of intravitreal injections as adjunctive therapy in the management of immunocompetent patients with necrotizing herpetic retinopathy ${ }^{7}$.

Raman spectroscopy measures the vibrational frequencies of molecules excited by optical radiation. The Raman spectrum is unique for any particular molecule and it can serve as the molecule's "fingerprint". Theoretically, Raman spectra can be used noninvasively to measure the concentration of a particular chemical in mixtures and solutions ${ }^{8,9}$.

Various studies have shown that when substances are administered as intravitreal injections in the eye, some of the substance appears in the anterior chamber $^{10,11}$. The present study was designed to determine if confocal Raman (Conforam) spectroscopy could be used to assess the concentration of ganciclovir in the anterior chamber in a noninvasive manner.

To our knowledge, no study has been done in the past to investigate the feasibility of a noninvasive approach in detecting ganciclovir in ocular spaces. We have determined Raman signatures for ganciclovir, and noninvasively detected and measured certain concentrations of this drug in the anterior chamber of white New Zealand rabbit eyes. 


\section{Materials and methods}

Figure 1 shows a schematic drawing of an integrated CCD based scanning confocal Raman spectroscopy system with a long working distance for the noninvasive biochemical characterization of ocular tissue. The key components of the system are a single grating spectrometer with a focal length of 0.5 meter and an aperture of F:4 (SPEX500M; spex industries, Edison, NJ) with a nitrogen cooled CCD camera with a high quantum efficiency for rapid signal detection, an argon-ion laser emitting a linearly polarized beam at $514.5 \mathrm{~nm}$. A dielectric filter positioned at $45^{\circ}$ with respect to the laser beam served as a band filter for the incident polarized laser beam. A long working distance microscope objective lens (magnification, X25; numerical aperture 5 0.5; focal length $510 \mathrm{~mm}$; Jena, Karl Zeiss, Oberkochen, Germany) that acts as the focusing device for the incident light and the collecting lens for the Raman backscattered light. This lens permits noninvasive probing of a considerable axial distance in the eye. An optical fiber coupled to the spectrometer collects the Raman backscattered light and acts similarly to a confocal pinhole.

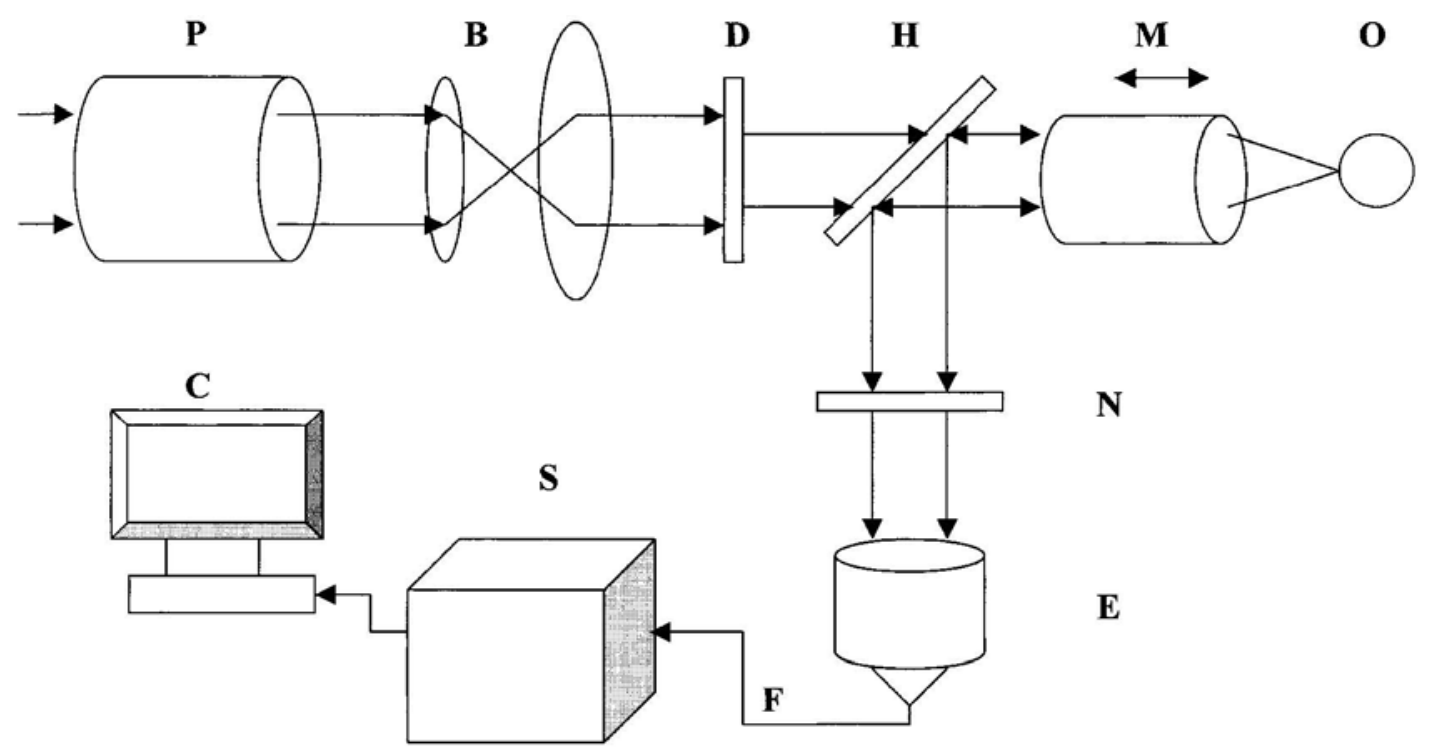

Figure 1. Confocal Raman scattering setup consisting of a polarization rotator $(P)$, beam expander (B), diaphragm (D), band pass filter/Raman scattered light reflector $(H)$, microscope objective $(M)$, eye $(O)$, notch filter $(N)$, exit lens $(E)$, fiber probe $(F)$, spectrometer $(S)$, and computer $(C)$.

The Raman signature of gancilovir was determined using the Conforam spectroscope on samples of Citrovene (Roche) placed in quartz cuvettes as a function of concentration using a laser power of $25 \mathrm{~mW}$ and an acquisition time of one second. To 
enhance the ability of our method to quantify the inherently weak Raman signal of this media, we applied a technique called "binning" to the chip of the CCD camera. Binning improves signal to noise ratio by combining the charge in adjacent pixels and increasing the intensity by an amount proportional to the number of pixels binned. The spectrometer's grating was set to acquire the signals within the range of the range of 600 $\mathrm{cm}^{-1}$ to $1900 \mathrm{~cm}^{-1}$. Subsequently, Raman spectra of the aqueous humor of white New Zealand rabbits were acquired in vivo using the same apparatus.

In this study, the rabbits ( $\mathrm{n}=4,8$ eyes) were anaesthetized using a mixture of xylazine and ketamine injected intramuscularly. All animal care and treatment followed humane laboratory methods, as described in the Guide of Care and Use of Laboratory Animals (NIH Publication \#8623) and peer review approved by the Institutional Animal Care and Use Committee of the University of Texas Medical Branch at Galveston. The animals were then placed and stabilized in front of the laser beam. A lid speculum was placed and probing laser beam was focused in the anterior chamber. Positioning the probing beam is facilitated by the fact that the cornea and crystalline lens produce a strong Raman signal in the region of 2840 $3000 \mathrm{~cm}^{-1}$. The absence of these signals confirmed the position of the probing beam ensuring placement of the beam within the anterior chamber. In these studies, series of scans were made in order to determine the native peaks visible in the anterior the anterior chamber void of any foreign substance. Then a predetermined volume $(0.02-0.12 \mathrm{ml})$ of aqueous humor was removed via a paracentesis using a $30 \mathrm{G}$ needle and replaced with an identical volume containing various concentrations of ganciclovir in order to reach desired levels of the antiviral drug within the aqueous humor. Specific Raman signatures were obtained for the various amounts (100 mg and $600 \mathrm{mg}$ ) of ganciclovir that had been injected in the aqueous humor of rabbit eyes in vivo. Finally, a high dose of ganciclovir $(2000 \mathrm{mg}$ ) was injected through pars plana in order to examine the ability of Raman spectroscopy to detect the presence of the drug in the vitreous body.

\section{Results}

Figure 2 exhibits the Raman signature of gancilovir as determined by the Conforam spectroscope on samples of Citrovene (Roche) placed in quartz cuvettes. The correlation between the intensity of the Raman signal versus different drug concentration drug concentrations is shown in Figure 3. This figure demonstrates the ability of this technique to measure drug concentration because it correlates with the area under the curve in the range of $1426 \mathrm{~cm}^{-1}$ to $1466 \mathrm{~cm}^{-1}$.

Subsequently, Raman spectra of the aqueous humor of white New Zealand rabbits were acquired using the same apparatus. The resulting spectra were compared to the scans of the aqueous prior to the administration of the drug into the anterior chamber (Figure 4a).

As shown in Figure 2 ganciclovir has a broad spectral feature in the range between $600 \mathrm{~cm}^{-1}$ and $1900 \mathrm{~cm}^{-1}$. The Raman spectrum of the anterior chamber without drug shows many small peaks in the same region as well, which to a certain extent would overlap with those of ganciclovir when monitored in the aqueous humor. These various 
spectral peaks in the aqueous humor are due to the vibrational modes of the biological molecules such as lactate, glucose, urea and amino acids that are normally present in this medium. When compared to ocular tissue, ganciclovir demonstrated unique spectral features near 631, 1263, 1302, 1448 and $1560 \mathrm{~cm}^{-1}$. The differences between Raman spectra of tissue and that of ganciclovir became more apparent when these two spectra were subtracted from one another (Fig. 4b). The amplitude of the spectral peak corresponding to ganciclovir was determined to be linearly dependent on the local concentration of ganciclovir in the anterior chamber of the eye.

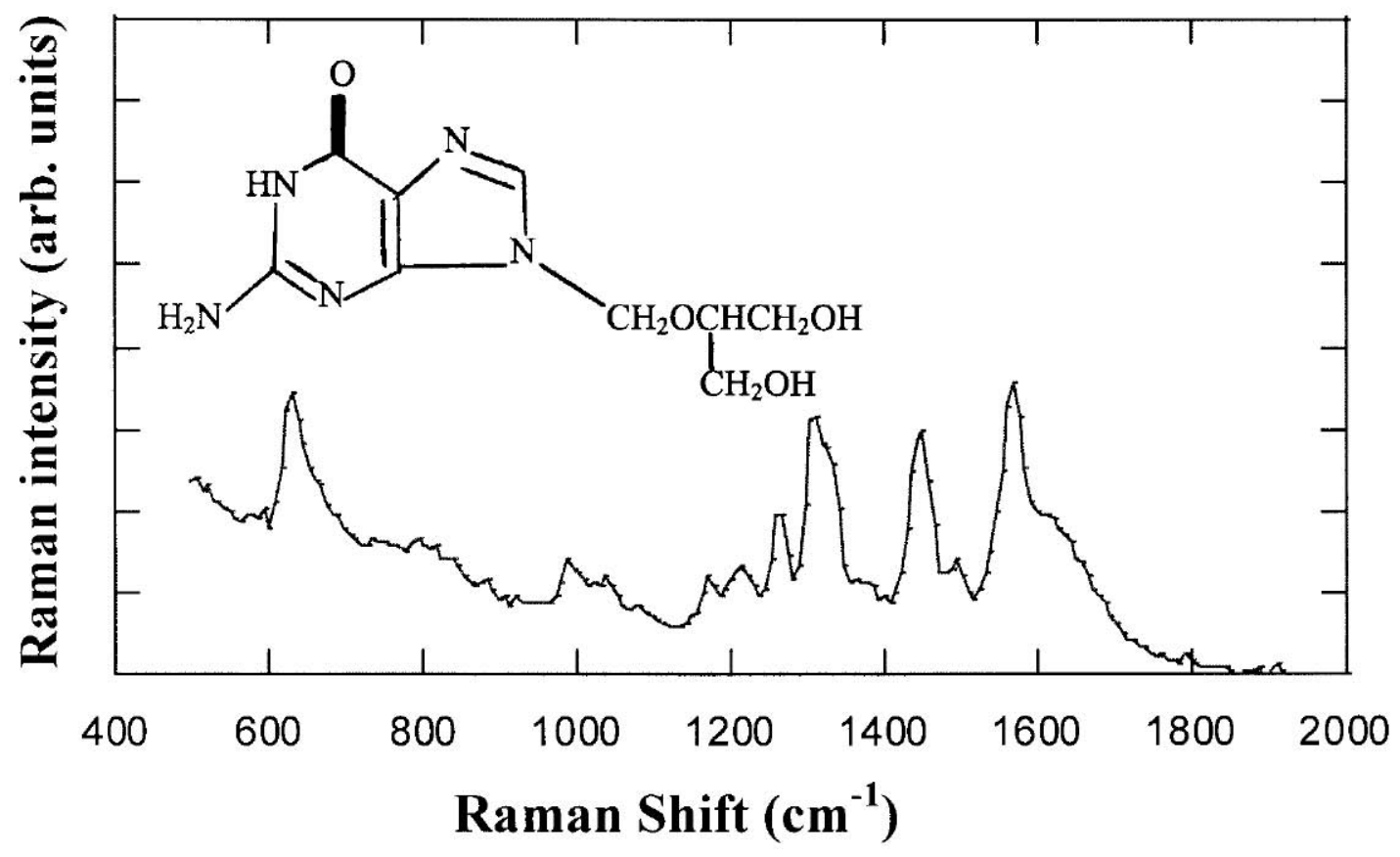

Figure 2. Molecular structure of Ganciclovir along with its Raman Signature 


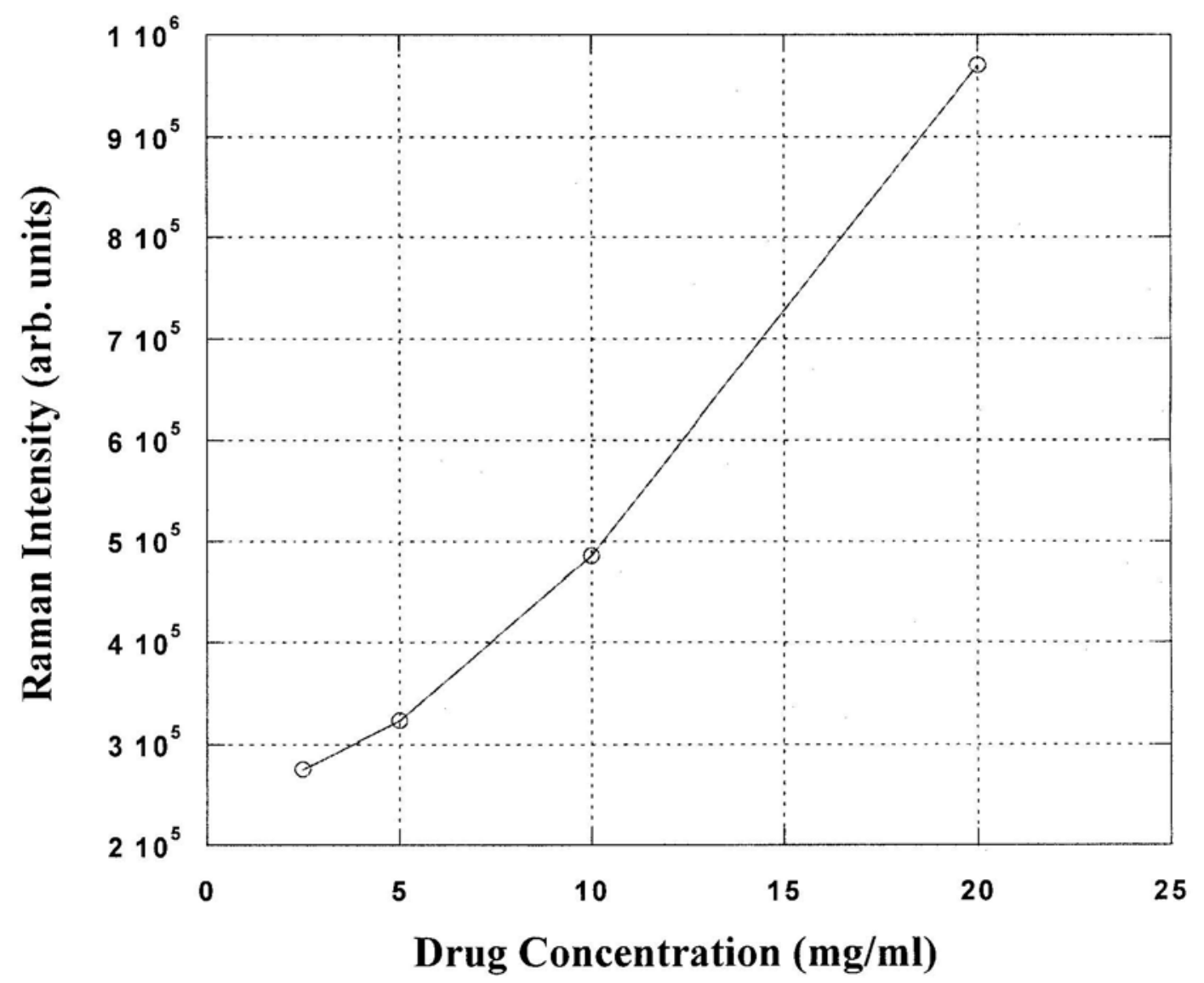

Figure 3. Raman intensity versus different concentrations of ganciclovir (2.5, 5, 10 and $20 \mathrm{mg} / \mathrm{ml}$ ) in quartz cuvettes assessed in an experimental setup

The ability of Raman spectroscopy to assess the concentration of ganciclovir noninvasively in vivo can be appreciated when one considers the results of the measurements performed based on estimating the same area under the curve as it was done in our sample study experiment on all eyes (total of 8 eyes). Statistical analysis of the intensity in those areas for the group with $100 \mathrm{mg}$ revealed an average of 3932.3, and a standard deviation of 1930.4 counts per second, while the average for the group with $600 \mathrm{mg}$ was 18397.0 with a standard deviation of 2409.4 counts per second. The disparity between these numbers shows the dependence of the strength of Raman signal on the concentration of ganciclovir in vivo.

Figure 5 shows a Raman spectrum obtained from the vitreous following intravitreal injection of ganciclovir. The spectrum of ganciclovir has peaks similar to those observed in the anterior chamber showing that specific spectral feature of ganciclovir can be detected within the vitreous chamber of the eye. However, the optical configuration of the current Conforam apparatus are not optimized for monitoring posterior part of the eye so the amount of ganciclovir in vitreous could not be quantified at low concentration of drug. 


\section{Discussion}

Currently, various intravitreal drugs are administered according to therapeutic dosages that are empirically assessed by clinical experiments either with animal models and/or with a limited number of human studies. Noninvasive monitoring of a drug's concentration would be desirable because these concentrations in the ocular media are subjected to unpredictable changes that are influenced by many factors such as absorption, distribution, biotransformation, and elimination of drugs.

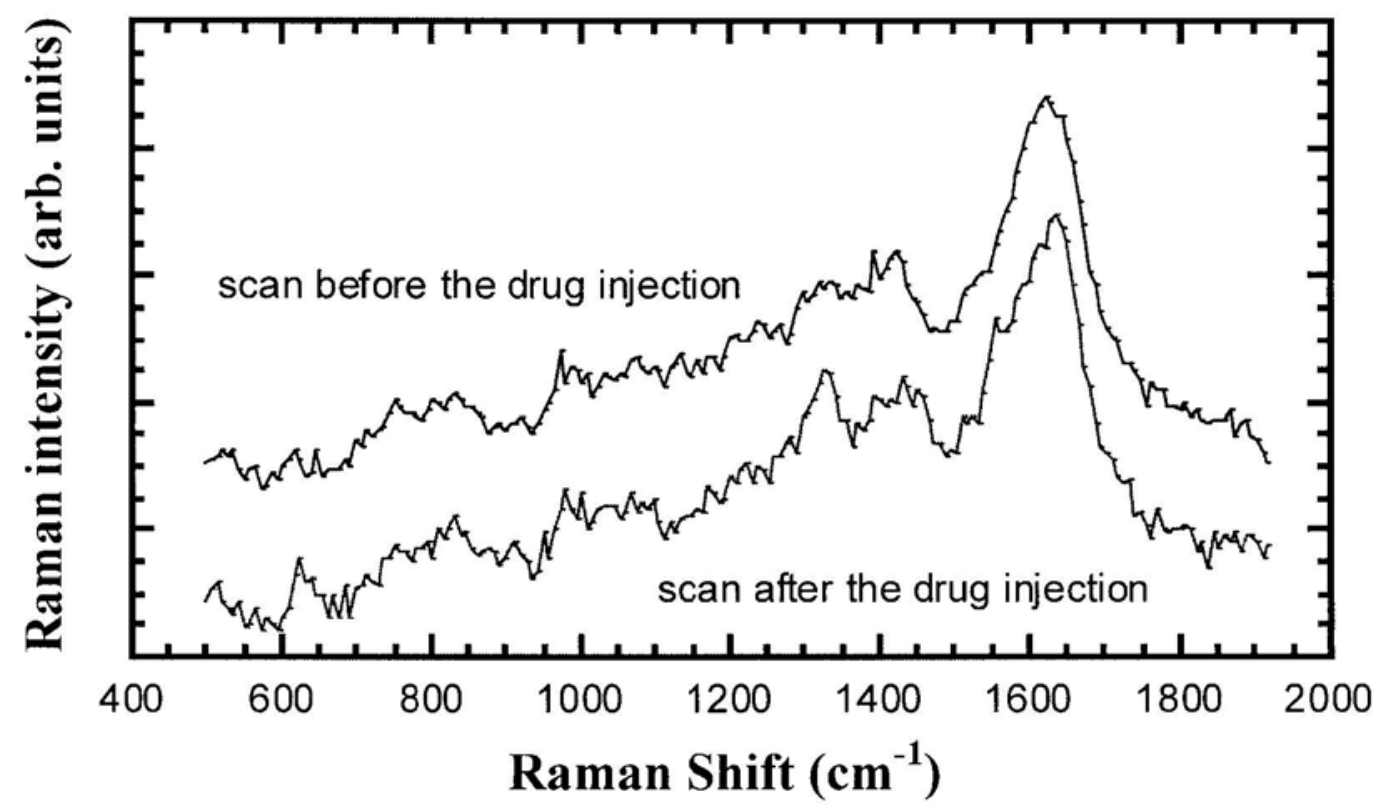

Figure 4a. Raman spectra of the anterior chamber before and after injection of 100 microgram Ganciclovir

The onset, duration, and intensity of a drug's actions are in turn modified by these interactions with physiological and biochemical functions. Raman spectroscopy may offer an effective tool to noninvasively assess the concentration of ganciclovir in the aqueous humor. Bauer et al. have already shown the feasibility of Raman spectroscopy with the drug Trusopt in a pharmacokinetic study ${ }^{12}$. We suggest that Raman spectroscopy could potentially be used to determine the amount and the rate of drug release either from implants or from direct intravitreal injection locally within the eye. The availability of such data could enable the ophthalmologist to improve treatment efficacy by avoiding premature or late surgical replacement of the implants and intravitreal injections. The origin of the Raman peaks of ganciclovir are related to the intra-atomic bonds and the shape of the whole molecule. For instance, the strong Raman peak in the region close to $1586 \mathrm{~cm}^{-1}$ could be due to $\mathrm{C}=\mathrm{O}$ stretching mode. The 
$\mathrm{C}=\mathrm{O}$ stretching vibration usually appears as a prominent band close to $1600 \mathrm{~cm}^{-1}$ and also in higher regions. Other peaks obtained in the Raman spectrum of ganciclovir are most likely due to various $\mathrm{C}-\mathrm{H}$ intra-atomic bonds.

As we have shown, this technique has a high specificity for ganciclovir molecules. The presence of the drug among all other solvents in the anterior chamber can be detected and the concentration of the drug can be measured based on the intensity of its Raman peak. Furthermore, we observed unique spectral features of ganciclovir in the vitreous chamber of rabbit eye in vivo. Adjusting and adapting the optics in the apparatus could enable us to optimally focus the laser into the vitreous chamber where the drug is initially released to interact with retinal and other ocular cells. This is more easily accomplished with a human eye than with the rabbit eyes that were included in our study. The rabbit eye, being myopic in nature, has a lens that is several times thicker than a human lens. This makes the optical pathway through the rabbit lens considerably longer than that of a human lens and therefore affects the inherently weak Raman signal.

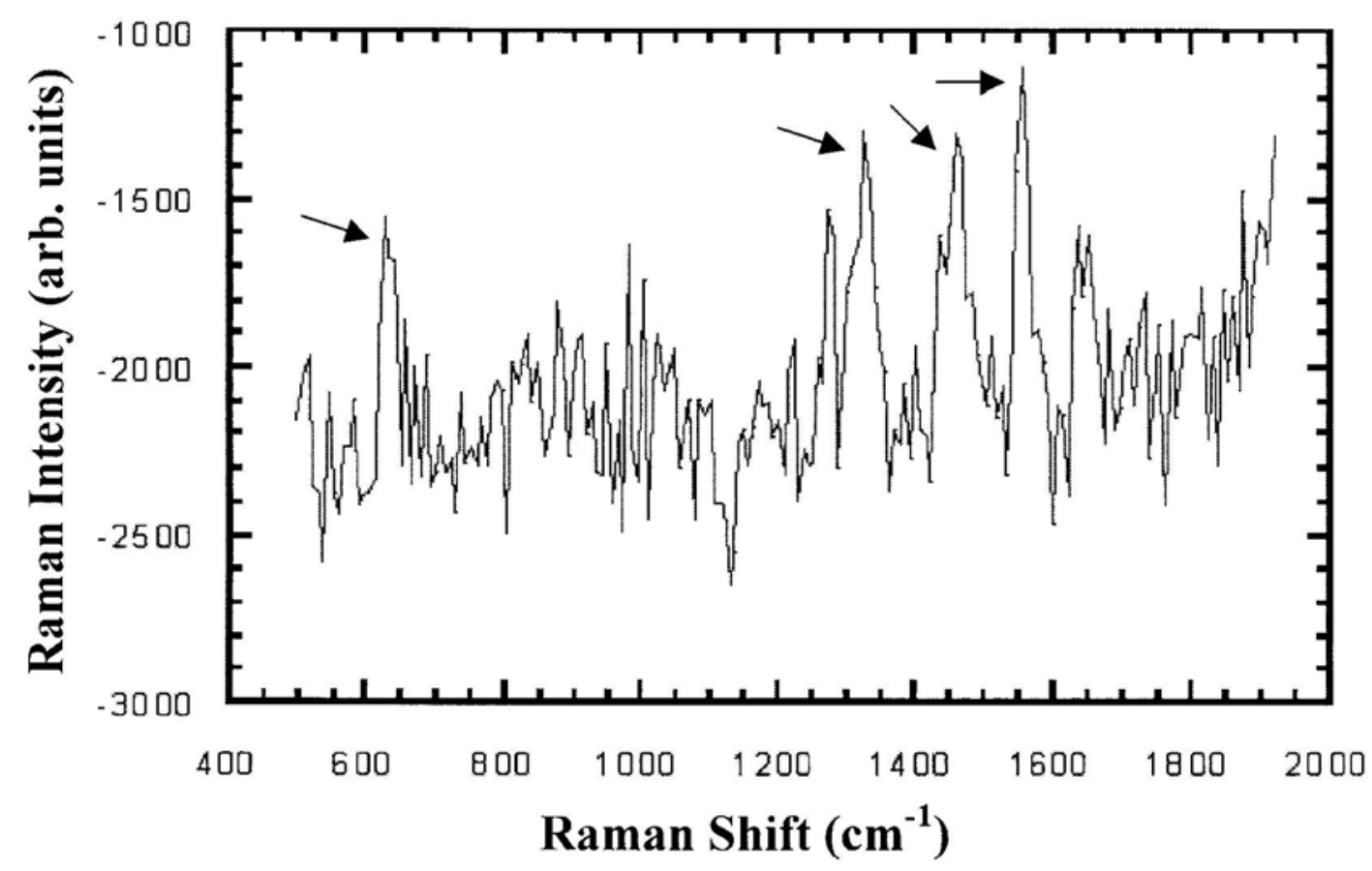

Figure 4b. Subtraction of the two spectra. Black arrows show the Ganciclovir peaks. 
Our study suggests that by increasing the sensitivity of Conforam, lower concentrations of ganciclovir that approximate therapeutic levels could easily be measured. The future development of more sensitive CCD chips and increasingly sophisticated detection software could result in the detection of even lower concentrations of the drug. Another way to enhance the detection power of the system is to employ a higher laser power and/or a longer exposure time.

A higher laser power would stimulate a stronger Raman signal emission.

Further improvements in the signal to noise ratio could be achieved by prolonging the exposure time of the ocular media to the laser beam. The addition of several seconds to the detection mode would greatly enhance the sensitivity of the system.

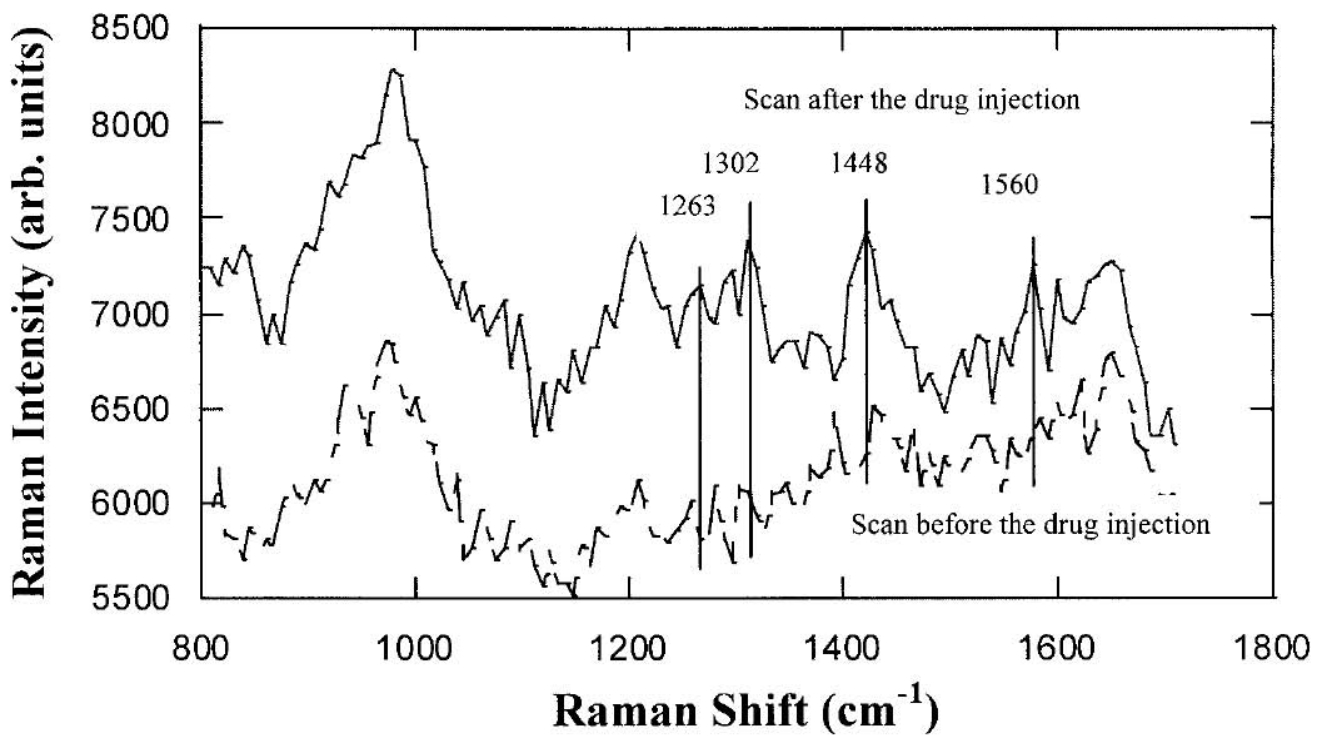

Figure 5. Raman spectra of the vitreous chamber before and after injection of 2000 microgram Ganciclovir. Black lines represent the Ganciclovir peaks

At this point we deliberately chose not to apply these measures simply for issues of safety stemming from the use of a laser beam of $514.5 \mathrm{~nm}$ wavelength in the ocular media and its potential hazards to the retinal layer of the eye. Once a safer laser source such as NIR laser beam, is diverted so that a minimum of direct laser light is expected to irradiate the retinal layer, more power and longer exposure time could potentially be applied in order to enhance the detection sensitivity of the system. The detection limit of Conforam could also be further improved by minimizing fluorescence that originates from ocular media that tends to diminish the signal-to-noise ratio. A "two wavelength" approach could enhance the Raman signal detection of lower concentrations of a drug, thereby reducing noise associated with tissue fluorescence. Borchert et. al. have successfully applied a two wavelength Raman spectroscopy technique in a glucose monitoring study in rabbit aqueous humor medium. They greatly eliminated broadband fluorescence by subtracting the 
scans acquired with two slightly different wavelengths from one another ${ }^{8}$. Another area to which this technique can potentially be applied is the study of biodegradable polymers, which are being employed to develop new forms of drug releasing implants. The noninvasive character of this technique allows the function of these implants to be monitored over time once they have been placed in an in vivo environment. Other techniques require direct invasive probing of the implant in order to acquire and analyze samples, thereby causing artifacts and disturbing the system. Our approach, however, leaves the system intact and allows the researcher to study the behavior, release, and even the biodegradable shell of a drug simultaneously.

\section{Acknowledgements}

The authors thank Dr. Thall for his contribution in this paper. Supported in part by Research to Prevent Blindness, Inc. 


\section{References}

1. Kishore, K., Conway, M.D. and Peyman, G.A. Intravitreal clindamycin and dexamethasone for toxoplasmic retinochoroiditis. Ophthalmic Surg. Lasers 32(3):183-192, 2001.

2. Yasukawa, T., Kimura, H., Kunou, N., Miyamoto, H., Honda, Y., Ogura, Y. and Ikada, Y. Biodegradable scleral implant for intravitreal controlled release of ganciclovir. Graefes Arch. Clin. Exp. Ophthalmol. 238(2):186-190, 2000.

3. Waga, J. Ganciclovir delivery through an intravitreal microdialysis probe in rabbit. Acta Ophthalmol. Scand. 78(3):369-371, 2000.

4. Martin, D.F., Ferris, F.L., Parker, D.J., Walton, R.C., Mellow, S.D., Gibbs, D., Walton, R.C., Mellow, S.D., Gibbs, D., Remaley, N.A., Ashton, P., Davis, M.D., Chan, C.C. and Nussenblatt, R.B. Ganciclovir implant exchange.

5. Timing, surgical procedure, and complications. Arch. Ophthalmol. 115(11):13891394, 1997.

6. Lim, J.I., Wolitz, R.A., Dowling, A.H., Bloom, H.R., Irvine, A.R. and Schwartz, D.M. Visual and anatomic outcomes associated with posterior segment complications after ganciclovir implant procedures in patients with AIDS and cytomegalovirus retinitis. Am. J. Ophthalmol. 127(3): 288-293, 1999.

7. Mietz, H., Aisenbrey, S., Ulrich BartzSchmidt, K., Bamborschke, S. and Kriegelstein, G.A. Ganciclovir for the treatment of anterior uveitis. Graefes Arch. Clin. Exp. Ophthalmol. 238(11): 905-909, 2000.

8. Luu, K.K., Scott, I.U., Chaudhry, N.A., Verm, A. and Davis, J.L. Intravitreal antiviral injections as adjunctive therapy in the management of immunocompetent patients with necrotizing herpetic retinopathy. Am. J. Ophthalmol. 129(6):811$813,2000$.

9. Borchert, M.S., StorrieLombardi, M.C. and Lambert, J.L. A noninvasive glucose monitor: preliminary results in rabbits. Diabetes Technol. Ther. 1(2):145-151, 1999.

10. Caspers, P.J., Lucassen, G.W., Carter, E.A., Bruining, H.A. and Puppels, G.J. In vivo confocal Raman microspectroscopy of the skin: noninvasive determination of molecular concentration profiles. J. Invest. Dermatol. 116(3):434-442, 2001.

11. Moseley, H., Foulds, W.S., Allan, D. and Kyle, P.M. Routes of clearance of radioactive water from the rabbit vitreous. Br. J. Ophthalmol. 68(3):145-151, 1984. 
12. WelgeLussen, L. and Schimmel, K.P. Examinations of aminoacids in aqueous humour after intravitreous injection of ascorbic acid. Klin. Monatsbl. Augenheilkd. 172(6):824-830, 1978.

13. Bauer, N.J., Motamedi, M., Wicksted, J.P., March, W.F., Webers, C.A. and Hendrikse, F. Noninvasive assessment of ocular pharmacokinetics using Confocal Raman Spectroscopy. J. Ocul. Pharmacol. Ther. 15(2):123-134, 1999. 


\section{Chapter 3}

\section{Non-Invasive Monitoring of Commonly Used Intraocular Drugs Against Endophthalmitis by Raman Spectroscopy}

Lasers in Surgery and Medicine 32:265-270 (2003)

K. Hosseini, $\mathrm{MD}^{1,2,3}$, F.H.M. Jongsma, $\mathrm{PhD}^{3}$, F. Hendrikse, $\mathrm{MD}, \mathrm{PhD}^{3}$ and $\mathrm{M}$. Motamedi, $\mathrm{PhD}^{1,2}$

\footnotetext{
The University of Texas Medical Branch, The Center for Biomedical Engineering, Galveston, Texas Department of Ophthalmology and Visual Sciences, Galveston, Texas

Department of Ophthalmology, AZM, Maastricht, The Netherlands
} 


\begin{abstract}
Purpose: To develop a non-contact and noninvasive method for quantification of the local concentration of certain antibiotic and antifungal drugs in the eye.

Study Design/Materials and

Methods: An integrated CCD-based Raman spectroscopic system designed specifically

for ophthalmic applications was used to noninvasively detect the presence of Ceftazidime and Amphotericin B in ocular media. Specific Raman signatures of the above named drugs were determined for various concentrations that were injected through a needle in the aqueous humor of rabbit eyes in vivo. Raman spectra were subsequently acquired by focusing an argon laser beam within the anterior chamber of the eye.

Results: Compared to ocular tissue, unique spectral features of Ceftazidime appeared near $1028,1506,1586$, and $1641 \mathrm{~cm}^{-1}$. Amphotericin B exhibited its characteristic peaks at 1156.5 and $1556 \mathrm{~cm}^{-1}$. The amplitudes of the spectral peak corresponding to these drugs (acquired by 1 second exposure time and $25 \mathrm{~mW}$ of laser power) were determined to be linearly dependent on their local concentration in the anterior chamber of the eye.

Conclusions: Raman spectroscopy may offer an effective tool to noninvasively assess the local concentration of the delivered drugs within the ocular media. This technique potentially could be used to investigate the pharmacokinetics of intraocular drugs in vivo either from a releasing implant or a direct injection.
\end{abstract}




\section{Introduction}

Endophthalmitis is one of the serious inflammatory ocular diseases, which could easily lead to blindness if left untreated. It can be grossly divided into endogenous and exogenous groups with a further subdivision into bacterial and fungal groups based on the causative microorganisms. Endogenous endophthalmitis is not an uncommon disease and there has been an increase in its appearance in recent decades in the US and prospective studies of hospitalized patients with candidemia showed that a remarkable 9-37\% of patients developed candidal endophthalmitis ${ }^{1}$. A high number of microorganisms capable of causing it emphasize the importance and potential hazards of this disease. The occurrence of this disease after different surgical interventions has also been documented in recent years ${ }^{2-4}$.

Local drug delivery in the eye offers the potential to significantly enhance the efficacy of treatment for endophthalmitis and other ocular pathological conditions, while reducing the side effects and toxicity associated with systemic applications of these drugs at concentrations that will be needed to effectively treat this and other ocular diseases. Direct injection of drugs into the eye is the primary approach for local drug delivery in the ocular medium. Other means such as surgical placement of drug releasing implants have also been utilized in recent years ${ }^{5,6}$.

In order for these approaches to be deemed safe and effective, accurate knowledge of actual drug dose and its rate distribution as well as elimination is critical. Ceftazidime, a third generation cephalosporine, has been advocated by many researchers and physicians in the recent years as a potentially safer substitute for Amikacin in treatment of bacterial endophthalmitis in order to minimize retinal toxicity. It is administered both as intravitreal and subconjuctival injections.

Management of various fungal infections such as fungal endophthalmitis is realized by careful dosing of Amphotericin B. This drug is a highly toxic yet widely used antifungal agent produced by Streptomyces nodosus. Due to the notorious toxicity of Amphotericin $\mathrm{B}$, many medical and scientific authorities urge that it be used under close supervision.

At present there is no means of evaluating the function of an implant or the concentration of the injected drug once the implant has been surgically placed in the eye other than invasively sampling aqueous or vitreous humor through needles. The lack of any real-time monitoring of drug concentration significantly limits the ability of the ophthalmologist to optimize treatment efficacy for each individual and to determine the optimum time for a subsequent injection or replacement of the implant. Thus, application of a non-invasive approach capable of quantifying local drug concentration within the eye is highly desirable because it can eliminate the risks involved with acquiring samples through needles while providing a mean for continuous monitoring of drug release.

In the biomedical field, the presence of molecules that exhibit unique Raman signatures can be detected using Raman spectroscopy ${ }^{7-11}$. Raman spectroscopy offers the potential to detect a specific molecule based upon the characterization of the vibrational frequencies of various molecules ${ }^{12}$. This technique has already been successfully utilized to monitor the presence of various concentrations of the antiviral drug Ganciclovir within 
the ocular media ${ }^{13}$. It has already been demonstrated that intravitreally administered substances penetrate into the anterior chamber of the eye ${ }^{14,15}$.

We have identified Raman signatures for the above named drugs and non-invasively detected and quantified the concentrations of these agents in the anterior chamber of white New Zealand rabbit eyes in order to explore the potential of Raman spectroscopy for real-time assessment of pharmacokinetics of Ceftazidime, Amphotericin B in vivo.

\section{Materials and methods}

Figure 1 shows a schematic drawing of an integrated CCD based scanning confocal Raman spectroscopy system with a long working distance for the noninvasive biochemical characterization of ocular tissue. The key components of the system are a single grating spectrometer with a focal length of $0.5 \mathrm{~m}$ and an aperture of $\mathrm{F}: 4$ (SPEX500M; spex industries, Edison, NJ) with a nitrogen-cooled CCD camera with high quantum efficiency for rapid signal detection, an argon-ion laser emitting linearly polarized beam at $514.5 \mathrm{~nm}$, a dielectric filter positioned at $45^{\circ}$ with respect to the laser beam served as a band filter for the incident polarized laser beam. A long working distance microscope objective lens (magnification $\mathrm{x} 25$; numerical aperture $=0.5$; focal length $=10 \mathrm{~mm}$; Jena, Karl Zeiss, Oberkochen, Germany) that acts as a focusing device for the incident light and the collecting lens for the Raman back-scattered light. This lens permits noninvasive probing of a considerable axial distance that reaches the vitreous chamber of the rabbit eye. An optical fiber coupled to the spectrometer collects the Raman back-scattered light and acts similar to a confocal pinhole.

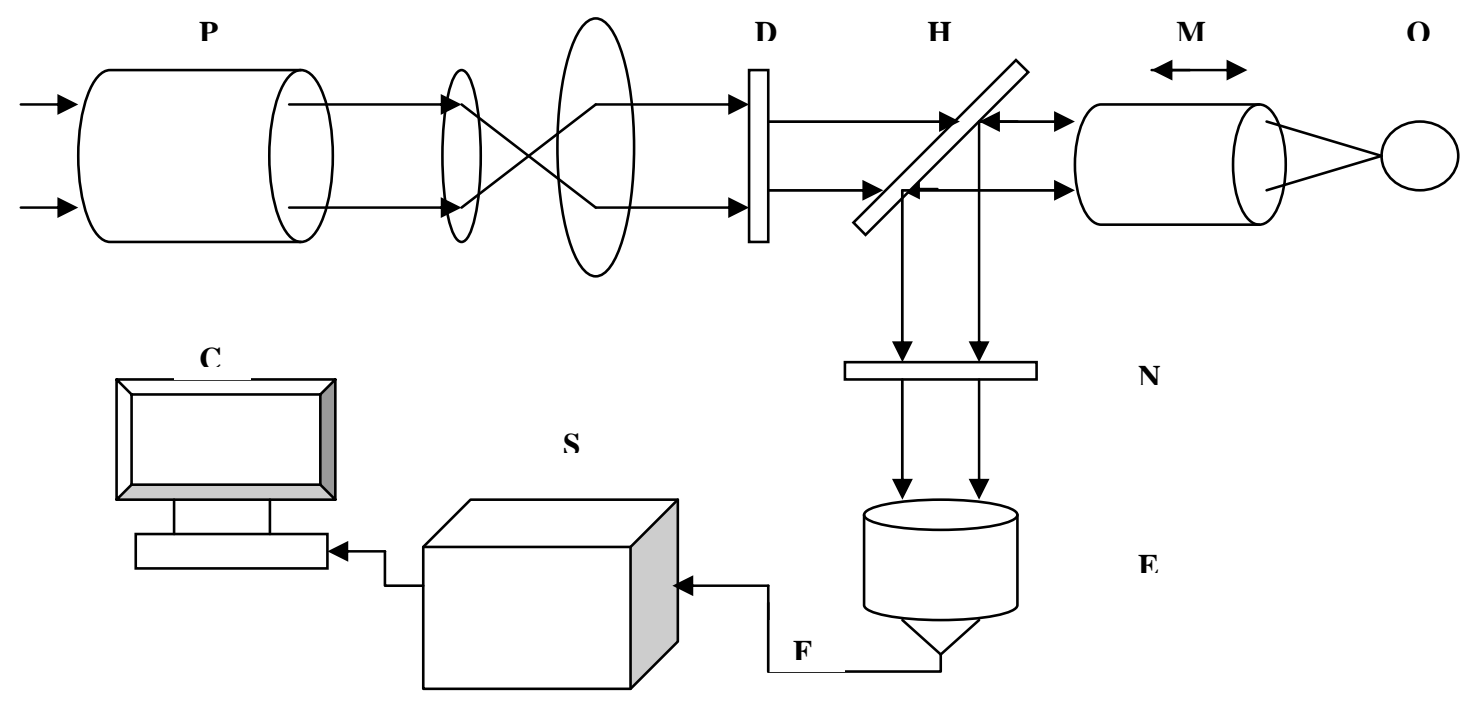

Figure 1. Confocal Raman scattering setup consisting of polarization rotator $(P)$, beam expander (B), diaphragm (D), band pass filter/Raman scattered light reflector $(H)$, microscope objective $(M)$, eye $(O)$, notch filter $(N)$, exit lens $(E)$, fiber probe $(F)$, spectrometer $(S)$, and computer $(C)$. 
The Raman signatures of Ceftazidime (Fortaz, Glaxo Wellcome) and Amphotericin B, as well as the magnitude of their spectral peaks were characterized by separately dissolving various concentrations (respectively $2.5-40 \mathrm{mg}, 0.375-6 \mathrm{mg}$ ) of these drugs in de-ionized water and placing each solution in a quartz cuvette that was probed with an argon laser beam $(25 \mathrm{~mW}, 1$ second).

Enhancement of the signal-to-noise ratio of the inherently weak Raman signal of this media was achieved by "binning', the CCD-camera. Binning (by the factor 5 on $\mathrm{X}$-axis of the chip for the experiments in the anterior chamber) improves the signal-tonoise ratio by combining the charge in adjacent pixels and increasing the intensity by an amount proportional to the number of pixels binned. The spectrometer's grating was set to acquire the Raman signals within the range of $600-1900 \mathrm{~cm}^{-1}$.

The rabbits were anesthetized using a mixture of Ketamine and Xylazine injected intramuscularly $(\mathrm{n}=10$, eight eyes per drug and four eyes per each drug concentration, eight eyes for anterior chamber and two eyes for vitreous chamber). We acquired Raman spectra of the aqueous humor of white New Zealand rabbits in vivo while keeping the grating range the same as the in vitro studies. All animal care and treatment followed humane laboratory methods, as described in the Guide of Care and Use of Laboratory Animals (NIH Publication \#86-23) and approved by the Institutional Animal Care and Use Committee of the University of Texas Medical Branch at Galveston.

We have utilized a mobile table (in two axes), which enables us to position the rabbits accurately so that the probing of the anterior chamber by the laser beam could be achieved. Using the specific Raman signatures of the cornea and lens in the region of $2840-3000 \mathrm{~cm}^{-1}$ ensures the right positioning of the probing beam in between these two ocular media, which is filled with the aqueous humor. We scanned and mapped the native Raman peaks of the anterior chamber prior to the injections of the intraocular drugs in this media. Then we tapped a pre-determined volume $(0.02-0.10 \mathrm{ml})$ of aqueous humor via paracentesis and replaced it with an identical volume containing two different concentrations of each drug in a separate experiment ensuring their presence in the anterior chamber with a known drug level. Subsequently, a series of specific Raman signatures were acquired for the differing amounts (0.4-2 mg of Ceftazidime and 30-90 mg of Amphotericin B) that had been injected in the aqueous humor of rabbit eyes in vivo. We also injected a higher dose of each drug through the pars plana so that the detection ability of our setup in vitreous chamber could be assessed. For each drug, one eye was tested in a subject that was not previously enrolled in the experiments designed to assess drug concentration within the anterior chamber. 


\section{Results}

The Raman spectra of Ceftazidime and Amphotericin B in the range of 600-1800 $\mathrm{cm}^{-1}$ are shown in figure 2. For visualization purposes the spectra were displaced on $\mathrm{Y}$ axis. The correlations between the intensity of the Raman signal for Ceftazidime in the range of 1451-1702 cm-1 and Amphotericin B in 1557-1579 cm-1 are determined by the area under the curve in these regions versus various drug concentrations as shown in figure 3 .

In the in vivo studies, Raman spectra of the aqueous humor of white New Zealand rabbits were acquired using the same grating and laser power setup as in the in vitro studies. Figure 4a,b display the spectra of the anterior chamber before and after the injection of each drug in this ocular space. The occurrence of small spectral features in the anterior chamber of the eye which are native to this medium were to be expected since different biological molecules with various Raman activity already exist in this medium $^{11,13}$. In order to be able to distinguish the specific peaks of the antibiotic Ceftazidime and antifungal Amphotericin B, we had to search for those peaks specific to these drugs that would not coincide with the inherent peaks of the aqueous humor. Figure 2 exhibits a few spectral peaks in the range of $600-1900 \mathrm{~cm}^{-1}$ for Ceftazidime, while Amphotericin B has only two very distinct peaks in this region.

After scanning the anterior chamber of the eye containing a certain concentration of Ceftazidime, the unique spectral features of this antibiotic appeared near 1028, 1506, 1586 (the strongest peak), and $1641 \mathrm{~cm}^{-1}$. For Amphotericin B we noted 1156.5 and 1556 $\mathrm{cm}^{-1}$ as its characteristic peaks. Again, in the in vivo studies, the magnitude of the spectral peaks corresponding to these drugs was determined to be linearly dependent on the local concentration of these agents in the anterior chamber of the eye. In order to understand the assessment power of Raman spectroscopy to monitor the concentration of these drugs non-invasively in vivo one should consider the results of the measurements performed based on estimating the same area under the curve as for data reported in figure 3. 


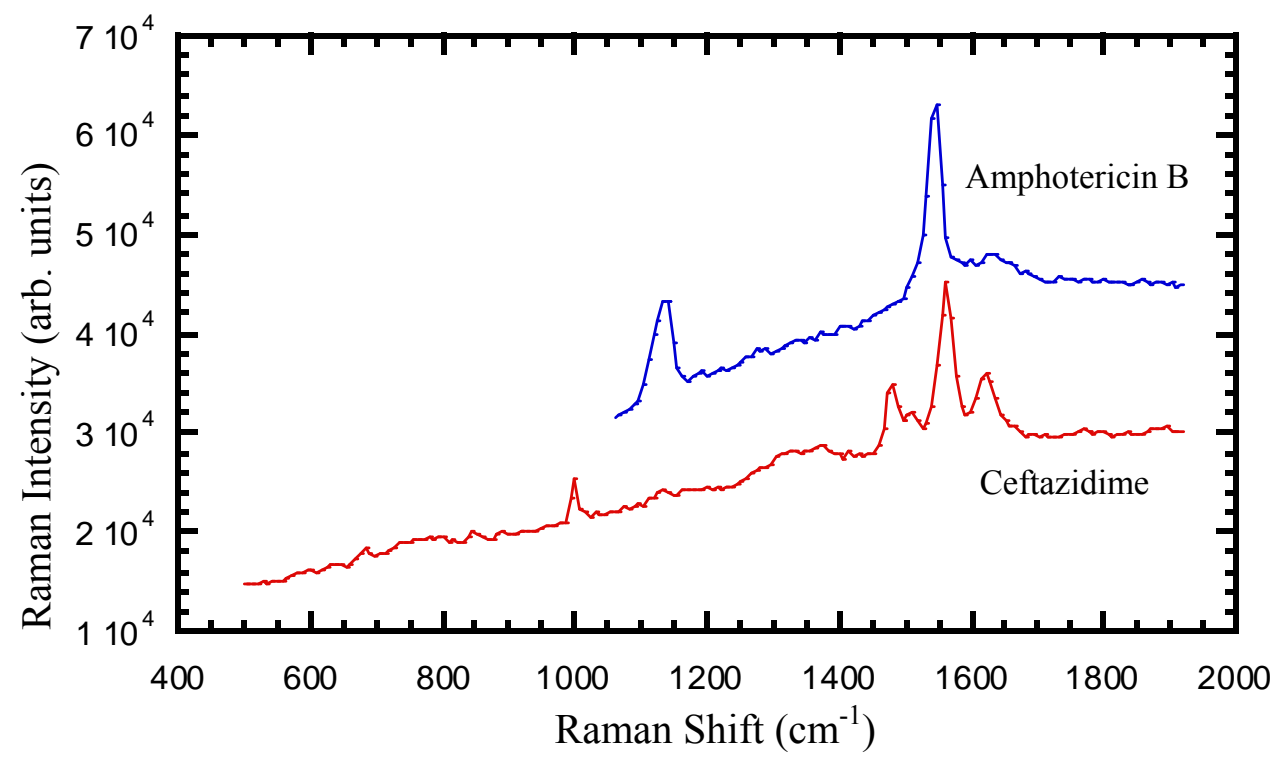

Figure 2. Raman spectra of Ceftazidime and Amphotericin B.

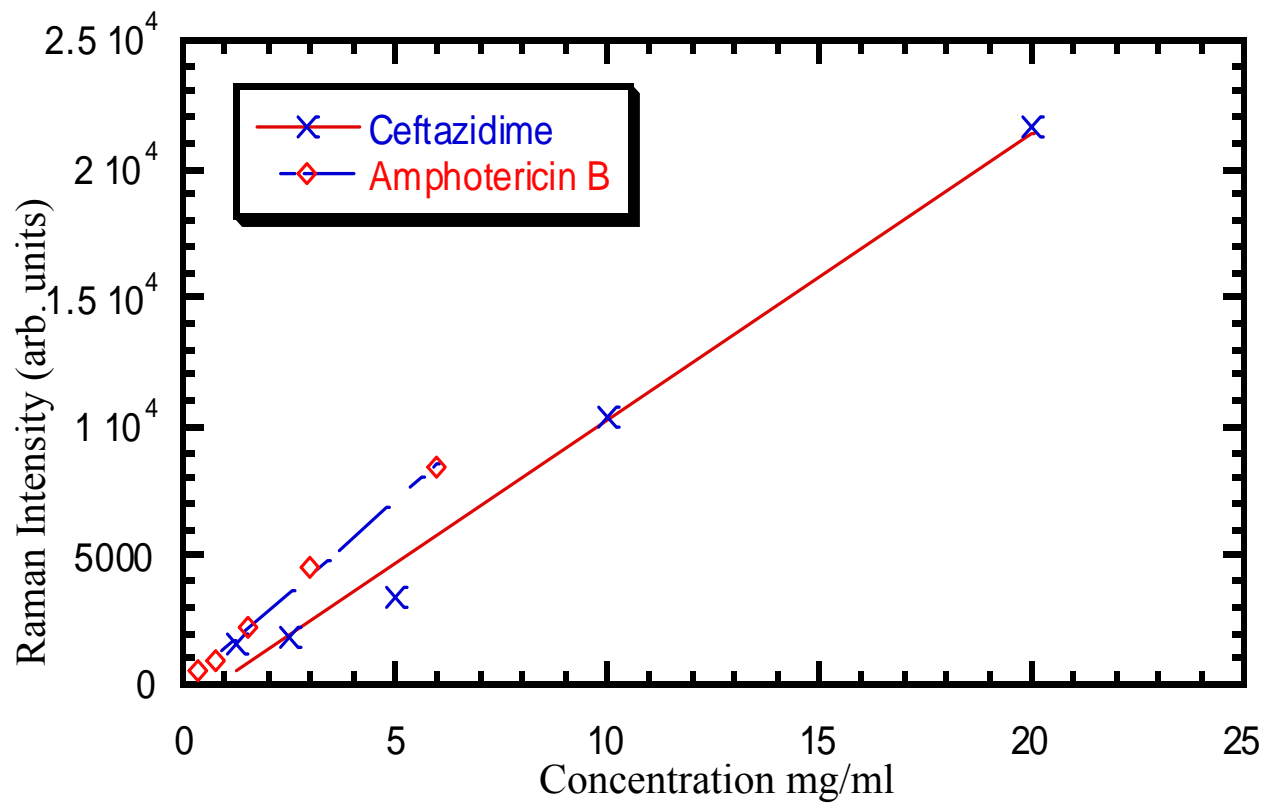

Figure 3. Raman intensity versus different concentrations of Ceftazidime and Amphotericin B in quartz cuvettes assessed in an experimental setup. 

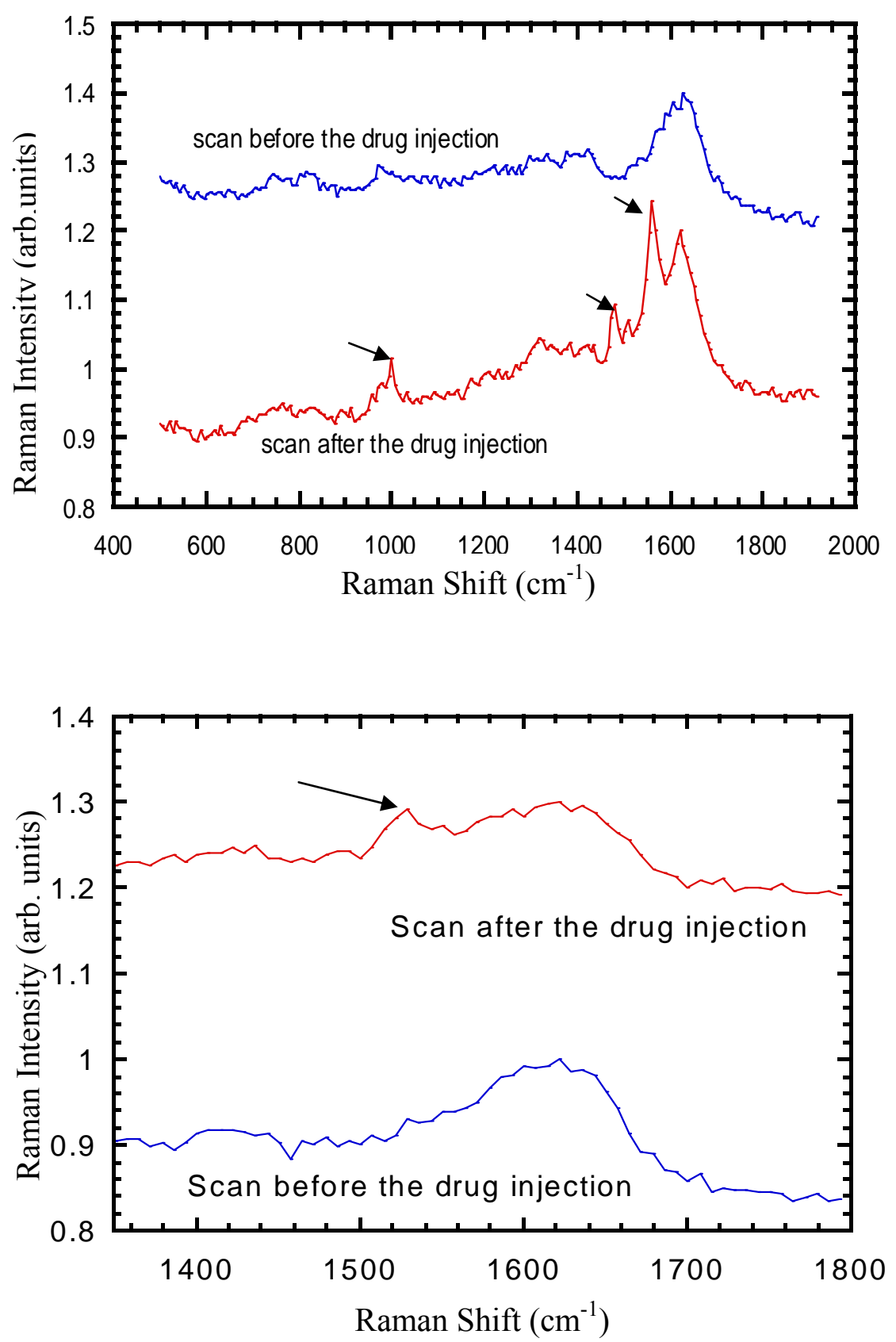

Figure 4.a: Raman spectra of the anterior chamber before and after injection of $2.5 \mathrm{mg}$ Ceftazidime. Black arrows indicate the Raman peaks. $b$ : Raman spectra of the anterior chamber before and after injection of $30 \mathrm{mg}$ Amphotericin B. Black arrow indicates the Raman peak. 
The statistical analysis and calculations for Amphotericin B (30 mg group's average is $2,821 \pm 941$ and the $90 \mathrm{mg}$ group's average is 11,321 $\pm 1,261)$ and Ceftazidime $(0.4 \mathrm{mg}$ group's average is $1.13 \mathrm{e}+05 \pm 32,872$ and the $2 \mathrm{mg}$ group's average is $2.46 \mathrm{e}+05 \pm 38,235$ ) yielded results which show the disparity between the numbers of different concentrations for each drug and supports the ability of Raman spectroscopy to non-invasively detect these agents as well. Figure 5a shows a Raman spectrum obtained following intravitreal injection of $5 \mathrm{mg}$ Ceftazidime through pars plana in the vitreous chamber of the eye. The strongest peak of Ceftazidime is still clearly visible in the scan, showing the possibility of detecting this drug within the vitreous chamber of the eye while the laser beam passes through the cornea, anterior chamber, and lens. The acquisition of this scan was realized without manually binning the chip to a higher number (bin $X=1$ ). This results in a better resolution of the detected peak, while making the background noisier than other spectra with a higher binning number, shown in this paper. Similar results were obtained for Amphotericin B (Fig. 5b, $1 \mathrm{mg}$, bin $X=2$ ), thus supporting the feasibility of this technique in detecting these agents in the vitreous chamber. 

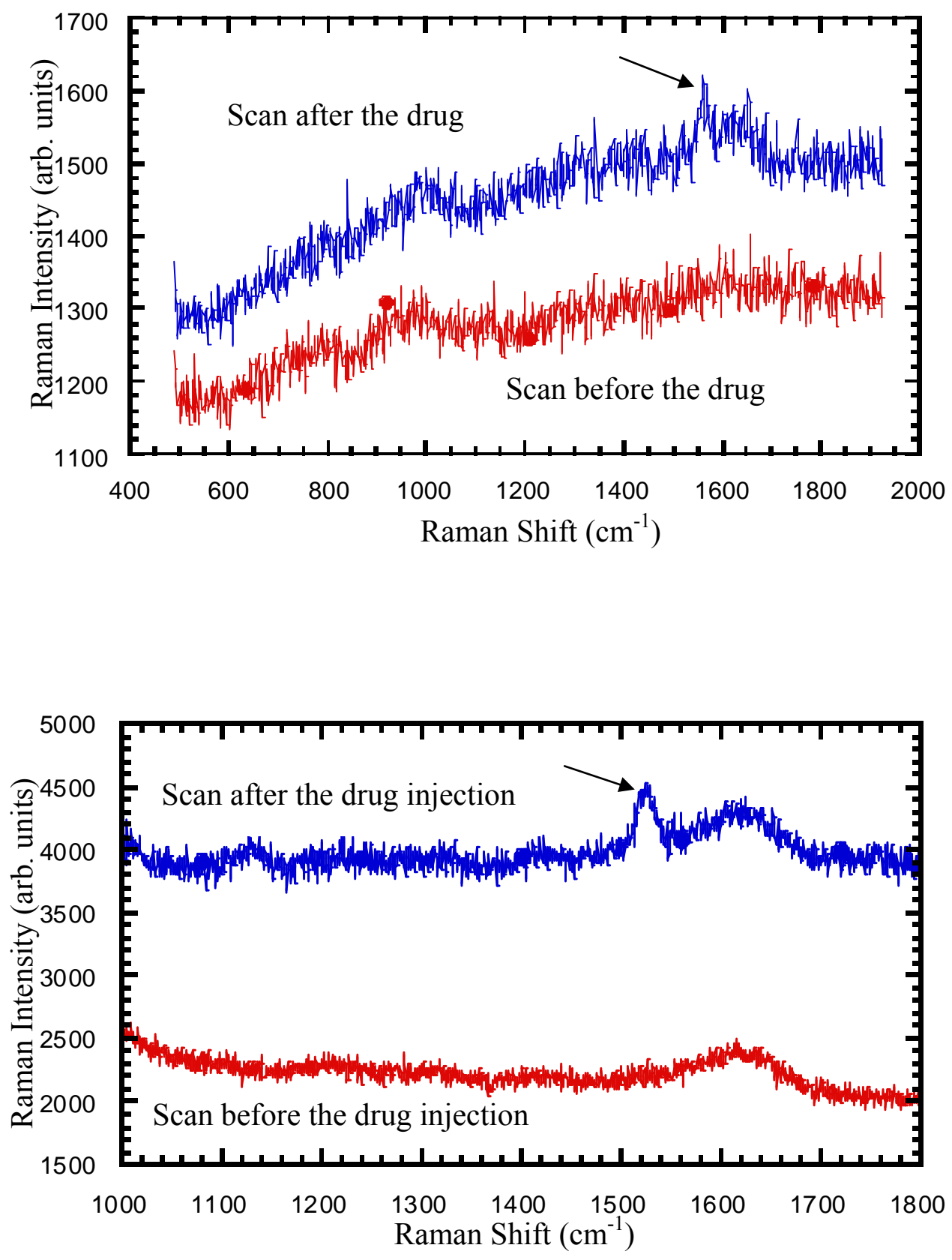

Figure 5. a: Raman spectra of the vitreous chamber before and after injection of $5 \mathrm{mg}$ Ceftazidime. Black arrow represents the Ceftazidime peak. $b$ : Raman spectra of the vitreous chamber before and after injection of $1 \mathrm{mg}$ Amphotericin B. Black arrow represents the Amphotericin B peak. 


\section{Discussion}

Management of endophthalmitis is a challenging task, with variable prognosis depending on the causative microorganism. While systemic administration of drugs has a relative higher threshold of reaching the toxicity levels, the intravitreal administration of drugs is even limited to a much narrower index. Ceftazidime is one of a few antibiotics used in bacterial endophthalmitis, while Amphotericin B forms still the mainstay drug in the management of fungal endophthalmitis. The goal is to intervene and administer the drug locally as fast as possible, make sure that the drug concentration level stays above the required therapeutic dose yet not exceed the vicious toxicity, which is fairly destructive to the sensitive retinal cells. The use of Amphotericin B against fungal endophthalmitis may have even a bigger dilemma than other intravitreal drugs due to its inherent strong toxicity. Although the reports in using liposome-intercalated Amphotericin B instead of the its regular form has proven to be less toxic and thus safer to re-intervene ${ }^{16}$, yet its extreme toxicity properties to the retinal cells of the eye makes the evaluation of a second or third injection into the eye in persistent cases a rather hard decision to make. Once again, a non-invasive method acquiring real-time data regarding the actual concentration of the drug within the ocular media would help the clinician to make more accurate therapeutic decisions.

Non-invasive and non-contact monitoring of a drug's presence or clearance rate would also be highly desirable because its concentration in the ocular media is subjected to unpredictable changes for every individual arising from different physiological and pathological conditions. Walstad et al. found that the mean penetration of Ceftazidime into the aqueous humor of the eye with and without endophthalmitis was significantly

different ${ }^{17}$. From that study, one can fairly assume that the severity of endophthalmitis could also affect its pharmacodynamics within the ocular media. Shaarawy et al. concluded that procedures such as vitrectomy and lensectomy could significantly shorten the half-life of Ceftazidime after intravitreal injection and that inflammation increases clearance to a small degree in phakic and aphakic eyes ${ }^{18}$. Earlier Mochizuki et al. also found different rates of half-life for Ceftazidime by different conditions in an animal study $^{19}$. These findings support the necessity of a real time monitoring mean regarding the drug concentration in endophthalmitis cases. Raman spectroscopy has been successfully utilized in rabbit eyes for different types of investigations ${ }^{11,20}$ and it may offer an effective tool to non-invasively assess the concentration of various intravitreal drugs as tested in both the aqueous humor and vitreous humor. Bauer et al. have already shown the feasibility of Raman spectroscopy in detecting the drug Trusopt in the tear film of the eye in a pharmacokinetic study ${ }^{21}$ and we have demonstrated its potential in monitoring the antiviral drug Ganciclovir in a previous study ${ }^{13}$.

In this study, we were able to detect $30 \mathrm{mg}$ Amphotericin B within the anterior chamber of the eye, which is only six times the usual dose used in the clinic. For Ceftazidime, we detected easily $0.4 \mathrm{mg}$ concentration of this drug. This is lower than the $2.5 \mathrm{mg}$ clinical dose of this drug for intravitreal injection, while much higher 
concentrations (up to $100 \mathrm{mg}$ ) are being used by some clinics for subconjunctival injections of this antibiotic. The detectable concentrations in these experimental settings compared with the common clinical doses, further emphasizes the promising prospect of this technique. The origins of the Raman peaks of these pharmaceutical agents are related to the intra-atomic bonds and the three dimensional structure of the molecule. The $\mathrm{C}=\mathrm{O}$ stretching vibration usually appears as a prominent band close to $1600 \mathrm{~cm}^{-1}$ and also in higher regions. Therefore the high Raman peak close to $1586 \mathrm{~cm}^{-1}$ could be due to $\mathrm{C}=\mathrm{O}$ stretching mode. Other peaks obtained in the Raman spectrum of these drugs are most likely due to various $\mathrm{C}-\mathrm{H}$ intra-atomic bonds. We propose that Raman spectroscopy could potentially be utilized to quantify the amount and the rate of drug release either from implants or from intravitreal injections within the eye. The availability of such data could enable the ophthalmologist to address treatment efficacy by avoiding premature or late surgical replacement of the implants and intravitreal injections. However, one certainly needs to note that not every intravitreal drug possesses Raman signature. For example Vancomycin, which is also broadly used in the treatment of endophthalmitis and other diseases, did not show any significant Raman activity as observed in other experiments in our laboratory. Nevertheless, it may be possible to design drugs for the treatment of ocular diseases with distinct Raman signature that can be monitored noninvasively. Feasibility of this approach has already been demonstrated for other compounds $^{22,23}$.

Our results revealed that Raman spectroscopy has a high specificity for Ceftazidime and Amphotericin B molecules. Wicksted and Erckens have already shown that it is extremely difficult to investigate the presence of the normal biological solvents in the aqueous humor by this technique, partly because of their relative low concentrations $^{24,25}$. The drugs in this study were able to be distinguished from all other solvents present in the anterior chamber and their concentrations were determined based on the intensity of their Raman peaks. In addition, we observed spectral features unique to these agents in the vitreous chamber of the rabbit eye in vivo.

Adjusting the optics in our Raman system would permit optimal focusing of probing beam into the vitreous chamber where the drug is initially delivered to interact with retinal and other ocular cells. It could be done much easier with human eye, since human lens is much thinner than rabbit lens, making the optical pathway and conversions through this structure much shorter. By improving the sensitivity of Raman detection system, lower concentrations of the tested drugs that more closely approach therapeutic levels could easily be quantified. The fabrication of increasingly sensitive CCD chips and more advanced detection software would likely result in the accurate measurement of even lower chemical concentrations. Increasing scanning time and/or laser power can vastly increase the sensitivity of the system. Once safer laser sources such as NIR laser beams are incorporated into our system, more laser power and longer exposure time could potentially be applied in order to enhance the detection sensitivity of the system. Furthermore, the short exposure time (1 second) used in the current study is relatively brief for typical Raman measurements. In our proposed instrument, laser radiation (25 $\mathrm{mW}$ ) is first focused tightly into the anterior chamber of the eye. That means that the maximum power of $25 \mathrm{~mW}$ is available at the focal point of the Jena lens inside this 
medium. Once the focused laser beam travels beyond the anterior chamber, the light rays diverge rather rapidly and after passing through the lens they refract to an even larger angle. This defocusing of the light, results in the distribution of the laser energy over a much larger volume, which would bring the total energy delivered to the retina closer to the acceptable safe levels.

The study of biodegradable polymers is another arena in which this technique could potentially be applied. Biodegradable polymers are being investigated and utilized as a releasing shell for intraocular implants such as the Ganciclovir implant for AIDS patients and fluocinolone acetonide in the treatment of diabetic macular edema (DME) and posterior uveitis. The non-invasive and non-contact properties of Raman spectroscopy provide the means to monitor the performance of these implants over an extended period of time once they have been placed in an in vivo environment.

Calibrating of this setup for actual measurements in real patients can be achieved by different methods. Since the anterior and vitreous chamber of the eye are filled almost entirely by water ${ }^{26}$, a fast and equal distribution of an injected drug into these ocular media could be expected. Therefore, local probing of the drug concentration should be accurate. In clinical practice, many of the pathological cases of endophthalmitis and other ocular infections do occur unilaterally. In these cases, the scan from the healthy eye of the patient can provide the data for zero concentration (base) of the drug X, which is the subject of the measurement, while a scan acquired on the day of the intravitreal injection of the drug $\mathrm{X}$ with the known concentration could provide the scan for maximum concentration of the drug X. Another potential approach is to acquire a scan from the eye, which is about to receive an injection before the actual administration of the drug. A second scan should be acquired on the same day after the injection of the known concentration of the intravitreal drug. These two scans represent the zero level and the maximum level of the injected drug into the eye. Any subsequent scan acquired in days after the injection of the first dose, will show changes in the Raman intensity, which could provide a mean to calculate the remaining concentration within the ocular medium.

In conclusion, the current study demonstrates the feasibility of Raman spectroscopy for monitoring drug concentration in the ocular media; in particular, the potential application of this technique to detect and monitor the concentration of commonly used antibiotic and antifungal drugs in the eye non-invasively. However, further work is needed in order to improve the accuracy and safety of the proposed technique.

\section{Acknowledgements}

The authors thank Drs. March, Wicksted and Erckens for their helpful contributions. 


\section{References}

1. Brooks RG. Prospective study of Candida endophthalmitis in hospitalized patients with candidemia. Arch Intern Med 1989;149(10):2226-2228.

2. Eifrig CW, Flynn HW, Jr., Scott IU, Newton J. Acute-onset postoperative endophthalmitis: Review of incidence and visual outcomes (1995-2001). Ophthalmic Surg Lasers 2002; 33(5):373-378.

3. Frisch L, Dick HB. Incidence of endophthalmitis in ophthalmic surgery. Dev Ophthalmol 2002;33:191-198.

4. Ernest J, Rejmont L, Nemec P. Epidemic endophthalmitis after cataract surgery. Eur J Ophthalmol 2002;12(3):225- 227.

5. Kishore K, Conway MD, Peyman GA. Intravitreal clindamycin and dexamethasone for toxoplasmic retinochoroiditis. Ophthalmic Surg Lasers 2001;32(3):183-192.

6. Yasukawa T, Kimura H, Kunou N, Miyamoto H, Honda Y, Ogura Y, Ikada Y. Biodegradable scleral implant for intravitreal controlled release of ganciclovir. Graefes Arch Clin Exp Ophthalmol 2000;238(2):186-190.

7. Borchert MS, Storrie_Lombardi MC, Lambert JL. A noninvasive glucose monitor: Preliminary results in rabbits. Diabetes Technol Ther 1999;1(2):145151.

8. Caspers PJ, Lucassen GW, Carter EA, Bruining HA, Puppels GJ. In vivo confocal Raman microspectroscopy of the skin: Noninvasive determination of molecular concentration profiles. J Invest Dermatol 2001;116(3):434-442.

9. Bauer NJ, Wicksted JP, Jongsma FH, March WF, Hendrikse F, Motamedi M. Noninvasive assessment of the hydration gradient across the cornea using confocal Raman spectroscopy. Invest Ophthalmol Vis Sci 1998;39(5):831-835.

10. Koo TW, Berger AJ, Itzkan I, Horowitz G, Feld MS. Reagent-less blood analysis by near-infrared Raman spectroscopy. Diabetes Technol Ther 1999;1(2):153-157.

11. Erckens RJ, Jongsma FHM, Wicksted JP, Hendrikse F, March WF, Motamedi M. Raman Spectroscopy in Ophthalmology: From experimental tool to application in vivo. Lasers Med Sci 2001(Review);16(4):236-252.

12. Katz A, Kruger EF, Minko G, Liu CH, Rosen RB, Alfano RR. Detection of glutamate in the eye by Raman spectroscopy. SPIE Proceedings, Vol. 4098.nbm.

13. Hosseini K, March W, Jongsma FH, Hendrikse F, Motamedi M. Noninvasive detection of ganciclovir in ocular tissue by Raman spectroscopy: Implication for monitoring of drug release. J Ocul Pharmacol Ther 2002;18(3):277-285. 
14. Moseley H, Foulds WS, Allan D, Kyle PM. Routes of clearance of radioactive water from the rabbit vitreous. Br J Ophthalmol 1984;68(3):145-151.

15. Welge-Lussen L, Schimmel KP. Klin Monatsbl Augenheilkd 1978;172(6):824830 .

16. Barza M, Baum J, Tremblay C, Szoka F, D’Amico DJ. Ocular toxicity of intravitreally injected liposomal amphotericin B in rhesus monkeys. Am J Ophthalmol 1985;100(2): 259-263.

17. Walstad RA, Blika S, Thurmann-Nielsen E, Halvorsen TB. The penetration of ceftazidime into the inflamed rabbit eye. Scand J Infect Dis 1987;19(1):131-135.

18. Shaarawy A, Meredith TA, Kincaid M, Dick J, Aguilar E, Ritchie DJ, Reichley RM. Intraocular injection of ceftazidime. Effects of inflammation and surgery. Retina 1995;15(5):433-438.

19. Mochizuki K, Yamashita Y, Torisaki M, Komatsu M, Tanahashi T, Kawasaki K. Intraocular kinetics of ceftazidime (Modacin). Ophthalmic Res 1992;24(3):150_ 154.

20. Mizuno A, Tsuji M, Fuji K, Kawauchki K, Ozaki Y. Near-infrared Fourier transform Raman spectroscopic study of cornea and sclera. Jpn J Ophthalmol 1994;38(1):44-48.

21. Bauer NJ, Motamedi M, Wicksted JP, March WF, Webers CA, Hendrikse F. Noninvasive assessment of ocular pharmacokinetics using confocal Raman spectroscopy. J Ocul Pharmacol Ther 1999;15(2):123-134.

22. Sijtsema N, Duindam J, Puppels G, Otto C, Greve J. Imaging with extrinsic Raman labels. Applied Spectroscopy 1996;50: 545-551.

23. Carey P. Resonance Raman labels and Raman labels. J Raman Spectroscopy 1998;29:861-868.

24. Wicksted JP, Erckens RJ, Motamedi M, March WF. Raman spectroscopy studies of metabolic concentrations in aqueous humor specimens. Appl Spectrosc 1995;49:987.

25. Erckens RJ, Motamedi M, March WF, Wicksted JP. Raman spectroscopy for noninvasive characterization of ocular tissue: Potential for detection of biological molecules. J Raman Spectrosc 1997;28:293.

26. Prince JH. The rabbit in eye research. Thomas C Publisher; 1964. 


\section{Chapter 4}

\section{RAMAN SPECTROSCOPY: NONINVASIVE DETERMINATION OF SILICONE OIL IN THE EYE: Potential Applications for Intraocular Determination of Biomaterials}

RETINA: Volume 22(6) December 2002 pp 796-799

ERCKENS, ROEL J. MD, PhD*; HOSSEINI, KAMRAN MD†; MARCH, WAYNE F. MD†; JONGSMA, FRANCISCUS H.M. PhD*; WICKSTED, JAMES P. PhD ; Li, HELEN K. MD†; HENDRIKSE, FRED MD, PhD* 


\begin{abstract}
Purpose: To show in a case study that Raman spectroscopic analysis for noninvasive determination of intraocular molecular structures of tissue or artificial materials is feasible.

Material and methods: Raman spectroscopic recordings were obtained using a confocal detection probe with an adjustable integration depth to resolve a small droplet $(0.75 \mathrm{~mm})$ of silicone oil noninvasively in an animal model (rabbit eye) at different power settings. After these measurements, the power was reduced to $95 \mu \mathrm{W}$ for a safe measurement in the human eye.

Results: Raman spectral features indicative of silicone oil were detected at $2906 \mathrm{~cm}^{-1}$ and $2965 \mathrm{~cm}^{-1}$. In the rabbit model, silicone oil could be distinguished from cornea or lens tissue due to the typical Raman spectrum. Even with a safe $95 \mu \mathrm{W}$ power setting, silicone oil could be resolved in the anterior chamber of a patient's eye with a centrally opaque cornea.

Conclusion: Due to the strong intensity and specificity of the Raman signal of silicone oil, even small amounts, for instance, those present in the anterior chamber of the eye, can be detected within a time frame of seconds using a safe laser power setting. With these results, an initial step is made in exploring the use and eventually the clinical application of the confocal Raman spectroscopic technique to obtain in vivo information on biological or artificial molecules in the living eye.
\end{abstract}




\section{Introduction}

Intraocular biomaterials are extensively used $^{1}$, and well-known examples include the artificial intraocular lenses (IOL) and the vitreous substitutes like silicone oil. Silicone oil is used to tamponade the retinal tissue ${ }^{2}$. In past years, an increase in the use of silicone oil by the vitreoretinal surgeon while performing a pars plana vitrectomy (PPV) has been noticed. However, after the PPV, postoperative complications related to the use of the silicone oil may develop. A complete filling of the anterior chamber with the silicone oil is frequently difficult to determine at slit-lamp examination, particularly if the cornea is opaque, thus preventing observation of convection movements of particles in the anterior chamber or other visual signs indicative of the presence of silicone oil. The aim of this study was to investigate the use of Raman spectroscopy ${ }^{3}$, a noninvasive and therefore potentially safe technique to obtain information on the silicone oil in the anterior chamber of the eye. The Raman signal is formed by shifts of wavelengths of the incident light, which are related to the frequencies of the modes of vibrations of the lightscattering molecules. A confocal Raman excitation and collection probe for in vivo use has been developed ${ }^{4}$ and applied ${ }^{5,6}$ by our group.

\section{Materials and methods}

The Raman experimental setup with the confocal probe ${ }^{4}$ is shown in figure 1 . The main features of the probe are a non-contact measurement at a working distance of 12 $\mathrm{mm}$ by a long working distance microscope objective (LDMO) with a high lightgathering power and the ability to control the integration depth. This was achieved by a confocally placed collection fiber acting as the pinhole in the setup. In this case a fiber with a diameter of $400 \mu \mathrm{m}$ was applied, yielding an integration depth of approximately 1 $\mathrm{mm}$. A holographic bandpass filter allows the excitation light to pass, but reflects the Raman scattered light toward the fiber. An argon laser emitting at $514.5 \mathrm{~nm}$ was used to excite the Raman spectra. 


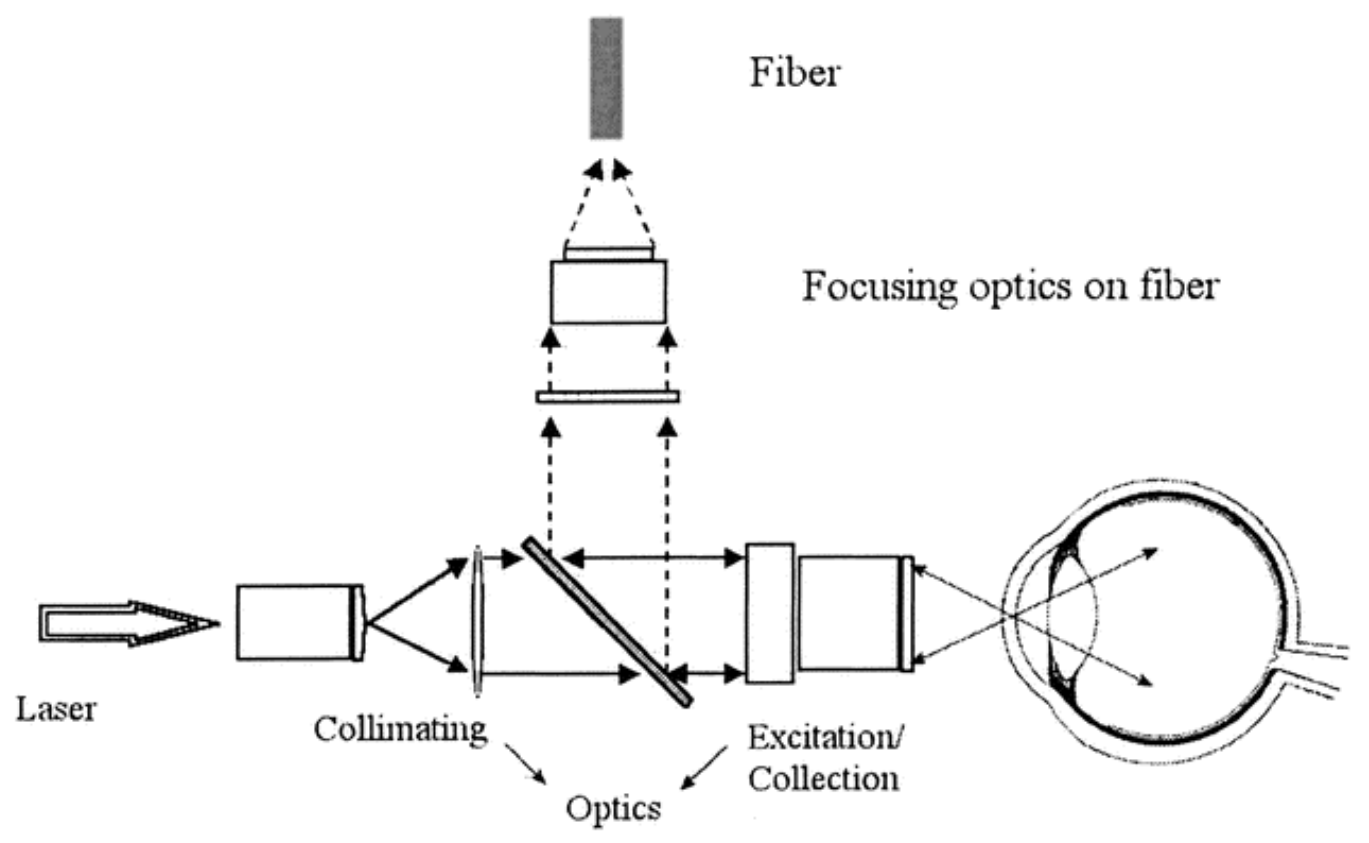

Figure 1. Raman experimental setup for in vivo measurements of silicone oil in the anterior chamber of the eye.

To determine the Raman signal of the silicone oil, a cuvette was filled with silicone oil with a viscosity of 5,000 centistokes (AdatoSil 5,000, Bausch \& Lomb Surgical, Heidelberg, Germany) and Raman spectra were recorded in 1 second using only $12 \mathrm{~mW}$. The feasibility and reliability of Raman spectroscopy in vivo determination of silicone oil was tested in an animal study. Two New Zealand white rabbits were anesthetized with ketamine and xylazine and treated according to the Association for Research and Vision and Ophthalmology (ARVO) resolution on the use of animals in vision research. The silicone oil was introduced in the anterior chamber by means of an injection at the limbus. In one eye of each animal, the amount of silicone oil injected was sufficient to obtain an almost complete filling of the anterior chamber. For these in vivo measurements, the laser power was reduced to $5 \mathrm{~mW}$ and 1 second integration time. For the experiment in the fellow eye, the laser power was further reduced to $0.5 \mathrm{~mW}$ and 1 second integration time. In this eye, a silicone oil bubble with a diameter of approximately $0.75 \mathrm{~mm}$ was injected at the limbus in the superior anterior chamber. The Raman spectrum was obtained by visual focusing on the cornea in front of the bubble. By moving the focusing beam through the different tissue layers, the cornea, silicone oil, and lens could be identified using their specific Raman spectra. In this eye, the pupil was dilated using a mydriatic agent (Mydfrin) to obtain the Raman spectrum of the lens. The animals were killed after the measurements with a lethal injection of intracardiac sodium pentobarbital once both eyes had been investigated. 


\section{Case report}

At the time, we had a 25-year-old male patient with silicone oil in the anterior chamber and a central opaque cornea. The patient had a traumatic event 7 months earlier owing to an exploding glass bottle. He received a PPV with silicone oil. The lens had to be removed during the PPV. He developed an early keratopathy, possibly due to corneal irritation from the silicone oil. The current vision in this eye was count fingers at 1 foot. The intraocular pressure was $11 \mathrm{mmHg}$. The study was performed under the approval of the institutional review board. The patient gave informed consent after the study protocol was explained. For proper positioning of the patient, a modified chin rest and forehead holder from a slit lamp were used and mounted on an optical bench, which allowed for height control, to support the head of the patient. Because the cornea of the patient was opaque in the center, the Raman spectrum was obtained on a fairly clear part of the inferior cornea. The laser power setting was $95 \mu \mathrm{W}$ with an integration time of 1 second. The American National Standard for the Safe Use of Lasers 7 gives maximum permissible exposure (MPE) to prevent laser light damage in tissues, including the retina. Using these safety standards, it is estimated that for the human, the MPE at a wavelength of $514.5 \mathrm{~nm}$ should be below $3 \mathrm{~mJ}$ if the illuminated retina area is approximately $2.7 \mathrm{~cm} 2$ with an exposure time equal to or longer than 1 second. 6 This illuminated area occurs in this probe design and is valid while focusing at the cornea. By focusing in the aqueous humor adjacent to the cornea with $95 \mu \mathrm{W}$ and 1 second $(95 \mu \mathrm{J})$, the exposure is well below this value.

\section{Results}

The chemical structure and the excellent Raman spectra of pure silicone oil in a cuvette are shown in Figure 2. Typical Raman peak positions are located at $488 \mathrm{~cm}^{-1}, 708$ $\mathrm{cm}^{-1}, 1409 \mathrm{~cm}^{-1}, 2906 \mathrm{~cm}^{-1}$ and $2965 \mathrm{~cm}^{-1}$. Because the Raman intensity of the 2906 and $2965 \mathrm{~cm}^{-1}$ peaks are much stronger than the other peaks, these latter two positions were used to identify the silicone oil in vivo. Laser power was $12 \mathrm{~mW}$ with 1 second integration time. 


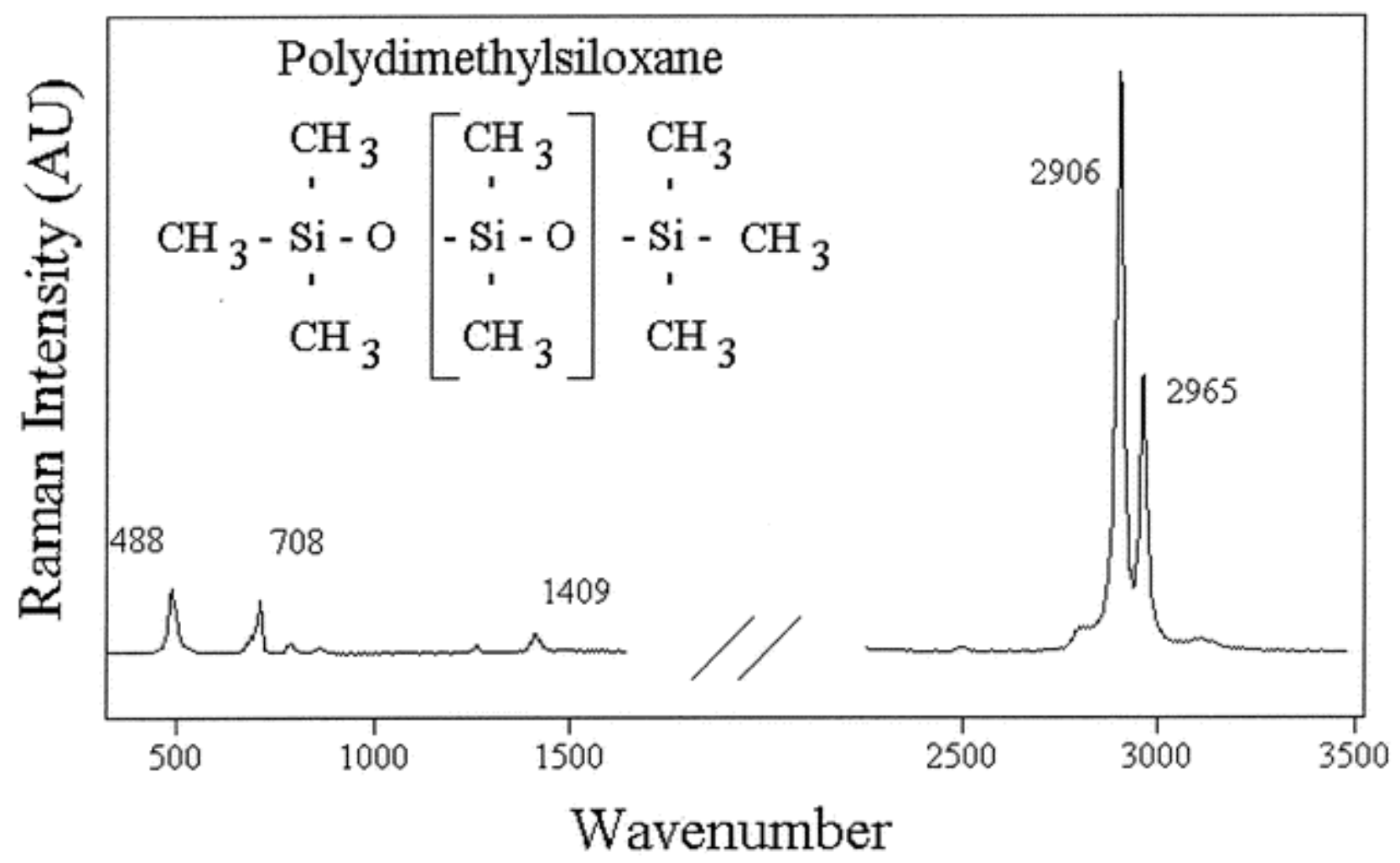

Figure 2. Structure of silicone oil and Raman spectral features.

Figure $3 A$ shows the Raman spectrum of complete silicone oil filling of the anterior chamber in the rabbit. Laser power was $5 \mathrm{~mW}$ and 1 second integration time. Even at this reduced power, the good signal-to-noise ratio provides latitude for the use of less energy. Therefore, we further reduced the power to $0.5 \mathrm{~mW}$ for the next experiment (figure $3 \mathrm{~B}$ ). 

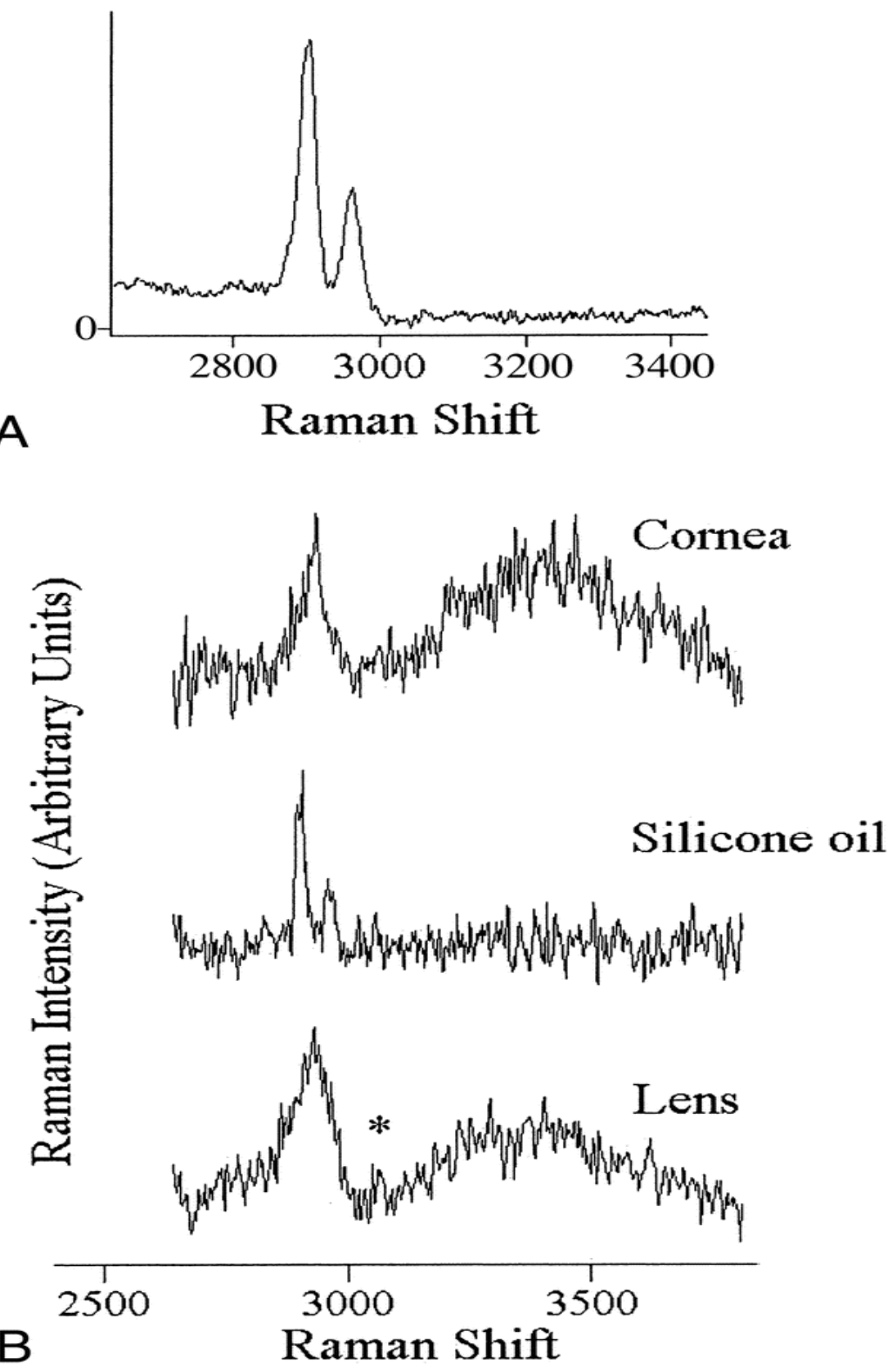

Figure 3. A, In vivo Raman spectrum of the anterior chamber of the rabbit eye injected with silicone oil. $\boldsymbol{B}$, In vivo Raman spectra of the rabbit eye with the confocal probe positioned to observe the cornea, small silicone oil bubble within the aqueous humor, and lens. 
Figure 3B shows Raman spectra of the cornea, a small silicone oil bubble in the anterior chamber, and the lens. The oil bubble, with a diameter of approximately 0.75 $\mathrm{mm}$, was located in the superior anterior chamber. Because of the power reduction and the bubble size, which reduces the integration length, the quality of the signal is somewhat poorer than in figure 3A. The Raman spectrum was obtained by visual focusing on the bubble with the laser beam. The * is the more pronounced position at $3060 \mathrm{~cm}^{-1}$ (Aromatic CH vibration) indicative of lens tissue. As can be seen of the cornea and lens Raman spectra, there is a contribution of water content in these tissues, seen as the more broad spectral feature with an intensity maximum of approximately $3390 \mathrm{~cm}^{-1}$. The silicone oil has no significant contribution of a water signal (even more readily seen in Figure 2), regardless of a laser power of $0.5 \mathrm{~mW}$ and 1 second integration time.

Figure 4 shows a Raman spectrum of silicone oil obtained with a laser power of $95 \mu \mathrm{W}$ and 1 second integration time in the anterior chamber of a patient. The spectrum was obtained through an inferior cornea position (figure 4, insert). Even using this extremely low, eye-safe laser power, a clear Raman spectrum of the silicone oil is observed.

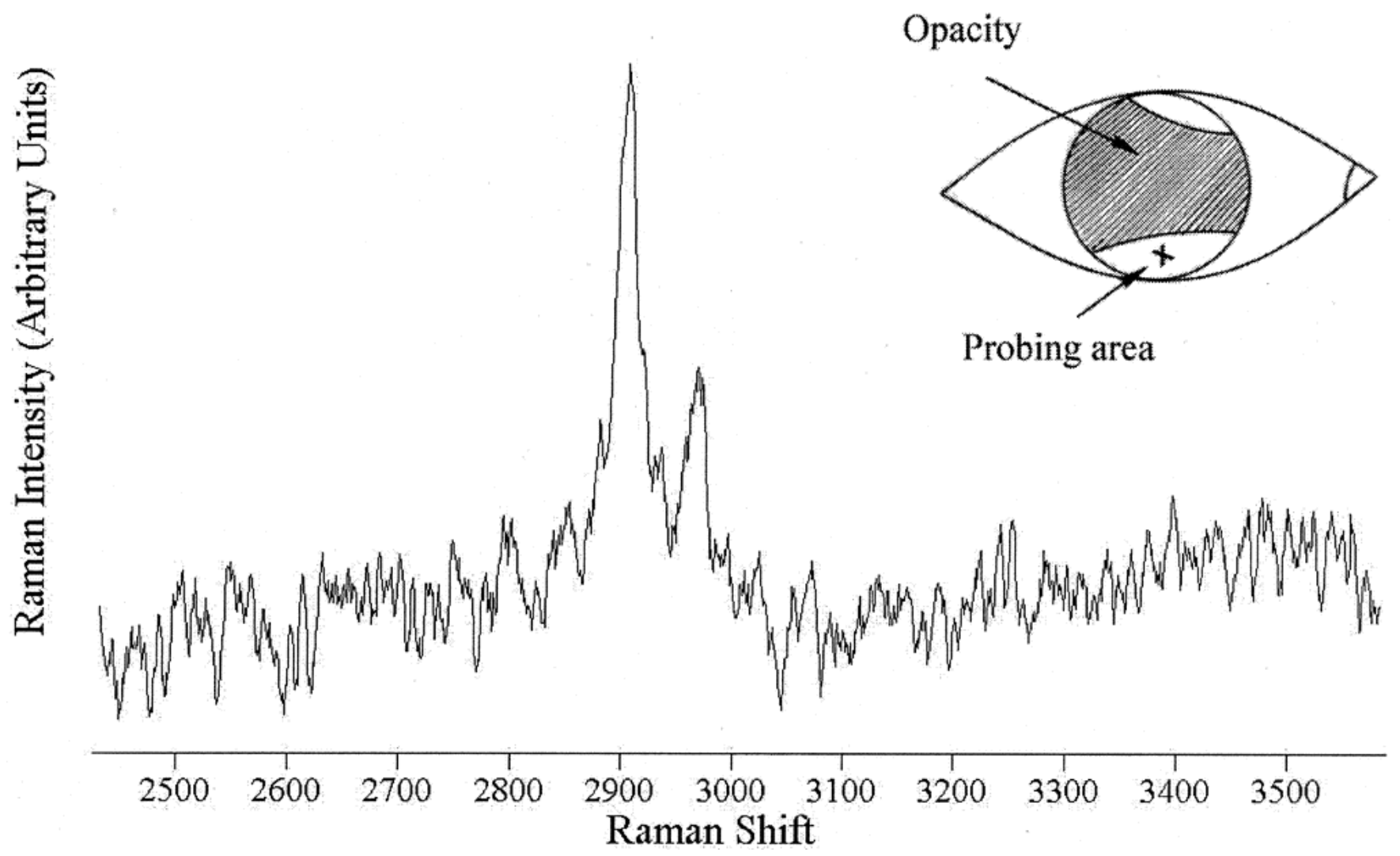

Figure 4. Silicone oil in anterior chamber of a patient. Insert: Human patient eye showing the opaque cornea and probing area for detecting the silicone oil. 


\section{Discussion}

The Raman spectroscopic technique allows for detection and determination of silicone oil in the anterior chamber in the living rabbit eye. The Raman spectra of the cornea, aqueous humor, or lens are clearly distinguished from the introduced silicone oil. The spectrum obtained from the patient shows the feasibility of detection of the silicone oil with a safe laser power in 1 second. The patient had no discomfort during or after the measurement.

In most cases, an experienced ophthalmologist can reliably inspect the eye for silicone oil with the slitlamp biomicroscope. However, to determine the molecular composition of a material, analytical chemistry techniques are required to obtain such information. One analytical technique is Raman spectroscopy, which reveals material characteristics by which a substance's structure can be determined without a priori knowledge about the chemical composition of the substance. Using in vivo Raman spectroscopy, we have introduced another noninvasive technique to detect silicone oil in the eye. The results of the obtained Raman spectra of silicone oil using $95 \mu \mathrm{J}$ are an extension of the results of another study using the current confocal probe setup, where the molecular structure of an implanted intraocular lens (IOL) could be determined in a patient using this safe laser power excitation level ${ }^{8}$.

\section{Conclusion}

Owing to the high specificity and good signal-to-noise ratio of biomaterials, Raman spectroscopy is a promising diagnostic tool to determine the characteristics of these materials. 


\section{References}

1. Allan B. Closer to nature: new biomaterials and tissue engineering in ophthalmology. Review. Br J Ophthalmol 1999; 83: 1235-1240.

2. Ryan S, Ogden T, Hinton D, Schachat A. Retina. St. Louis: Mosby Year Book, 2000 .

3. Erckens RJ, Jongsma FH, Wicksted JP, Hendrikse F, March WF, Motamedi M. Raman spectroscopy in ophthalmology: from experimental tool to applications in vivo. Review. Lasers Med Sci 2001; 16: 236-252.

4. Jongsma F, Erckens R, Wicksted J, et al. Confocal Raman spectroscopy system for noncontact scanning of ocular tissues: an in vitro study. Optical Engineering 1997; 36: 3193-3199.

5. Erckens RJ. Development of the Raman spectroscopic technique for in vivo applications in the eye [thesis]. Maastricht: University of Maastricht, 2001.

6. Bauer NJ. Potential applications of confocal Raman spectroscopy in ophthalmology [thesis]. Maastricht: University of Maastricht, 1999.

7. American National Standards Institute. Safe use of lasers. In: ANSI Standard Z 136.1. Orlando, FL: Laser Institute of America, 1993.

8. Erckens RJ, March WF, Jongsma FH, et al. Noninvasive Raman spectroscopic identification of the intraocular lens material in the living human eye. J Cataract Refract Surg 2001; 27: 1065-1070. 


\section{Chapter 5}

\section{In vivo OCT assessment of rabbit corneal hydration and dehydration}

SPIE proceedings 2003

Keywords: OCT, corneal hydration, corneal thickness, noninvasive monitoring

Alexander I. Kholodnykh ${ }^{1}$, Kamran Hosseini ${ }^{1,4}$, Irina Y. Petrova ${ }^{1}$, Rinat O. Esenaliev ${ }^{1,2,3}$, Massoud Motamedi ${ }^{* 1,4}$

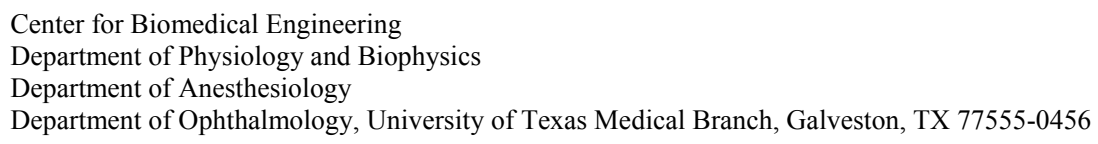




\begin{abstract}
The knowledge of water content of the cornea (hydration level H) can provide crucial information for the assessment of corneal function. The correlation between the corneal thickness and its hydration enables us to estimate $\mathrm{H}$ indirectly by measuring changes in corneal thickness and scattering using OCT. The magnitude and axial distribution of the backscattering signal from the cornea yields additional information about the hydration gradient across the cornea.

We present data regarding the effect of corneal hydration on its thickness and scattering during natural processes of de- and rehydration, as well as in stress tests with the use of glycerol-based dehydrating agent Ophthalgan. Our data demonstrate that scattering signal changes up to 50 times when corneal thickness varies from $60 \%$ to $200 \%$ of its normal state. The distribution of scattering intensity across the cornea also depends on the hydration level and gradient of the water distribution. Thus, OCT can provide a noninvasive and non-contact method for safe and fast measurement of thickness and optical properties of the cornea, and therefore, for estimation of corneal hydration level and corneal function.
\end{abstract}




\section{Introduction}

The goal of our investigation was to measure the scattering of light within the rabbit's cornea with OCT in vivo and monitor its axial distribution depending on the hydration level of the cornea.

Hydration of the cornea harbors an important place within various fields of the physiology and pathology of the human eye. Pathologic conditions such as Fuchs' dystrophy, diabetes and surgical procedures like the popular LASIK surgeries are directly related to the hydration status of the cornea. Clarifying the exact behavior of the water movement across the cornea could: (1) lead to better understanding of how diseases such as Fuchs' endothelial dystrophy and diabetes may alter endothelial function in the cornea, (2) be useful for development and testing of new surgical procedures, and (3) provide methods for rapid assessment of cornea viability in battlefield setting and in cornea transplantation. Hydration correlates strongly with the corneal thickness, and it is the thickness that is measured in the clinic to make estimation about the water content inside this ocular medium ${ }^{1}$. The connection between the hydration $\mathrm{H}$ (mg water per mg dry tissue weight) and corneal thickness $\mathrm{L}$ (in $\mathrm{mm}$ ) for rabbit cornea is described by the following formula ${ }^{2}$ :

$$
H=10 \cdot L-0.42
$$

For average thickness of normal rabbit cornea $L_{0}=370 \mu \mathrm{m}^{3}$ this formula yields $H=3.28$, which correlates well with other authors' data $H=3.04$ for the anterior layers of the rabbit cornea and $H=3.85$ for the posterior ones ${ }^{4}$. On the other hand, it is known that variation in corneal hydration level results in the change of its scattering properties ${ }^{5}$. OCT can register the backscattering signal from the object of interest and its axial distribution with high spatial resolution (10-15 $\mu \mathrm{m}$ in our system). This could give an idea about water distribution across the cornea. Thus, OCT measurements of corneal thickness along with scattering could provide means for assessment of corneal hydration level.

\section{Material and methods}

In our experiments we used white New Zealand rabbits anaesthetized with a mixture of xylazine and ketamine injected intramuscularly. We changed the corneal hydration level in vivo by three methods:

1) We kept the eye open that lowered the hydration of the cornea due to evaporation from the eye surface. We registered the decrease of corneal thickness with the rate of 2.5 $\mu \mathrm{m} / \mathrm{min}$ immediately after the eye was opened, and $0.5 \mu \mathrm{m} / \mathrm{min} 20-40 \mathrm{~min}$ for later time points. The change in thickness in this case was about $15-20 \%$ of its normal magnitude.

2) We kept the eye closed that led to the increase of hydration level due to the absence of evaporation from the eye surface. From time to time we opened the eye for a short period to perform OCT measurements. During this procedure, the corneal thickness was changing with a rate close to $1 \mu \mathrm{m} / \mathrm{min}$, and this increase of hydration reached a level 
above $10 \%$ of the normal thickness about $30-40$ min following the initial closure time of the eye.

3) To extend the range of hydration changes we applied the hypertonic agent Ophthalgan to the surface of the cornea, which is a glycerol-based ophthalmic topical drug clinically used to decompress swollen corneas ${ }^{6}$. This caused the rapid absorption of the water from the corneal surface and its inner volume. Using OCT, we observed a rapid decrease of corneal thickness (by $40 \%$ of its normal magnitude during a period of 3-4 min), and then a sudden increase, which is almost as rapid as the previous shrinkage process (up to the normal thickness for some 5-7 min). Moreover, when the eye was closed, the thickening of the cornea continued for up to 1.5-1.8 times of its normal magnitude. Thus, we managed to change the corneal thickness within the range of $210-680 \mu \mathrm{m}$ that corresponded to the change in corneal hydration level from 1.7 to 6.4 .

We performed the measurements of corneal thickness and its scattering of light with the interferometer-based fiber-optic OCT system, designed at the Institute of Applied Physics (Nizhny Novgorod, Russia), which was described previously ${ }^{7}$.

The accuracy of corneal thickness measurements was 5-10 $\mu \mathrm{m}$ depending on the stability of the eye position from the anaesthetized animal. This accuracy was improved to 2-3 $\mu \mathrm{m}$ when a rubber ring mounted on a stable metal holder was used to immobilize the eyeball and thus to fix the eye position relative to the OCT beam along with the computer post-processing of the results, which allowed the removal of the motion artifacts.

The measurement of scattering signal from different corneal layers (the distribution of scattering within the cornea) is a much more difficult task due to a number of reasons which are outlined as the following:

1) To obtain the real distribution of scattering within the cornea one needs to suppress the speckle noise significantly. Our measurements demonstrated that the standard deviation of speckle noise in the normal cornea could be as much as $5 \mathrm{~dB}$ when the scattering signal stays close within 10-60 dB ranges in logarithmic scale. Thus, to obtain results with an accuracy (standard error of the mean) of scattering signal within 5\% for all levels of scatterings, one needs to reduce the speckle noise to at least $0.5 \mathrm{~dB}$.

In our experiments we used spatial averaging of speckles via lateral scanning of the OCT beam over the surface of the cornea. The scanning area was about $100 \times 100 \mu \mathrm{m}$, determined by the required accuracy improvement ratio and the diameter $d$ of the OCT beam on the corneal surface: $S=(5 \mathrm{~dB} / 0.5 \mathrm{~dB})^{2} \times \pi d^{2} / 4 \approx 10^{4}$, with $d=12 \mu \mathrm{m}$ in our system.

2) When strong focusing of the OCT probing beam is applied (as in our case), the OCT sensitivity is not constant along the corneal thickness, but changes remarkably with the depth (to be more exact, with the change in distance between the focal plane and the site of interest). For a corneal thickness of $0.5 \mathrm{~mm}$, the difference between the scattering signals while the beam is focused on the front (epithelium) or back (endothelium) surface of the cornea can reach $20 \mathrm{~dB}$, which is comparable or even exceeds the signal of scattering itself. Thus, the correction of the signals for this "confocal function" is vital for the cornea due to the inherent low scattering within this ocular medium. Another possibility to avoid the OCT sensitivity dependence is to perform measurements in the plane of exact focusing ${ }^{8}$, shifting it gradually across the cornea. In the present work we 
used both possibilities. Since the attenuation due to scattering is very low in the cornea (we estimated from the extrapolation of the results ${ }^{9}$ to $\lambda=1.3 \mu \mathrm{m} \mu_{\mathrm{t}} \cong 0.03-0.1 \mathrm{~mm}^{-1}$ depending on the hydration level), after the correction for "confocal function", one should be able to acquire the actual distribution of scattering intensity within the cornea.

\section{Results and discussion}

Figure 1 shows the profiles of light scattering within the cornea. Solid line represents the state of normal hydration. The first and most prominent peak corresponds to the reflection from air-cornea interface and shows the position of epithelium covered by tear film. The second peak represents endothelium-aqueous humor interface. The scattering profile in the stroma is in between these two prominent peaks. One can easily see that in the normal state, scattering is much stronger in the front one third of the corneal thickness compared to its other parts. This finding is in agreement with the different anatomical structure of the anterior $1 / 3$ part of the cornea and with the existence of positive water gradient across this ocular structure ${ }^{2}$. Ophthalgan is a hypertonic agent that absorbs water and when we applied it to the surface of the cornea, it caused a remarkable increase in the scattering from the anterior part of the cornea (dashed curve in figure 1). The scattering in the dehydrated layers increased, and the slope of the signal became even steeper. Dotted line in figure 1 represents the rehydrated state of the cornea (eye was closed $5 \mathrm{~min}$ later after Ophthalgan application for $30 \mathrm{~min}$ ). As a result of rehydration, the maximum scattering signal in the anterior part of the cornea dropped about 40-50 times in linear scale compared to the dehydrated state caused by Ophthalgan, and 6-11 times relative to the normal state. As evident, amplitudes of the scattering signals in the posterior part of the cornea are relatively small.

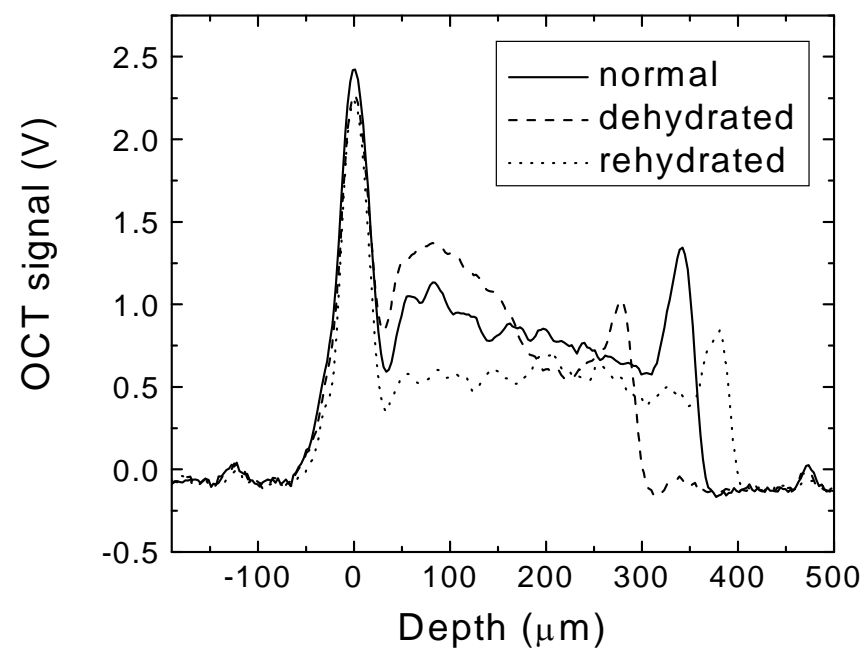

Figure 1. The OCT signals of backscattering from rabbit cornea in vivo corrected for confocal function: solid line - normal level of hydration, dashed line - cornea dehydrated by Ophthalgan, dotted line - rehydrated cornea (eye was closed for $30 \mathrm{~min}$ ). 
Figure 2 demonstrates the dynamics of corneal thickness and backscattering change during in vivo stress test with Ophthalgan.

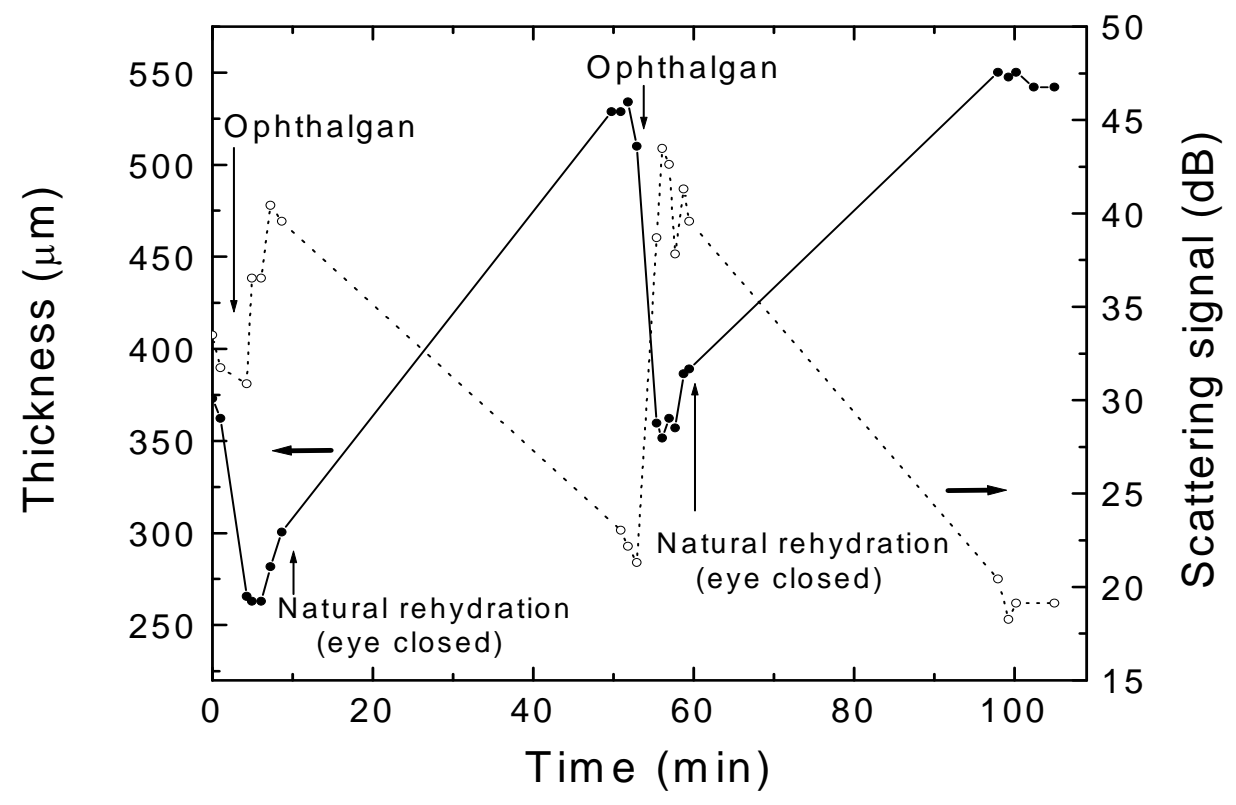

Figure 2. Corneal thickness (solid circles, solid line) and scattering intensity (open circles, dotted line) during dehydration (by Ophthalgan) and rehydration cycles.

Corneal thickness changes up to 2 times between the two extremes of this process, whereas the intensity of backscattered light in the most dehydrated state is about $20 \mathrm{~dB}$ higher (10 times in linear scale) compared to the most hydrated state. The pronounced opposite directions between changes in thickness and scattering is easily seen in these results. This shows that not only from the corneal thickness, but also using the backscattered signal from the stroma one can assess the corneal hydration level. The observed decrease of scattering signal with increasing thickness seems to contradict the results of Maurice ${ }^{5}$, who obtained a 5-fold increase of scattering (instead of decrease in our case) when cornea swelled by 2 times. Perhaps it was due to the fact that Maurice registered the scattering without having the spatial resolution, which is intrinsic to OCT and not in the straight backward direction as it is done in the OCT, but at an angle of $120^{\circ}$ to the direction of incident light.

The comparison of the results obtained in these two manners could yield information on the prevalent change for the number or size of scatterers with corneal swelling. From a medical standpoint, the visible dynamics of scattering in different parts of the cornea as shown in figure 1 could provide crucial information for the refractive 
surgery and cornea transplantation practices. It may also be beneficial in comparing the physiology of the normal cornea to the diseased one and in understanding of the effects of pathological conditions on the cornea's function.

\section{Conclusion}

OCT proves to be a potential device for indirect assessment of water content in the cornea through the measurement of corneal backscattering magnitude. Moreover, the in-depth distribution of backscattering signal can be used for estimation of water gradient across the cornea. 


\section{References}

1. K. A. Polse, R. Brand, S. R. Cohen, M. Guillon, "Hypoxic effects on corneal morphology and function," Investigative Ophthalmology and Visual Science 31, pp. 1542-1552, 1990.

2. B. O. Hedbys and S. Mishima, "The thickness-hydration relationship of the cornea," Experimental Eye Research 5, pp. 221-228, 1966.

3. F. A. Davis, Trans. Amer. Ophth. Soc. 27, p. 401, 1929.

4. R. Turss, J. Friend, M. Reim, and C. H. Dohlman, "Glucose concentration and hydration of the corneal stroma," Ophthalmic Research 2, p. 253, 1971.

5. D. M. Maurice, "The structure and transparency of the cornea," Journal of Physiology 136, pp. 263-286, 1957.

6. M. N. Luxenberg and K. Green, "Reduction of corneal edema with topical hypertonic agents," American Journal of Ophthalmology 71, pp. 847-853, 1971.

7. A. I. Kholodnykh, I. Y. Petrova, K. V. Larin, M. Motamedi, and R. O. Esenaliev, "Optimization of low coherence interferometry for quantitative analysis of tissue optical properties," Proc. SPIE 4624, pp. 36-46, 2002.

8. F. Lexer, C. K. Hitzenbereger, W. Drexler, et al. "Dynamic coherent focus OCT with depth-independent transversal resolution," Journal of Modern Optics 46, pp. 541-553, 1999.

9. R. A. Farrel, R. L. McCally, and P. E. Tatham, "Wave-length dependencies of light scattering in normal and cold swollen rabbit corneas and their structural implications," Journal of Physiology 233, pp. 589-612, 1973. 


\section{Chapter 6}

\section{Accurate in vivo measurements of the corneal light backscattering in-depth distribution with OCT for different hydration states}

SPIE proceedings 2004

Key words: OCT, corneal hydration control, corneal thickness, corneal backscattering, noninvasive monitoring

Alexander I. Kholodnykh ${ }^{1}$, Kamran Hosseini ${ }^{1,4}$, Kirill V.Larin, Alexandra A.Vassilieva, Rinat O. Esenaliev ${ }^{1,2,3}$, Massoud Motamedi ${ }^{1,4}$

Center for Biomedical Engineering

Department of Physiology and Biophysics

Department of Anesthesiology

Department of Ophthalmology University of Texas Medical Branch, Galveston, TX 


\begin{abstract}
On the last conference (BiOS 2003) we presented the corneal light backscattering indepth distribution (CLBDD) as a possible tool for investigation of the corneal hydration control (CHC) and estimation of water gradients within the cornea.

Here, we present new and improved data about the correlation of CLBDD with the magnitude and direction of water gradient within the rabbit cornea in vivo in de- and rehydration processes as well as the relation of the amplitude of light backscattering and the corneal thickness. In contrary to the well-known effect of the immediate increase of the corneal opacity with corneal swelling, we found a new effect namely, an initial decrease of the amplitude of light backscattering in the anterior part of the stroma during the swelling process. The possible explanation for this effect is discussed.

Also methodological improvements for accurate measurements of the CLBDD in vivo are outlined.
\end{abstract}


The main goal of our investigation was to achieve accurate measurement of the corneal light backscattering in vivo using OCT. This offers potentials to investigate not only mean value of the corneal hydration but also the hydration gradients across the cornea, which could result in a better understanding of the corneal pathology and drugcorneal interventions. To achieve the accurate measurement of corneal light backscattering in-depth distribution (CLBDD) with OCT, sufficient speckle-noise suppression is highly recommended. Secondly, it is necessary to suppress the effect of indepth alteration of the OCT sensitivity originated from hard focusing of the probing beam and thus the confocal nature of OCT. The third step is to lower the uncertainties in CLBDD, which arise from possible eye movements during the in vivo measurements. Some of these corrections are already discussed in our previous papers ${ }^{1-3}$.

Previously we have shown ${ }^{2}$ that using averaging technique with a high number (more than $5 * 10^{3}$ ) spatially independent in-depth scans, the uncertainty in the backscattering amplitude measurements can be reduced to a value smaller than $1 \%$.

Unfortunately, achieving such low uncertainty level for the cornea is close to impossible due to its curvature. While performing lateral scanning across the corneal surface, the curvature introduces systematical error in the backscattering amplitude, which increases proportionally with an increase of the scanning area.

Uncertainties due to the curvature and those due to the speckle noise are approximately equal for a lateral scanning area of 100x100 $\mu \mathrm{m}$., which produces uncertainty in the backscattering amplitude of approximately $5 \%$.

In the present work, we managed to decrease this uncertainty down to $2.5 \%$ by using the "image curvature correcting lens" and also by increasing the lateral scanning area up to $200 \times 200 \mu \mathrm{m}$.

In our previous paper", we showed that the "confocal function" is a crucial part of the OCT in-depth sensitivity change although we also have noticed that the confocal function itself is variable within certain limits due to unknown reason(s). In this work we measured the confocal function on the cornea itself. "True" CLBDD for different hydration states corrected for the OCT in-depth sensitivity change are shown in figure 1(a,b,c). The true CLBDD consists an envelope of maximal amplitudes of backscattering signals obtained by consecutive refocusing of the probing beam from the front corneal interface (air/epithelium) to its rear one (aqueous humor/endothelium). 


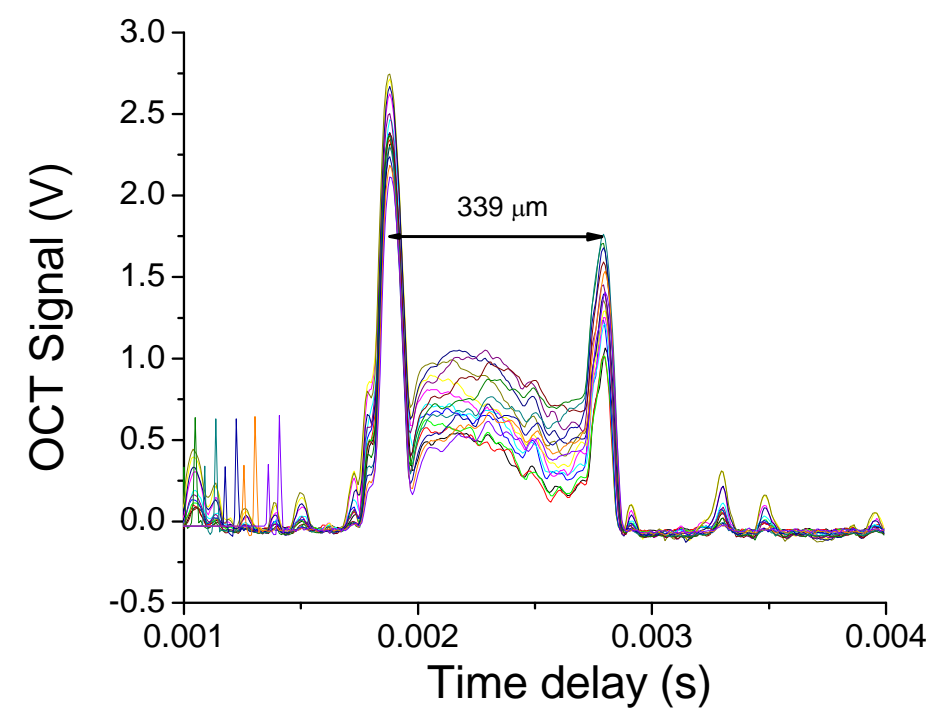

Figure 1a 


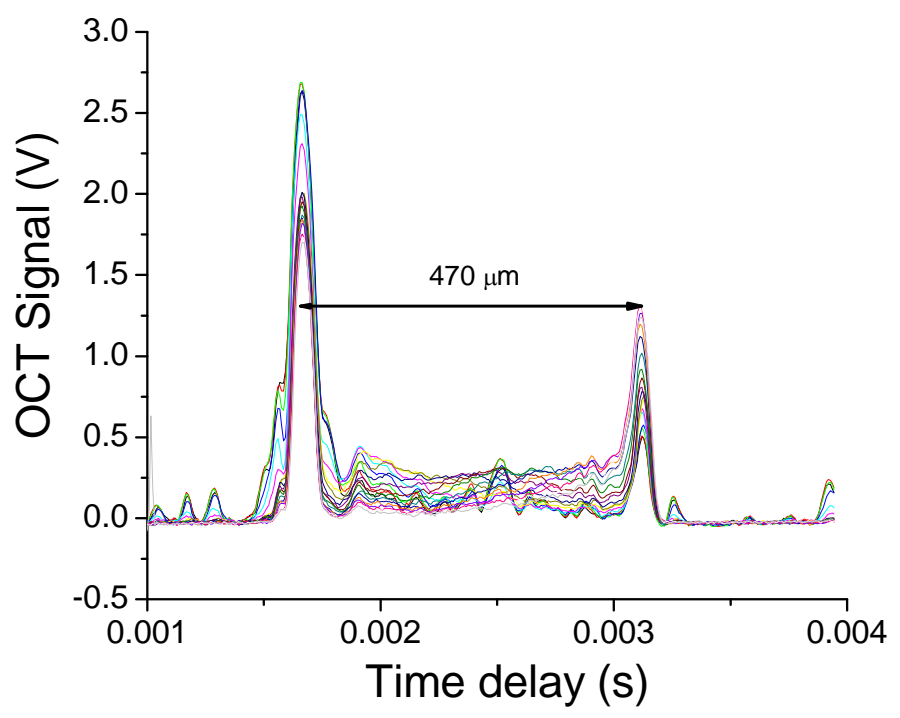

Figure 1b

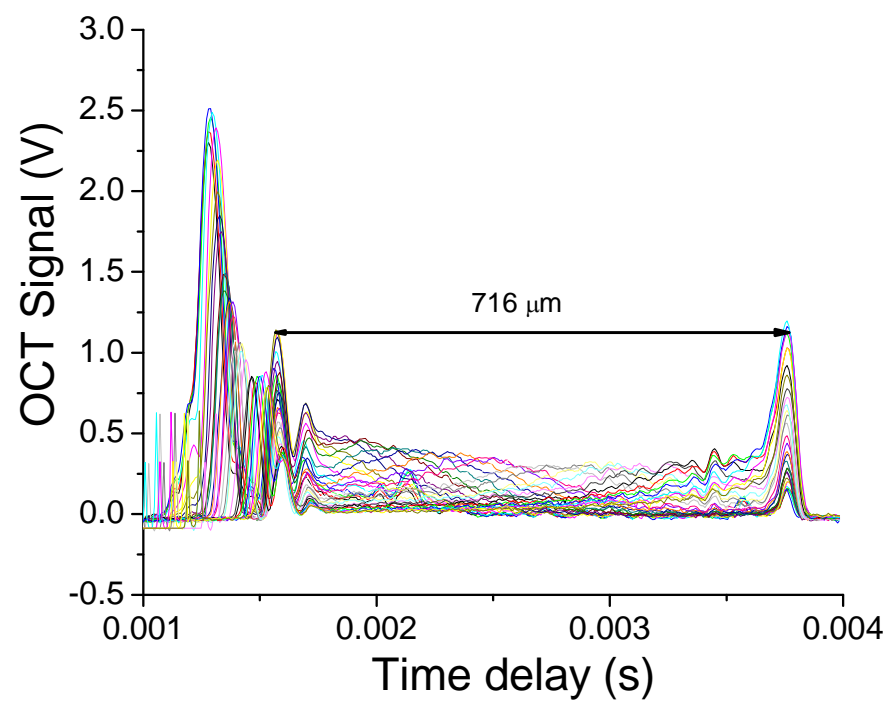

Figure 1c

Figure 1a,b,c. Corneal in-depth distribution light backscattereing for 3 different corneal thicknesses $\left.\left.(L) . a) L=0.92 L_{0}, b\right) L=1.27 L_{0}, c\right) L=1.94 L_{0} \quad$ (normal corneal thickness considered $\left.L_{0}=370 \mu \mathrm{m}\right)$. Figurelc was made while cornea was covered with a layer of water. 
To investigate the effects of the motion artifacts on the backscattering signal, the eye was fixed and the corneal thickness was stabilized around $467 \mu \mathrm{m}$ resulting from steady swelling by covering the cornea under a thin $(10 \mu \mathrm{m})$ transparent film. In spite of the fixation of the eye with respect to the probing beam, the eye showed some small movements, which were mostly due to respiration and heartbeat. In order to correct for these undesirable motions, we additionally performed measurements of the change of the distance between the focal position and the cornea in real-time by monitoring the image displacement on the monitor screen (time delay function). As shown in figure 2, the change in the backscattering signal and the time delay function are "mirror like" functions. This means that the "time delay function" with proper coefficient can be used for simple correction of the backscattering signal. Result of this correction is also shown in this figure. The decrease of the variance in the backscattering signal amplitude is down to a few folds. The efficiency of this method of correction was demonstrated on the estimation of the backscattering signal as a function of the corneal thickness and therefore also as a function of the corneal hydration ${ }^{4}$.

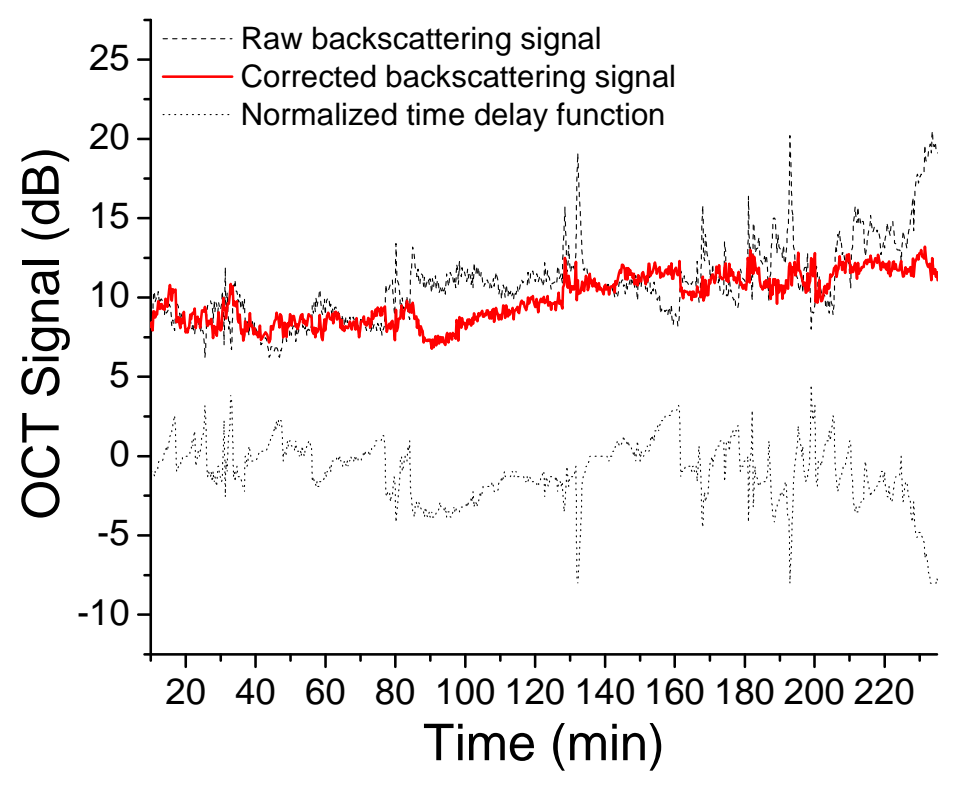

Figure2. Backscattering signal corrected for the eye's motion. 
Results of measurement for the thickness change and backscattering signal as a function of time are shown in Figure 3a. These results were collected during the dehydration process, which was started after maximal swelling was achieved under the covering film. An exponential fit was applied to the results regarding the thickness change. The time constant for this fit proved to be $52.0 \mathrm{~min}$ with a relative uncertainty of only $\pm 0.4 \%$ and $\mathrm{R}^{2}=0.998$. Figure $3 \mathrm{~b}$ depicts the results of corrections for the backscattering signal by using the normalized time delay function as described above.

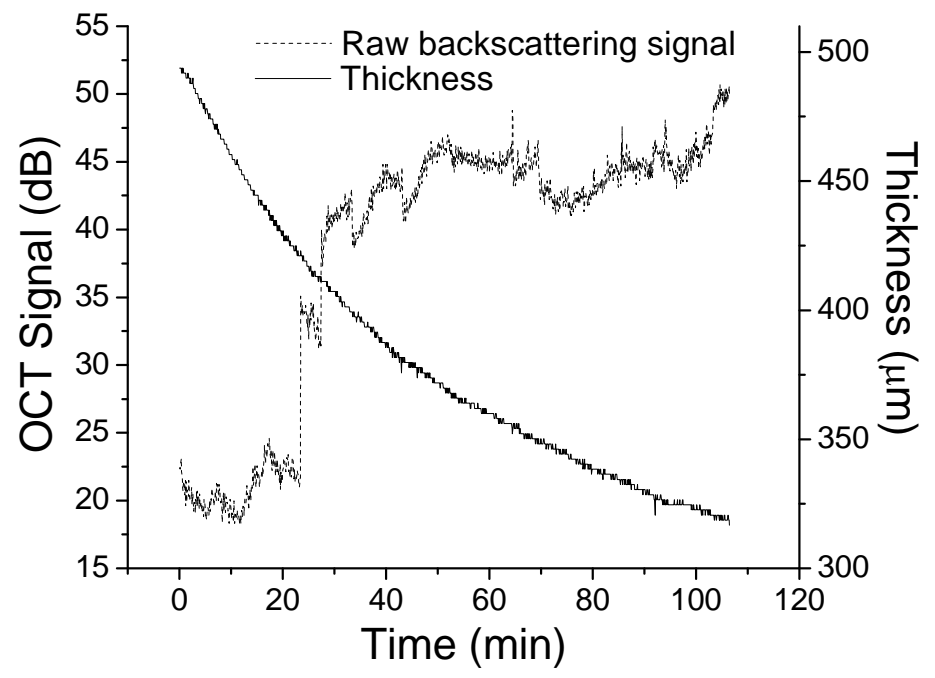

Figure 3a 


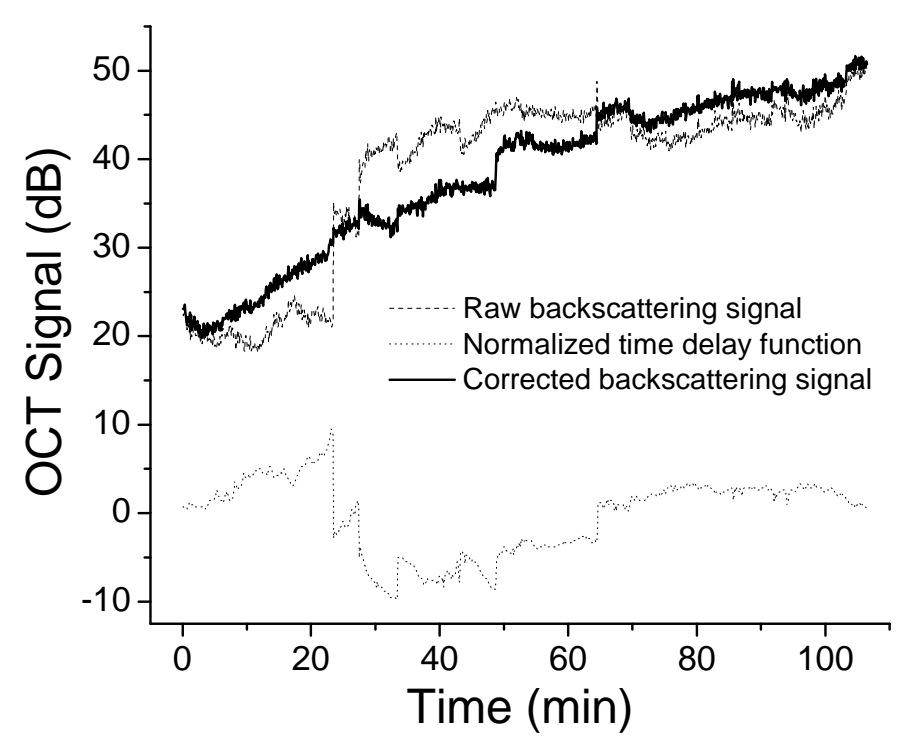

Figure 3b

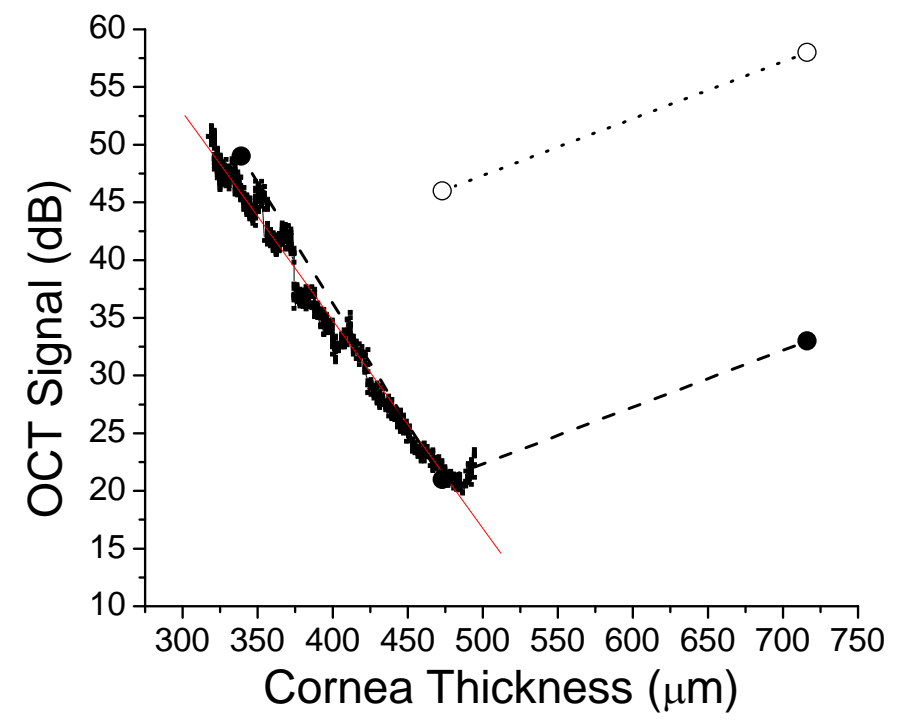

Figure 3c

Figure 3 a)Corneal thickness change and the corresponding backscattering signal as a function of time during the dehydration process; b) Backscattering signal corrected for the eye's motion; c) corrected backscattering signal vs. corneal thickness with approximation for visible spectral range (unfilled dots). 
Using the results presented in figures $3 \mathrm{a}$ and $3 \mathrm{~b}$, we plotted the figure $3 \mathrm{c}$. This figure depicts the corrected backscattering signal versus thickness. The linear fit applied to these results, produces the following relation:

$\mathrm{S}(\mathrm{dB})=(107 \pm 0.3)-(180.3 \pm 0.75) * \mathrm{~L}(\mathrm{~mm})$

(Correlation coefficient $\mathrm{R}=0.989$ and $\mathrm{P}<0.0001$ )

This result is in contradiction with the in vitro study presented by Maurice ${ }^{5}$ who showed that by an increase in the thickness of about $5 \%$, the backscattering would also increase. In our in vivo study, after an increase of close to $30 \%$ in the thickness (maximal swelling achieved in this experiment), there was no increase in backscattering. Results in figure 1 were performed under in vitro condition and therefore they exhibit a bigger range of thickness. After incorporation of those results in figure $3 \mathrm{c}$ ( 3 black filled dots), we can conclude that an increase of the backscattering amplitude could be realized past surpassing the $30 \%$ swelling level. The explanation for this discrepancy can be sought in the fact that we used an infrared source $(\lambda=1.32 \mu \mathrm{m})$ where the backscattering is approximately 20 times smaller than the one for visible light ${ }^{6}$, which was used by Maurice. Using this information we could plot an approximation for the visible spectral range (unfilled dots) in figure $3 \mathrm{c}$. If we consider the discovered decrease of the backscattering signal (figure 3c) wavelength independent, one can connect the approximation line for the visible spectral range with the backscattering signal spreading near the normal corneal thickness value in accordance with results reported by Maurice.

OCT monitoring of the cornea can be utilized for investigation of the water gradient across this structure. To explore that, we investigated two processes, which were opposite of each other in terms of the water gradient. We dehydrated the cornea by exposure of the surface to air at room temperature. We also allowed for rehydration of the cornea by elimination of the surface evaporation by covering it with a plastic film. Typical CLBDD for these processes with an equal corneal thickness (on the whole hydration) are plotted in figure $4 \mathrm{a}$. It is obvious that the anterior part of the stroma in dehydrated state has a different CLBDD profile than its corresponding part in the rehydrated state. This clearly shows a different distribution of the water content across the stromal layers between the two different processes. Figure $4 \mathrm{~b}$ shows a dehydrated cornea, before and 2 minutes after topical application of one drop of artificial tear. The immediate change of the backscattering profile of the anterior stroma is clear with respect to the relative unchanged backscattering profile of the posterior stroma, which shows once more the capability of the OCT monitoring for the water gradient across this ocular structure. Figure $4 \mathrm{c}$ depicts the increase of water gradient due to additional dehydration by forced air evaporation (using a fan) above the free surface evaporation. In opposite to the decrease of backscattering as discussed in figure $4 \mathrm{~b}$, one can observe the increase of the backscattering from the same region of the stroma in figure $4 \mathrm{c}$, as expected. 

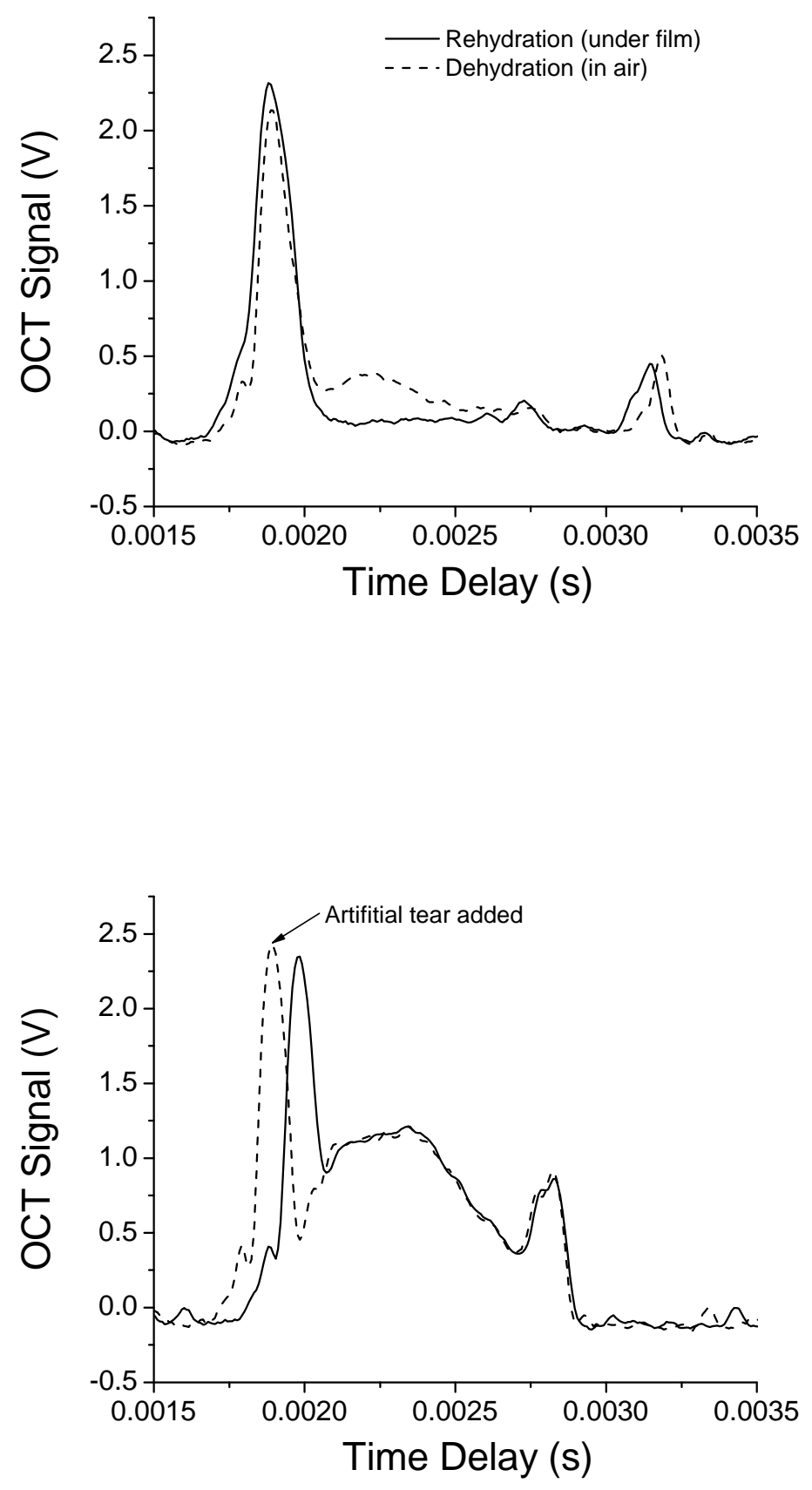


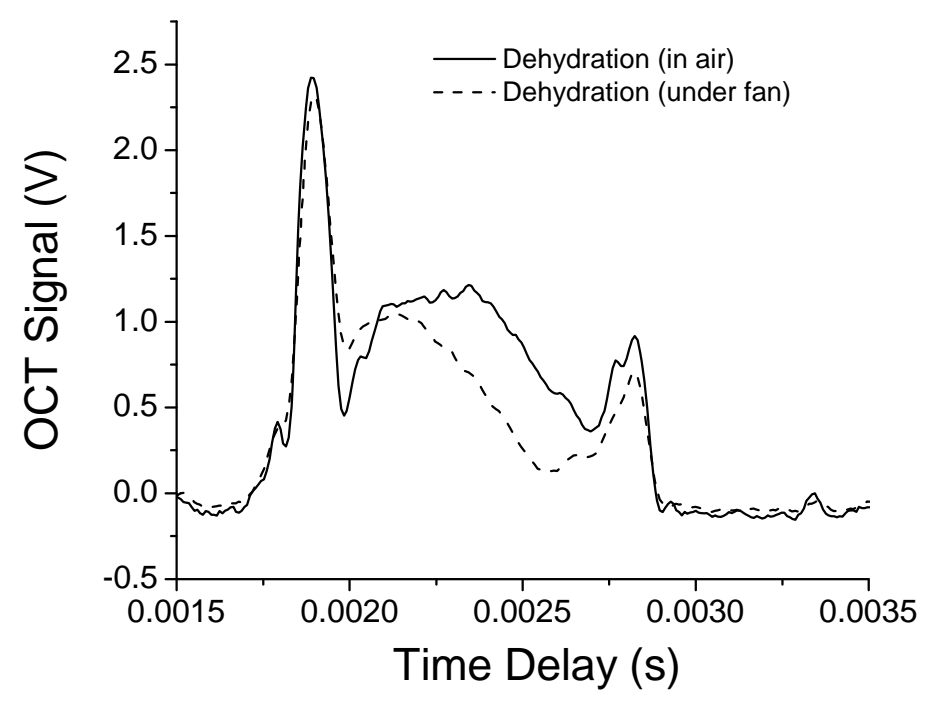

Figure 4. a) Cornea during dehydration (dashed-line) and rehydration (solid-line) processes with approximately equal thickness; b) Dehydrated cornea before (solid-line) and 2 minutes after (dashed-line) topical application of artificial tear; c) Dehydration of the cornea by fan (dashed-line) in comparison with the normal dehydrated cornea (solidline) exposed to air.

\section{Conclusion}

Accurate measurements of CLBDD can be achieved in the cornea, under in vivo conditions using OCT. Furthermore, a relation between the CLBDD and corneal thickness is introduced and a possible explanation for the initial decrease of CLBDD by corneal swelling is offered. Estimation of water gradient across the corneal layers can be concluded by means of CLBDD. 


\section{References}

1. A. Kholodnykh, K. Hosseni, I. Petrova, et al. In vivo OCT assessment of rabbit corneal hydration and dehydration. Proc. SPIE ; Vol. 4956, pp.295-298, (2003).

2. A. Kholodnykh, I. Petrova, K. Larin, M. Motamedi, and R. Esenaliev. Precision of measurement of tissue optical properties with optical coherence tomography. Appl. Opt. Jun 1;42(16):3027-37,(2003).

3. A. Kholodnykh, I. Petrova, Senior Member, IEEE, Massoud Motamedi, and Rinat O.Esenaliev. Accurate measuremant of Total Attenuation Coefficient of Thin Tissue with Optical Coherence Tomography. Accepted on 17 April 2003. Journal of Selected Topics in Quantum Electronics.

4. B. O. Hedbys and S. Mishima, "The thickness-hydration relationship of the cornea," Experimental Eye Research 5, pp.221-228, (1966). D. M. Maurice, "The structure and transparency of the cornea," Journal of Physiology 136, pp.263-286, (1957).

5. D.M. Maurice, "The structure and transparency of the cornea", Journal of Physiology 136, pp. 263-286, (1957).

6. R. A. Farrell, R. L. McCally, and P. E. Tatham, "Wave-length dependencies of light scattering in normal and cold swollen rabbit corneas and their structural implications," Journal of Physiology 233, pp. 589-612, (1973). 


\section{Chapter 7}

\section{Monitoring of Rabbit Cornea Response to Dehydration Stress by Optical Coherence Tomography}

Investigative Ophthalmology and Visual Science. 2004;45:2555-2562

\footnotetext{
Kamran Hosseini $^{1,2,3}$, Alexander I. Kholodnykh ${ }^{1}$, Irina Y. Petrova ${ }^{1}$, Rinat O. Esenaliev $^{1,4,5}$, Fred Hendrikse ${ }^{3}$, and Massoud Motamedi ${ }^{1,2}$

1 Center for Biomedical Engineering, University of Texas Medical Branch, Galveston, Texas

2 Department of Ophthalmology, University of Texas Medical Branch, Galveston, Texas

3 Department of Ophthalmology, University Hospital of Maastricht, Maastricht, The Netherlands

4 Departments of Physiology and Biophysics, University of Texas Medical Branch, Galveston, TX

5 Anesthesiology, University of Texas Medical Branch, Galveston, Texas.
} 


\begin{abstract}
Purpose: To evaluate the application of optical coherence tomography (OCT) for continuous noninvasive monitoring and quantification of the dynamics of corneal response after exposure of the cornea to dehydrating stress.

Methods: The changes in central corneal thickness (CCT) and scattering properties of the cornea were monitored with OCT in rabbit cornea in vivo after topical application of a glycerin-based hypertonic agent (HA) or prolonged surface evaporation of the cornea. The observed changes in backscatter were correlated with the changes in corneal hydration.

Results: An inverse relationship was found between the logarithm of the intensity of backscatter within the cornea and the degree of corneal hydration at which the intensity of the backscatter changed up to 20 times between the peak of the de- and rehydration phases. An analytical relationship is derived between the magnitude of the backscatter from the stroma and the extent of corneal hydration. Furthermore, depending on the concentration of the drug, a peak overshoot in corneal thickness in the range of $40 \%$ to $90 \%$ was detected during the rehydration process after topical application of the HA. At a $100 \%$ concentration of $\mathrm{HA}$, the average dehydration rate was $74 \mu \mathrm{m} / \mathrm{min}$, whereas the average rehydration rate was $12.4 \mu \mathrm{m} / \mathrm{min}$. The same parameters for surface evaporation were 2.7 and $1.5 \mu \mathrm{m} / \mathrm{min}$, respectively.

Conclusions: OCT may offer a unique capability to quantitatively monitor the dynamics of corneal response and to assess corneal function based on noninvasive detection of the changes in the optical properties and morphology of the cornea after topical application of dehydrating agents.
\end{abstract}




\section{Introduction}

Characterization of the mechanism of corneal hydration control $(\mathrm{CHC})$ and the development of new techniques for quantification of water content and its distribution within the cornea have been the focus of many studies ${ }^{1-3}$. Accurate quantitative assessment of corneal hydration or the degree of stress-induced changes in corneal hydration or corneal thickness could have a significant impact on the diagnosis and treatment of various corneal disorders such as Fuch's dystrophy, the precision of the "cut" in refractive surgeries, and cornea transplantation procedures ${ }^{4-6}$. Furthermore, variation in corneal hydration can affect the accuracy of the measurements by diagnostic tools, such as the clinically used intraocular pressure tonometer and the corneal topographer ${ }^{7-9}$. In addition, the effects that drugs may have on corneal hydration can provide valuable information about the function as well as the interaction of drugs with the cornea and the subsequent changes in $\mathrm{CHC}^{10,11}$.

Development of an optical technique for noninvasive monitoring of dehydrationinduced changes in corneal morphology will help elucidate the subtle effects of topical drugs on corneal morphology. Furthermore, this approach could be extended to assess quantitative corneal responses to stress, thus allowing for functional imaging of the cornea.

In the past decade, optical coherence tomography (OCT) has gained great acceptance in the field of ophthalmology because of its high-resolution imaging capability $^{12-15}$. This optical imaging modality produces high-resolution detailed crosssectional images of tissue in vivo and has proven to be very valuable in other medical fields ${ }^{16-18}$.

The unique optical properties of the cornea allow for high-resolution imaging of the entire cornea with OCT. Recent studies have demonstrated the applications of noncontact slit lamp-adapted OCT for in vivo imaging of the human cornea ${ }^{15}$. However, the clinical applications of OCT have primarily focused on high-resolution imaging and qualitative analysis of corneal morphology ${ }^{13,14}$. Recently, we have used OCT for qualitative measurement of the optical properties of the cornea ${ }^{19}$. The strong correlation between corneal thickness and its hydration may promote the use of OCT for quantitative monitoring of corneal hydration in vivo.

The purpose of this study was to demonstrate the feasibility of applying OCTbased techniques for safe, noninvasive, and noncontact monitoring of the dynamics of rabbit corneal dehydration and rehydration in vivo in real time and for the quantitative assessment of rabbit corneal response to dehydration stress based on the measurements of temporal and spatial changes in optical behavior of the cornea. 


\section{METHODS}

\section{Instrumentation}

An interferometer-based fiber-optic OCT system built at the Institute of Applied Physics of the Russian Academy of Sciences ${ }^{18}$ was used in the present study. A superluminescent diode (center wavelength $=1300 \mathrm{~nm}$, incident power on the sample surface $\sim 200 \mu \mathrm{W}$ ) served as a source of low-coherence radiation for the OCT system. Piezoelectric modulation of the fiber length provided in-depth scanning within $2.5 \mathrm{~mm}$. Electromechanical bending of the fiber tip in the focal plane of the objective lens ensured lateral scanning in the adjustable range of 0 to $3 \mathrm{~mm}$. The axial resolution of this system was approximately $14 \mu \mathrm{m}$. In addition to recording OCT images for post-processing, the intensity of light reflected and backscattered from different regions within the cornea was recorded in logarithmic scale by a digital oscilloscope (model TDS 3012; Tektronix Inc., Beaverton, OR). This allowed real-time measurement of the changes in optical properties and morphologic features of the cornea. Further technical descriptions for this system are published elsewhere ${ }^{20,21}$.

\section{Measurements of Corneal Thickness}

Corneal thickness was calculated as the distance between the two major peaks in the oscilloscope signal, which represents reflections from the corneal boundaries (shown with short arrows in figure 1). In all experiments, the corneal thickness was measured at the center of the cornea with a lateral deviation of $\leq 0.5 \mathrm{~mm}$. By focusing the OCT probing beam on the center of the corneal curvature, the variance in corneal thickness is minimized $^{7}$. 
1a

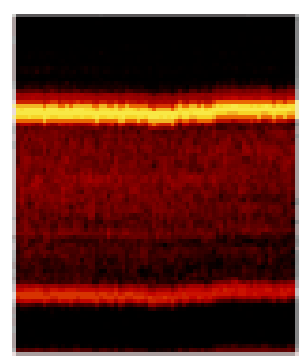

Thickness $(j \mathrm{~m})$

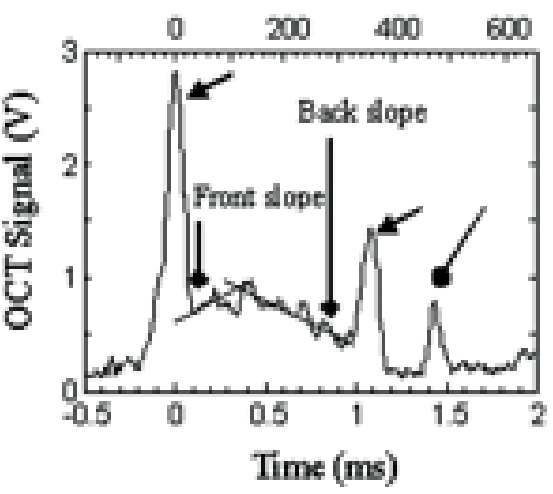

1b

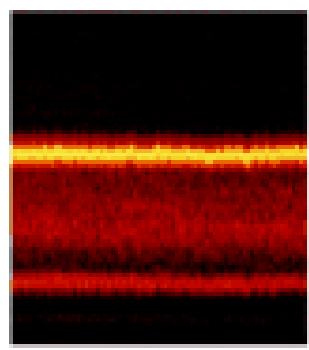

Thickness $(\mu \mathrm{m})$

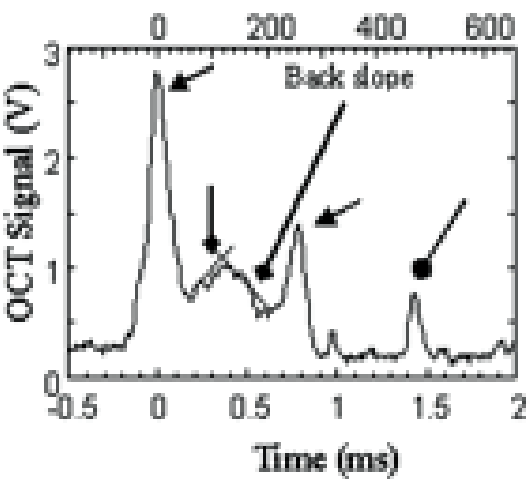

1c
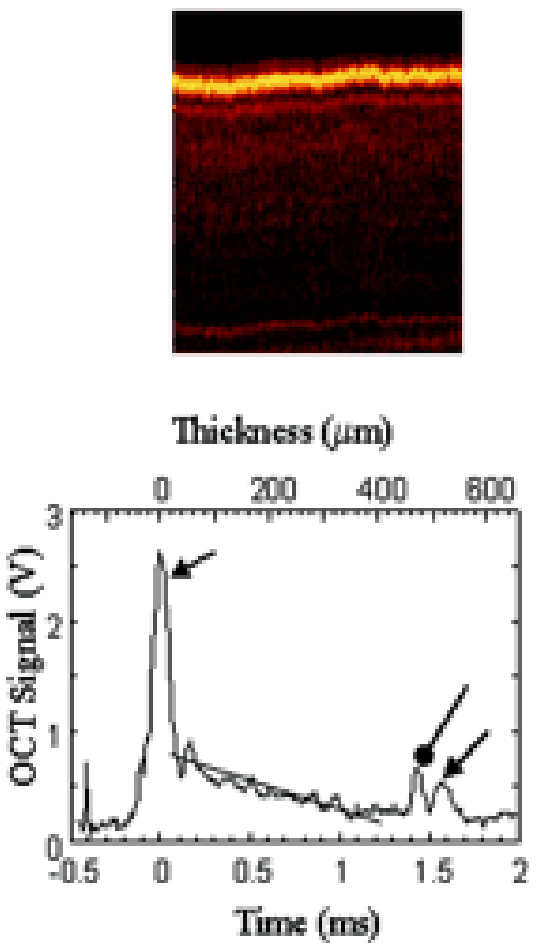

Figure 1. Images of the unperturbed (a), dehydrated (b), and overhydrated (c) states of rabbit cornea in vivo. The corresponding oscilloscope plot is presented below each image. Temporal averaging over 128 scans and spatial averaging over an area of $100 x$ $100 \mu \mathrm{m}$ reduced the noise in the oscilloscope graphs. Short arrows: indicate the reflection peaks from the epithelium and endothelium. Diamond arrows: show front and back slopes. The single oval arrow in each graph shows an artifact caused by internal optical reflections in the OCT system.

The thickness of the cornea was measured based on the measurement of the time delay between the peaks. The coefficient of $0.325 \mu \mathrm{m} / \mu \mathrm{s}$ was used to convert the temporal delay of the oscilloscope measurements into corneal thickness. This coefficient was calculated after measuring the time delay $(2.22 \pm 0.01 \mathrm{~ms})$ between two peaks of reflection on the inner walls of the optical glass cuvette with a known thickness $(1.00 \pm$ $0.01 \mathrm{~mm}$ ) and use of the average corneal refraction coefficient ${ }^{22,23} n=1.38$. We assumed a constant value for this coefficient, independent of the hydration level of the cornea within the range of our corneal hydration manipulation. The precision of the relative peak position reading on the oscilloscope display was $8 \mu \mathrm{s}(3.6 \mu \mathrm{m}$ for air and $2.6 \mu \mathrm{m}$ for the cornea); therefore, the typical thickness of rabbit cornea $(\approx 350 \mu \mathrm{m})$ was measured with an accuracy of better than $99 \%$. Motion artifacts did not affect the accuracy of thickness measurements because a very short time is required to record a single in-depth scan from the cornea $(<0.02$ seconds). 


\section{Measurements of the Backscatter}

Quantification of hydration-induced changes in the scattering properties of the cornea based on OCT measurements requires consideration of several factors that could significantly improve the accuracy of these measurements. The OCT signal produced by scattering in the cornea is $10^{2}$ to $10^{3}$ times smaller than that produced by reflection from the corneal boundaries. This is the reason for using logarithmic amplification of the OCT signal. Moreover, there are speckles in the OCT signal that are a random modulation of the amplitude of the backscatter due to the coherent nature of radiation and interferometric design of systems such as $\mathrm{OCT}^{24,25}$. Recently, we reported that by performing a square pattern scan with the probing beam on the corneal surface $(100 \times 100$ $\mu \mathrm{m})$ and averaging the recorded scans, one can minimize the speckle and electronic noise up to level of a few percents of the OCT signals ${ }^{19}$. Once we reduced the noise in OCT measurements, we were able to quantitatively study the spatial scattering profile within the cornea as a function of time. To prevent artificial alteration of scattering profiles from the changes in the OCT in-depth sensitivity ${ }^{19}$, we focused the OCT probing beam on the epithelial surface of the cornea for all our experiments.

\section{Animal Protocol}

For this study we used white New Zealand rabbits, because their eyes are similar in anatomy and physiology to the human eye. All animal care and treatment procedures adhered to the ARVO Statement for the Use of Animals in Ophthalmic and Vision Research. The rabbits were anesthetized with a mixture of xylazine $(5 \mathrm{mg} / \mathrm{kg})$ and ketamine $(25 \mathrm{mg} / \mathrm{kg})$ injected intramuscularly as needed. The animals were positioned and stabilized, and their eyes were brought into proptosis and held in place with a rubber ring mounted in a fixed, larger metal ring. This holder was designed to position the eye relative to the OCT probe. The ring helped immobilize the eye while at the same time permitting perfusion of the eyeball tissue. An artificial tear was applied as needed to prevent corneal drying before the administration of the stress agent. This approach enabled us to maintain the corneal thickness close to its initial value with a measured variability of approximately 5\%. Ten rabbits were used in the study. One eye of each of four rabbits was subjected to a 100\% hypertonic solution (Ophthalgan; Wyeth, St. Davids, PA; more than $98 \%$ glycerin, $1.0 \%$ water, $0.55 \%$ chlorobutanol) as the dehydrating agent. Another two eyes (two rabbits) were subjected to a 50\% concentration of the same hypertonic solution to investigate the effects of drug concentration on corneal response. Four eyes from a different group of four rabbits were subjected to evaporation at room temperature as another means of dehydration. 


\section{Changing the Hydration State of the Cornea}

Before the hydration manipulations, baseline OCT measurements were performed for 10 to 15 minutes. We then altered the hydration state of the cornea in vivo by topical administration of 1 drop of the hypertonic agent. The strong dehydrating effect of this hypertonic agent caused an almost instantaneous de-swelling of the cornea ${ }^{26}$. The dehydration phase was followed continuously by OCT measurements of the central corneal thickness (CCT) and corneal backscatter. The de-swelling process continued for a couple of minutes and then reached its peak. At that time, the cornea started to rehydrate. As soon as the cornea started to regain some of its thickness and absorb more water, we released the eye from the rubber ring holder. Once the animal's eyelids covered the eye, we taped the eyelids lightly to ensure a relaxed state for the cornea to rehydrate. Then, we opened and examined the eye every 8 to 10 minutes to record the OCT image and oscilloscope signal and to monitor the changes in corneal thickness and the intensity of the backscatter, respectively. The eye was closed again to allow for the rehydration phase to continue. When the rehydration rate was decreased substantially compared to the initial rate of rehydration $(<25 \%)$, we reapplied the hypertonic agent to investigate whether the fact that the cornea that had already been treated with the hypertonic agent would cause any change in its response to the dehydrating agent.

\section{Comparing Dynamics of Different Dehydration}

To compare the influence of different dehydration procedures on the dynamics of the hydration cycles, we performed a similar series of in vivo experiments in which the eye of the rabbit was kept open for an extended period of time to allow for slow dehydration of the cornea at room temperature. Continuous OCT monitoring and oscilloscopic measurements were recorded during the free convective surface evaporation phase of the cornea. Once the corneal dehydration rate diminished substantially, we allowed the cornea to rehydrate in the same manner as with the hypertonic agent-treated rabbits. However, it should be noted that during the rehydration phase of these experiments OCT monitoring was not performed continuously, as was the case in the rabbit treated with the dehydrating agent. At the end of each experiment, the animals recovered fully with no complications.

\section{Data Analysis}

To describe the dynamics of the de- and rehydration processes during the stress test, we applied an exponential fit to our experimental data. This fit has been commonly used to describe the corneal thickness recovery from a swollen state ${ }^{27-31}$. In our experiments, CCT changes take place not only from a swollen state but also from a thinned state, and can reach a new quasi steady state with a different value than the original base thickness. To explain these events, we used an exponential fit in two different forms so that both re- and dehydration processes are addressed independently. 
For the rehydration phase after strong dehydration of the cornea, we used equation 1a :

$$
L(\mathrm{t})=\left(L_{0}-\Delta L_{0}\right)+\left(\Delta L_{0}+\Delta L_{\text {overshoot }}\right)\left[1-\exp \left(-\mathrm{t} / T_{\text {hyd }}\right)\right]
$$

where $\left(L_{0}-\Delta L_{0}\right)$ is the corneal thickness reduced from the base thickness $\left(L_{0}\right)$ at $t=0$ and $\Delta L_{\text {overshoot }}$ is the change in corneal thickness due to overswelling of the cornea (compared with the base thickness $L_{0}$ ) that can be achieved, assuming that the process can reach a quasi steady state. It should be noted that time $t$ can start from any moment during the process that we choose to apply the exponential fit for the experimental data. The parameters of this exponential fit are the time constant $T_{\text {hyd }}$ and magnitude of the CCT change ( $\Delta L_{0}+\Delta L_{\text {overshoot }}$ ). These parameters were calculated through computer software (Origin 7.0 software; Origin Laboratory Corp., Northampton, MA) by curve-fitting the experimental results.

For the dehydration phase from the swollen state we used equation $1 \mathrm{~b}$ :

$$
L(\mathrm{t})=\left(L_{0}+\Delta L_{\text {swel }}\right)-\left(\Delta L_{\text {swel }}+\Delta L_{\text {thin }}\right)\left[1-\exp \left(-\mathrm{t} / T_{\text {deh }}\right)\right]
$$

where $\left(L_{0}+\Delta L_{\text {swel }}\right)$ is the initial thickness $(t=0)$, swelling from the base thickness $L_{0}$, and $\Delta L_{\text {thin }}$ is the magnitude of the decrease in corneal thickness from the base thickness $L_{0}$. The calculated parameters are $T_{\text {deh }}$ and $\left(\Delta L_{\text {swel }}+\Delta L_{\text {thin }}\right)$. (Note: $\Delta L_{\text {thin }}$ is a calculated value in the dehydration process, whereas $\Delta L_{0}$ is a measured value in the rehydration process.)

The initial rate $(d L / d t)$ at time $t=0$ (i.e., maximum due to the exponential nature of the processes) of the CCT alterations can be calculated according to the following formulas:

$$
\begin{aligned}
& d L / d t=\left(\Delta L_{0}+\Delta L_{\text {overshoot }}\right) / T_{\text {hyd }} \\
& d L / d t=\left(\Delta L_{\text {swel }}+\Delta L_{\text {thin }}\right) / T_{\text {dehyd }}
\end{aligned}
$$

To relate drug-induced changes in the corneal hydration, we used a wellestablished analytical relationship between corneal thickness and its hydration ${ }^{32}$ :

$$
H=a \times L-b
$$


where $L$ is the corneal thickness (in millimeters), $H$ is the hydration (milligrams water per milligrams dry tissue weight), and $a$ and $b$ are constants depending on the type of species. In human cornea, for example, $a=7.0$ and $b=0.64$, whereas in New Zealand small rabbits $(<4 \mathrm{~kg}) a=10.0$ and $b=0.42$ have been suggested ${ }^{32}$.

\section{RESULTS}

Figure 1 depicts typical OCT images of the rabbit cornea before induction of dehydration (Fig. 1a), at the peak of dehydration (Fig. 1b), and at the peak of rehydration (Fig. 1c) after topical application of the hypertonic agent.

The corresponding OCT signals monitored by oscilloscope are also provided below the images. The oscilloscope signal in the area between the peaks of reflection from the epithelium and endothelium (marked with short arrows in Fig. 1) represents the backscatter of the stroma, and the larger value of this signal in the dehydrated state corresponds to the brighter bands that appear in the OCT images of the anterior section of the cornea (compare Fig. 1b with 1a and 1c). The considerable decrease in the time delay between the two reflection peaks from the corneal boundaries after application of the hypertonic agent exhibits a proportional decrease of thickness in the corresponding oscilloscope plot shown below this image (Fig. 1b). Also, the increased scattering can easily be detected and quantified in the same plot. The maximum amplitude of the backscatter in Figure 1b is approximately $0.4 \mathrm{~V}$ higher than the amplitude in Figure 1c at the same depth. That means there is a change of approximately $18 \mathrm{~dB}$ in logarithmic scale (which translates to a dramatic increase, up to eight times in linear scale) between the amplitude of the signal corresponding to a totally dehydrated state and that of the overhydrated cornea. There is also a considerable change in the profile of the backscatter in-depth distribution that is dependent on the hydration state of the cornea. The maximum backscatter was found to be located within a $150-\mu \mathrm{m}$ depth below the epithelial layer of the cornea. We introduced two slopes to characterize this profile quantitatively (front and back as depicted by straight lines in Fig. 1). These slopes are steeper in the dehydrated state (Fig. 1b). In Figure 1c, the front slope changes from a positive value to negative value due to overhydration.

Figure 2 exhibits the dehydration and rehydration cycles of rabbit cornea treated with different concentrations (50\% and $100 \%$ ) of the hypertonic agent. A plot of corneal thickness versus time provided a quantitative means to assess the dynamic CCT change during drug-induced dehydration of the cornea. At the same time, the profile of light scattering within the cornea could be visualized in the OCT images. Depending on the state of the hydration of the cornea and the dehydrating procedures used in the experiments, we observed a minimum and maximum CCT of approximately 200 and

$700 \mu \mathrm{m}$, respectively (i.e., a CCT change of up to $500 \mu \mathrm{m}$ caused by the dehydrating effects of the hypertonic agent and the subsequent overrehydration of the treated cornea). 
The two graphs in Figure 2 demonstrate the influence of drug concentration on the rate and extent of dehydration and rehydration of the cornea after topical application of the hypertonic agent. Corneas treated with the $100 \%$ hypertonic agent exhibited an initial rehydration rate of $19 \mu \mathrm{m} / \mathrm{min}$ compared with $10 \mu \mathrm{m} / \mathrm{min}$ for the $50 \%$ hypertonic agent.

As shown in Figure 2, the cornea overshoots its initial thickness during the rehydration process. The cornea treated with 50\% hypertonic agent showed an overshoot of $40 \%$ above its original thickness, whereas the cornea treated with $100 \%$ hypertonic agent concentration exhibited an overshoot of up to $90 \%$ above its initial thickness. For both hypertonic agent concentrations, the temporal responses of the cornea to the first dehydrating stress followed by the second dehydrating stress that was applied after recovery of the cornea from the first stress are depicted in Figure 2. These results show a slower rate of corneal recovery after the second dehydrating stress than that of the cornea's recovery rate after the application of the first dehydrating stress.

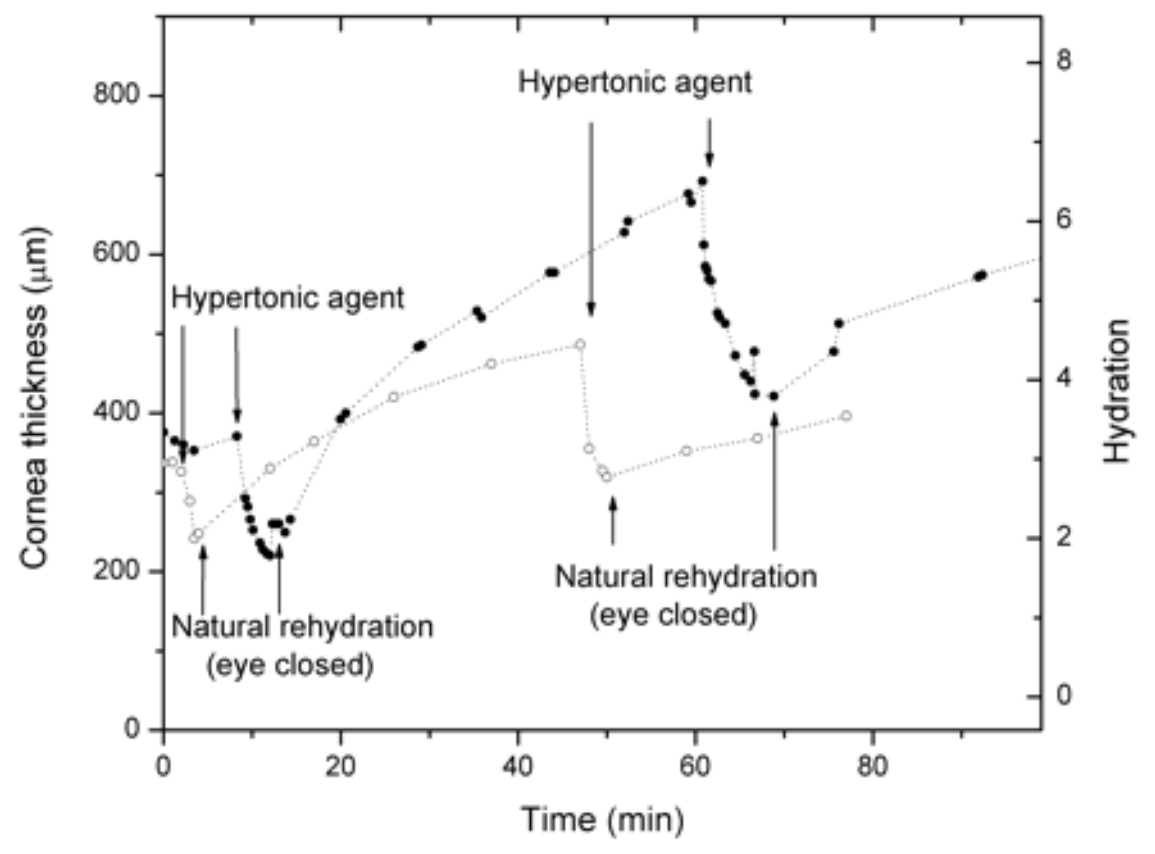

Figure 2. The changes in corneal thicknesses during the de-and rehydration phase of corneal response to topical application of hypertonic agent: $100 \%(\bullet), 50 \%(0)$. The hydration level is indicated on the right y-axis. 
Table 1 presents the data for the CCT recovery in four rabbits after dehydration by the hypertonic agent and shows the range of parameters and accuracy of the fit provided by the proposed exponential model (i.e., equation 1a). These data include the initial corneal thickness, the extent of corneal thinning induced by the application of hypertonic agent (100\% concentration), the experimentally measured overshoot after the stress test, and the statistical parameters of exponential fit. From these data we calculated the average time constant for the recovery process as 23 minutes, with an average initial recovery rate of $12.4 \mu \mathrm{m} / \mathrm{min}$ based on equation $2 \mathrm{a}$.

\begin{tabular}{|c|c|c|c|c|c|c|}
\hline Animal & $\begin{array}{c}\text { Initial } \\
\text { Corneal } \\
\text { Thickness } \\
(\mu \mathrm{m})\end{array}$ & $\begin{array}{l}\text { Induced } \\
\text { Thinning } \\
\quad(\mu \mathrm{m})\end{array}$ & $\begin{array}{l}\text { Experimental } \\
\text { Overshoot } \\
(\mu \mathrm{m})\end{array}$ & $\begin{array}{c}\text { Calculated } \\
\text { Maximum } \\
\text { Overshoot } \\
(\mu \mathrm{m})\end{array}$ & $\begin{array}{c}\text { Calculated } \\
\text { Time } \\
\text { Constant } \\
\left(T_{\text {hyd }}\right) \\
(\text { min })\end{array}$ & $\begin{array}{c}\text { Calculated } \\
\text { Maximum } \\
\text { Recovery } \\
\text { Rate } \\
(d L / d t), \\
\mu \mathrm{m} / \mathrm{min}\end{array}$ \\
\hline 1 & 310 & -124 & 49 & $98 \pm 32$ & $20.4 \pm 7.5$ & $8.0 \pm 3.0$ \\
\hline 2 & 309 & -65 & 37 & $37 \pm 19$ & $14.0 \pm 1.4$ & $7.4 \pm 0.7$ \\
\hline 3 & 319 & -75 & 228 & $322 \pm 38$ & $28.1 \pm 3.2$ & $14.3 \pm 2.0$ \\
\hline 4 & 355 & -156 & 316 & $414 \pm 47$ & $28.8 \pm 3.5$ & $19.8 \pm 1.6$ \\
\hline
\end{tabular}

Table 1. Rehydration (Closed Eye) Subsequent to Dehydration Caused by Application of Hypertonic Agent. *Standard deviation for experimental data is $15 \mu \mathrm{m}$, because of uncertainties caused by application of artificial teardrops prior to the start of the experiments, whereas the relative uncertainty of thickness measurements does not exceed $1 \%$.

The observed rehydration overshoot was reversible over time. Seven days after the dehydration stress studies on the rabbit corneas, we grossly examined the treated corneas and measured their thickness with our OCT system. The corneas had returned to their original thicknesses and showed no sign of edema.

Figure 3 depicts the decrease in corneal thickness during prolonged exposure of the cornea to quiescent air at room temperature. Use of equation $2 \mathrm{~b}$ showed the estimated initial rate of the decrease in corneal thickness to be $2.7 \mu \mathrm{m} / \mathrm{min}$. After the eye was closed, it took approximately 1 hour for the cornea to recover. Thus, the average rate of the recovery process is estimated to be approximately $1.5 \mu \mathrm{m} / \mathrm{min}$. 


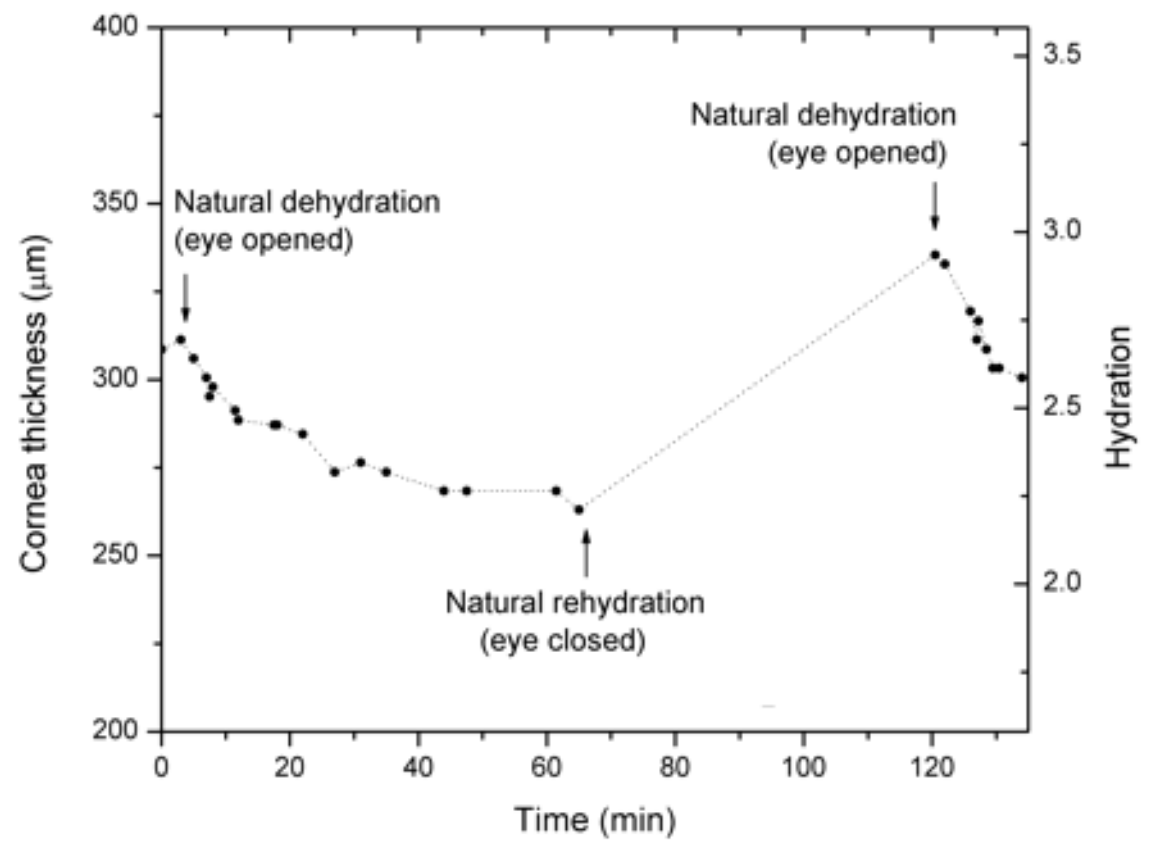

Figure 3. The changes in corneal thicknesses during the de-and rehydration process after corneal exposure to quiescent air as the dehydrating process. The hydration level is indicated on the right y-axis.

Table 2 contains the range, weighted means, and standard deviations of the time constant for the dehydration cycles caused by the $100 \%$ hypertonic agent and the free surface evaporation at room temperature. The average induced thinning was approximately $105 \mu \mathrm{m}$ in the hypertonic agent-treated group. Use of equation $2 \mathrm{~b}$ showed an estimated average rate of $74 \mu \mathrm{m} / \mathrm{min}$ for dehydration-induced changes in CCT. 


\begin{tabular}{|lccc|}
\hline \multicolumn{1}{|c}{ Process } & $\begin{array}{c}\text { Animals } \\
(n)\end{array}$ & $\begin{array}{c}\text { Mean Value of Time } \\
\text { Constant }\left(T_{\text {deh }}\right) \text { Standard } \\
\text { Deviation and Range } \\
(\mathrm{min})\end{array}$ & $\begin{array}{c}\text { Mean Value of Induced } \\
\text { Thinning, Standard } \\
\text { Deviation and Range } \\
(\mu \mathrm{m})\end{array}$ \\
\hline $\begin{array}{l}\text { Hypertonic agent- } \\
\text { induced } \\
\text { dehydration }\end{array}$ & 4 & $1.41 \pm 0.07(0.9-1.8)$ & $105 \pm 39(65-156)$ \\
$\begin{array}{l}\text { Natural } \\
\text { dehydration } \\
\text { (evaporation in air) }\end{array}$ & 4 & $12.5 \pm 0.8(8.7-19.6)$ & $60.6 \pm 3.4(37-89)$ \\
\hline
\end{tabular}

Table 2. The Estimated Time Constants and the Magnitude of the Induced Thinning for Corneal Response during Dehydration Processes for both Hypertonic Agent and Free Surface Evaporation. "Values were calculated as the weighted mean ${ }^{49}$, to take into consideration the change of variance $\sigma^{2}$ (up to two times) for the parameters of the exponential fit within each group: $\bar{X}_{w}=\Sigma\left(X_{i} / \sigma_{i}{ }^{2} \Sigma\left(1 / \sigma_{1}^{2}\right)\right.$.

Figure 4 demonstrates the ability of OCT to provide quantitative details about stress-induced changes in the scattering properties of the cornea. In this study, changes in scattering properties of the stromal tissue during the dehydration and rehydration cycles were monitored. The correlation between changes in corneal thickness and the maximum scattering intensity as a function of time after topical application of the hypertonic agent ( $100 \%$ concentration) is shown in Figure 4. Our analyses show that intensity of the backscattered light varied up to 20 times $(25 \pm 1 \mathrm{~dB})$ between the peaks of the de- and rehydration phases. The uncertainty in the quantification of dehydration- and rehydrationinduced changes in the degree of corneal backscatter is $\pm 1 \mathrm{~dB}$. 


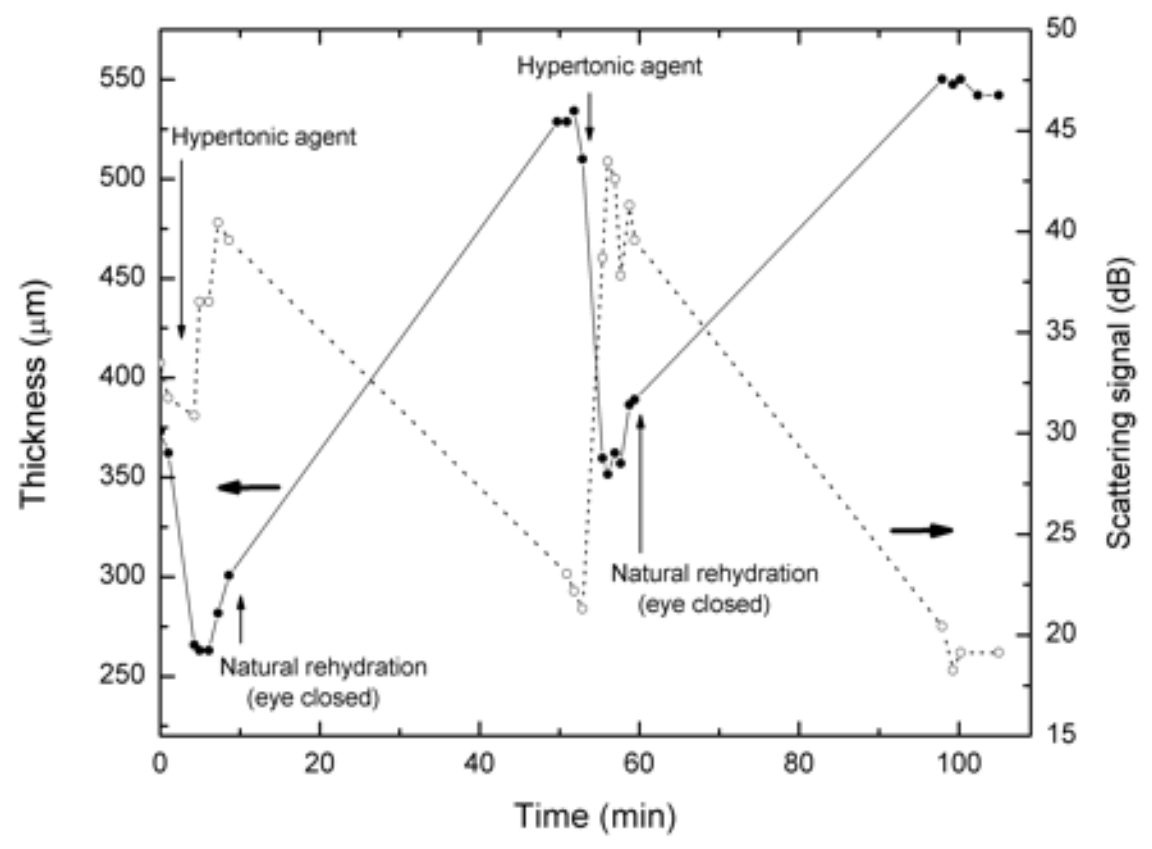

Figure 4. Changes of thickness (•) and backscatter intensity (०) of the cornea as a function of time during dehydration (by 100\% Ophthalgan; Wyeth) and rehydration cycles. Solid and dashed lines: interpolation of experimental data for thickness and corneal backscatter, respectively. 


\section{DISCUSSION}

In the past, many studies have been conducted to investigate $\mathrm{CHC}$ dynamics ${ }^{33-36}$ and the relationship between corneal transparency and the extent of corneal hydration. In most of the studies, corneal swelling is induced by hypoxia, and the rate of corneal deswelling is quantified by measuring corneal thickness as a function of time. There has been considerable interest in developing techniques to quantify the amount and the spatial distribution of water in the cornea. Most of the proposed techniques for direct measurement of corneal hydration require utilization of invasive procedures ${ }^{37,38}$. In recent years, a noninvasive means such as pachymetry ${ }^{39}$ has been developed for clinical use to measure CCT and to infer information about the state of corneal hydration with an indirect approach. However, these measurements often require direct contact of a probe with the cornea and generally do not provide any details about the spatial distribution of water within the cornea. In the present study, corneal thinning was induced, and then the rate of recovery of swelling was investigated while the changes in corneal thickness and backscatter were monitored.

In the past, clinical measurements of light scattering within the cornea have been suggested for the study of corneal hydration and its dynamics ${ }^{40}$. The appearance of bright bands in the OCT image of the anterior part of the cornea in the dehydrated state (Fig. 1b) indicates the qualitative change of the scattering profile across this layer as a result of water movement within the cornea. The local depletion of water within the cornea is known to induce geometric changes between collagen fibrils and to distort the fine hexagonal arrangement between these fibrils that normally ensures the transparency of the cornea ${ }^{23}$. The relatively high amplitude of the backscatter in the anterior one third of the cornea suggests lower water content in this region compared with the other two thirds of the cornea. The change in the hydration state of the cornea also alters the slopes of the backscatter in-depth distribution in the stromal part, as shown in Figure 1, in a reproducible manner. Furthermore, the spatial distribution of the backscatter from the stromal layer correlates inversely with the known water distribution in the cornea, as described by Turss et. $a l^{41}$.

The descending slopes in Figure 2 show the rate of corneal dehydration caused by topical application of two different concentrations of Ophthalgan, the dehydrating agent. It also demonstrates the strong response of the cornea to the dehydration stress by the second ascending slope (rehydration one). This is dependent on the severity of this stress, as evidenced by the speed of recovery of corneal thickness. Comparison of the rate of corneal dehydration after topical application of the hypertonic agent to the rate caused by evaporation on prolonged exposure of the cornea to quiescent air at room temperature (Fig. 3) reveals a significant difference in the speed of the corneal reaction to different dehydrating methods. In the case of drug-induced dehydration, rapid dehydration is followed by a quick rehydration response and an overshoot in corneal hydration, as shown in the thickening of the cornea beyond its initial unperturbed thickness. The magnitude of the overshoot in corneal thickness depends on the amount of dehydrating stress (Fig. 2) for 100\% versus 50\% hypertonic agent concentrations. 
This can also be estimated by observing the disparity in the time constants (Table 2) given for the different dehydrating means. It is interesting to note that an overshoot in human cornea during recovery to its original thickness after a hypoxia stress test was also reported previously ${ }^{34,35,42}$. However, in those reports, the overshoot was captured in the dehydrating process from an edematous (overhydrated) condition caused by induced hypoxia toward the initial unperturbed corneal thickness. In our study, the overshoot occurred in the rehydrating process from a dehydrated state and went beyond the initial corneal thickness.

Figure 4 shows that the intensity of the backscatter correlates inversely with the corneal thickness and hence qualitatively with the hydration of the cornea. From the data presented in Figure 4, a linear relationship between the corneal thickness $L$ (in millimeters) and the magnitude of backscatter $(S$, in decibels) from the cornea in vivo was found by fitting the experimental results depicted in Figure 4 with a linear fit $(R=-0.83$, $P<0.0001)$ :

$$
S(\mathrm{~dB})=(60.3 \pm 4.6)-(71 \pm 10) L(\mathrm{~mm})
$$

Subsequently, by considering equation 3 reported by Hedbys and Mishima ${ }^{32}$ and the observed relationship stated in equation 4 between the magnitude of backscatter $S$ (in decibels) and corneal thickness, one can derive a direct relationship between the magnitude of the backscatter and corneal hydration $(H)$, which can be stated as the following equation:

$$
S(\mathrm{~dB})=(57.3 \pm 5)-(7.1 \pm 1.0) H
$$

This equation can be used for in vivo assessment of the corneal hydration, thus allowing for noninvasive quantitative monitoring of corneal hydration using OCT. From equation 5, one can see that the degree of backscatter light as detected by OCT decreases as cornea hydration increases. To explain this observation, one should consider the lattice structure of the cornea and the diffractive nature of corneal transparency, as originally proposed by Maurice ${ }^{23}$. In the infrared region, during the process of corneal overhydration, the magnitude of the OCT signal is initially influenced by the lattice structure of the cornea. However, as the extent of corneal swelling is increased and the lattice structure of the cornea is disturbed, the wide-angle scattering makes a larger contribution to the detected OCT. Thus, in our view, the observed initial decrease in the OCT signal is primarily due to a swelling-induced decrease in the number of lattice elements within a small volume of tissue that is probed by OCT. 
The observed overshoot can be attributed to the fact that the hypertonic agent used in our experiments can penetrate readily across the cornea, reach the endothelial layer, and affect the pumping action of the endothelial cells ${ }^{43}$. Thus, the direct effects of this hypertonic agent on the endothelial pump function can compromise the function of endothelial cells temporarily. As more water is pulled into the cornea, the observed reversible overhydration and thickening of the cornea after topical application of the hypertonic agent is seen. Although transient reaction of the epithelial layer to glycerin is partly responsible for the observed response of the cornea during the dehydration stress, the response of the cornea to the dehydrating agent is predominantly due to the interaction of glycerin with the stromal tissue and endothelial function. Several cryobiological studies have already investigated the effects of different concentrations of glycerin (the main chemical ingredient of this hypertonic agent) on the cornea and reported findings that support our proposition that the paralyzing effect of the glycerin on endothelial pump function ${ }^{44-48}$ could be responsible for the induction of the overshoot in corneal thickness after the application of hypertonic agent. As mentioned earlier, after the in vivo experiments, the corneas recovered to their original thicknesses and were free of edema 1 week after each experiment. This suggests the reversibility of the observed overshoot and the recovery of the endothelial pump function over time. The ability of this hypertonic agent to induce reversible changes in corneal morphology and optical properties as quantified by OCT monitoring in vivo is also consistent with the findings of other studies that investigated the effects of different concentrations of glycerin on the endothelial layer of the cornea in vitro ${ }^{45,46}$. Collectively, the current in vivo investigation as well as the prior in vitro studies suggests that a stress test using the optimum concentration of dehydrating agent combined with high-resolution OCT measurements can be developed for the quantitative assessment of corneal function and viability.

In addition, it's worth noting that multiwavelength differential OCT measurements can expand the utility of the proposed monitoring technique by mapping the distribution of scatterers and absorbers within the cornea to determine water distribution within an unperturbed cornea.

\section{CONCLUSION}

OCT monitoring could provide a safe, noninvasive, and quantitative method for studying the dynamics of corneal response after topical application of a dehydrating agent. Investigation of corneal backscatter distribution using this technique appears to offer promising prospects for quantitative measurements of the hydration gradient inside the cornea. Thus, quantitative OCT imaging could open a new perspective for noninvasive assessment of corneal morphology, function, and viability. 


\section{ACKNOWLEDGMENTS}

The authors thank Kirill Larin and Brent Bell for technical assistance.

Supported in part by a grant from Research to Prevent Blindness, Inc. 


\section{References}

1. Ruberti JW, Klyce SD. $\mathrm{NaCl}$ osmotic perturbation can modulate hydration control in rabbit cornea. Exp Eye Res. 2003;76:349-359.

2. Pallikaris IG, Ginis HS, Kounis GA, Anglos D, Papazoglou TG, Naoumidis LP. Corneal hydration monitored by laser-induced breakdown spectroscopy. J Refract Surg. 1998;14:655-660.

3. Bauer NJ, Wicksted JP, Jongsma FH, March WF, Hendrikse F, Motamedi M. Noninvasive assessment of the hydration gradient across the cornea using confocal Raman spectroscopy. Invest Ophthalmol Vis Sci. 1998;39:831-835.

4. Kim WS, Jo JM. Corneal hydration affects ablation during laser in situ keratomileusis surgery. Cornea. 2001;20:394-397.

5. Kohlhaas M, Draeger J, Bohm A. Effect of corneal hydration and dehydration of donor tissue on the precision of the thickness in lamellar refractive keratoplasty. Ophthalmologe. 1995;92:410-413.

6. Aboalchamat B, Engelmann K, Bohnke M, Eggli P, Bednarz J. Morphological and functional analysis of immortalized human corneal endothelial cells after transplantation. Exp Eye Res. 1999;69:547-553.

7. Doughty MJ, Zaman ML. Human corneal thickness and its impact on intraocular pressure measures: a review and meta-analysis approach. Surv Ophthalmol. 2000;44:367-408.

8. Simon G, Small RH, Ren Q, Parel JM. Effect of corneal hydration on Goldmann applanation tonometry and corneal topography. Refract Corneal Surg. 1993;9:110-117.

9. Lleo A, Marcos A, Calatayud M, Alonso L, Rahhal SM, Sanchis-Gimeno JA. The relationship between central corneal thickness and Goldmann applanation tonometry. Clin Exp Optom. 2003;86:104-108.

10. Egan CA, Hodge DO, McLaren JW, Bourne WM. Effect of dorzolamide on corneal endothelial function in normal human eyes. Invest Ophthalmol Vis Sci. 1998;39:23-29.

11. Wirtitsch MG, Findl O, Kiss B, Petternel V, Heinzl H, Drexler W. Short-term effect of dorzolamide hydrochloride on central corneal thickness in humans with cornea guttata. Arch Ophthalmol. 2003;121:621-625.

12. Koozekanani D, Boyer K, Roberts C. Retinal thickness measurements from optical coherence tomography using a Markov boundary model. IEEE Trans Med Imaging. 2001;20:900-916. 
13. Neubauer AS, Priglinger SG, Thiel MJ, May CA, Welge-Lussen UC. Sterile structural imaging of donor cornea by optical coherence tomography. Cornea. 2002;21:490-494.

14. Hirano K, Ito Y, Suzuki T, Kojima T, Kachi S, Miyake Y. Optical coherence tomography for the noninvasive evaluation of the cornea. Cornea. 2001;20:281289.

15. Wirbelauer C, Winkler J, Bastian GO, Haberle H, Pham DT. Histopathological correlation of corneal diseases with optical coherence tomography. Graefes Arch Clin Exp Ophthalmol. 2002;240:727-734.

16. Welzel J. Optical coherence tomography in dermatology: a review. Skin Res Technol Rev. 2001;7:1-9.

17. Chan KP, Devaraj B, Yamada M, Inaba H. Coherent detection techniques in optical imaging of tissues. Phys Med Biol. 1997;42:855-867.

18. Roper SN, Moores MD, Gelikonov GV, et al. In vivo detection of experimentally induced cortical dysgenesis in the adult rat neocortex using optical coherence tomography. J Neurosci Methods. 1998;80:91-98.

19. Kholodnykh A, Hosseini K, Petrova I, Essenaliev R, Motamedi M. In vivo OCT assessment of rabbit corneal hydration and dehydration. Proc SPIE. 2003;4956:295-298.

20. Kholodnykh AI, Petrova IY, Larin KV, Motamedi M, Esenaliev RO. Optimization of low coherence interferometry for quantitative analysis of tissue optical properties. Proc SPIE. 2002;4624:36-46.

21. Kholodnykh AI, Petrova IY, Larin KV, Motamedi M, Esenaliev RO. Precision of measurement of tissue optical properties with optical coherence tomography. Appl Opt. 2003;42:3027-3037.

22. Aubert H, Matthiesen L. Graefe-Saemisch Handbuch der gesammten Augenheilkunde. 1876; 1st ed. 409. Engelmann Leipzig, Germany.

23. Maurice DM. The structure and transparency of the cornea. J Physiol. 1957;136:263-286.

24. Goodman JW. Some fundamental properties of speckles. J Opt Soc Am. 1976;66:1145-1150.

25. Schmitt JM. Speckle in optical coherence tomography. J Biomed Optics. 1999;4:95-105.

26. Luxenberg MN, Green K. Reduction of corneal edema with topical hypertonic agents. Am J Ophthalmol. 1971;71:847-853. 
27. Polse KA, Brand R, Mandell R, Vastine D, Demartini D, Flom R. Age differences in corneal hydration control. Invest Ophthalmol Vis Sci. 1989;30:392-399.

28. Mandell RB, Polse KA, Brand RJ, Vastine D, Demartini D, Flom R. Corneal hydration control in Fuchs' dystrophy. Invest Ophthalmol Vis Sci. 1989;30:845852.

29. Herse PR. Corneal hydration control in normal and alloxan-induced diabetic rabbits. Invest Ophthalmol Vis Sci. 1990;31:2205-2213.

30. Weston BC, Bourne WM, Polse KA, Hodge DO. Corneal hydration control in diabetes mellitus. Invest Ophthalmol Vis Sci. 1995;36:586-595.

31. Matsuura T, Gorti S, Tanaka T, Hara Y, Saishin M. Determination of corneal gel dynamics. Eur Biophys J. 1999;28:357-368.

32. Hedbys BO, Mishima S. The thickness-hydration relationship of the cornea. Exp Eys Res. 1966;5:221-228.

33. Maurice DM. The cornea and sclera. Davson H eds. 3rd ed. The Eye. 1984;1b:1158. Academic Press New York.

34. O'Neal MR, Polse KA. In vivo assessment of mechanisms controlling corneal hydration. Invest Ophthalmol Vis Sci. 1985;26:849-856.

35. Bourassa S, Benjamin WJ, Boltz RL. Effect of humidity on the deswelling function of the human cornea. Curr Eye Res. 1991;10:493-500.

36. Siu AW, Herse PR. The effect of age on the edema response of the central and midperipheral cornea. Acta Ophthalmol. 1993;71:57-61.

37. Castoro JA, Bettelheim AA, Bettelheim FA. Water gradients across bovine cornea. Invest Ophthalmol Vis Sci. 1988;29:963-968.

38. Monti D, Chetoni P, Burgalassi S, Najarro M, Saettone MF. Increased corneal hydration induced by potential ocular penetration enhancers: assessment by differential scanning calorimetry (DSC) and by desiccation. Int J Pharm. 2002;31(232)139-147.

39. La Rosa FA, Gross RL, Orengo-Nania S. Central corneal thickness of Caucasians and African Americans in glaucomatous and nonglaucomatous populations. Arch Ophthalmol. 2001;119:23-27.

40. Olsen TH. Light scattering from the human cornea. Invest Ophthalmol Vis Sci. 1982;23:81-86.

41. Turss R, Friend J, Reim M, Dohlman CH. Glucose concentration and hydration of the corneal stroma. Ophthalmic Res. 1971;2:253-260. 
42. Odenthal MTP, Niewuendaal CP, Venema HW, Oosting J, Kok JHC, Kijlstra A. In vivo human corneal hydration control dynamics: a new model. Invest Ophthalmol Vis Sci. 1999;40:312-319.

43. Grass GM, Robinson JR. Mechanisms of corneal drug penetration. I: In vivo and in vitro kinetics. J Pharm Sci. Jan 1988;77:3-14.

44. Bourne WM, Shearer DR, Nelson LR. Human corneal endothelial tolerance to glycerol, dimethylsulfoxide, 1,2-propanediol, and 2,3-butanediol. Cryobiology. 1994;31:1-9.

45. Rich SJ, Armitage WJ. Corneal tolerance of vitrifiable concentrations of glycerol. Cryobiology. 1992;29:153-164.

46. Rich SJ, Armitage WJ. The potential of an equimolar combination of propane1,2-diol and glycerol as a vitrification solution for corneas. Cryobiology. $1991 ; 28: 314-326$.

47. Brunette I, Nelson LR, Bourne WM. Tolerance of human corneal endothelium to glycerol. Cryobiology. 1989;26:513-523.

48. Madden PW. The assessment of endothelial integrity by scanning electron microscopy and fluorescein diacetate staining following treatment with cryoprotective additives. Curr Eye Res. 1989;8:17-36.

49. Taylor JR. An Introduction to Error Analysis. 1997; 2nd ed. 173-179. University Science Books Sausalito, CA. 


\section{Chapter 8}

\section{Noninvasive Monitoring of Water Movement through Rabbit Cornea Using Optical Coherence Tomography}

Keywords - corneal hydration, Water movement, Light scattering, noninvasive monitoring, Optical Coherence Tomography

Kamran Hosseini, ${ }^{1,2,4}$ Alexander I. Kholodnykh, ${ }^{1}$ Sergey Y. Treschev, ${ }^{1}$ Rinat $O$. Esenaliev, ${ }^{1,3}$ Fred Hendrikse, ${ }^{4}$ Massoud Motamedi ${ }^{1,2}$

1 Center for Biomedical Engineering, University of Texas Medical Branch, Galveston, Texas

2 Department of Ophthalmology, University of Texas Medical Branch, Galveston, Texas

3 Departments of Physiology and Biophysics, University of Texas Medical Branch, Galveston, TX

4 Department of Ophthalmology, University Hospital of Maastricht, Maastricht, The Netherlands 


\begin{abstract}
Optical Coherence Tomography (OCT) was used for accurate measurements of corneal in-depth light backscatter distribution (CDLBD) in real-time. Perturbation induced in the in-depth distribution of corneal backscattered light by the transport of fluid across the corneal epithelium can be non-invasively and quantitatively monitored using OCT allowing for the estimation of speed of fluid movement within the cornea and changes in local corneal hydration. In a series of in vivo studies hydrating and dehydrating agents were used to perturb corneal hydration. OCT measurements of changes in CDLBD of rabbit cornea yielded a propagation rate of $1 \mu \mathrm{m}$ per second for hydration or dehydration induced optical perturbation across a rabbit's cornea. Peak changes in local corneal hydration was estimated to increase by approximately $20 \%$ or decrease by $25 \%$ following the topical application of one drop of hypotonic and hypertonic agents, respectively. This means that OCT can be used for in vivo monitoring of dynamical changes in local corneal hydration with a high in-depth resolution.
\end{abstract}




\section{Introduction}

While corneal hydration and its control have been the focus of many studies, noninvasive and noncontact assessment of the hydration dynamics within this sensitive and specialized tissue remain a challenge and even more so under the "in vivo" conditions. A few groups have employed optical techniques to gauge the feasibility of hydration measurement to some extend ${ }^{1,2}$. However, those approaches mostly addressed the static measurement of water content and to our knowledge; no study has been conducted in which water movement across the corneal layers could be monitored both in real time and in vivo, without perturbing the cornea by physical (contact) means. Delicate physiology of the cornea along with its highly dynamic water homeostasis limits its probing by methods that are slow in quantification or require contact for the purpose of measurements. Another challenge is the need for a method that enables monitoring of the water gradient over the whole cross section of the cornea and detecting any subtle changes of this gradient all in real time.

The cornea consists of an epithelial layer covered with tear film from one side and an endothelial layer in contact with the aqueous humor on the other side. Between these two layers, there is stroma, which constitutes about $90 \%$ of the corneal thickness. While the critical role of the endothelial layer to regulate water content of the cornea and thus maintaining the corneal transparency has been recognized by many investigators including the classical work of Maurice ${ }^{10}$, others have shown the importance of additional trans-epithelial water movement influencing the fluid transport within the cornea ${ }^{3,4}$.

As mentioned previously, some investigators have attempted to quantify the degree of the corneal hydration in the past ${ }^{5,6}$. With the exception of Raman Spectroscopy for noninvasive characterization of spatial distribution of water in the cornea ${ }^{1,7}$, most of the other investigated methods are invasive and/or not suitable for clinical applications. Furthermore, Raman Spectroscopy has a disadvantage mainly because of its limitation in measuring one section/layer of the cornea at a time instead of the whole cross section measurement, which is crucial for detection of water transport between the posterior and anterior poles. Thus, the development of an accurate approach for noninvasive and safe assessment of water transport within various layers of the cornea under in vivo condition is still warranted.

Optical coherence tomography (OCT) is among newly developed imaging techniques that have been utilized extensively for high resolution visualization of various ocular tissues including the cornea ${ }^{8,9}$ OCT has also been used for the

precise measurement of the central corneal thickness $(\mathrm{CCT})^{10,11}$. According to the well-known "thickness-hydration" relationship reported by Hedbys and Mishima $^{12}$, OCT measurements of the CCT can be used as an indirect measurement of the overall hydration of the cornea. However, measurement of the corneal thickness provides no specific information regarding water movement or the spatial distribution of water within the cornea. OCT could be used as a non- 
invasive tool to quantitatively monitor light backscattering within the cornea. In our previous studies ${ }^{13,14}$, we used OCT to monitor total changes in the corneal indepth light backscattering distribution (CDLBD) as a function of corneal hydration. These studies demonstrated a strong correlation between the degree of changes in light backscattering within the cornea and the changes in corneal thickness. Considering these observations and the well-known "thicknesshydration" relationship for the cornea, it may be possible to use OCT for noninvasive monitoring of hydration-induced changes in CDLBD as a function of time and utilize the hydration-induced changes in the degree of backscattering in cornea to quantitatively assess the spatial and temporal distribution of water within the cornea.

In the current study, we performed in vivo studies to demonstrate the ability of OCT to quantitatively monitor the hydration-induced changes in CDLBDs enabling visualization of water movement within the rabbit cornea following topical applications of either hypotonic or hypertonic agents.

\section{Materials and Methods}

\subsection{Animal protocol}

Animal studies were performed in White New Zealand rabbits. All animal care and treatments were performed in compliance with the ARVO statement for the use of animals in ophthalmic and vision research. A mixture of Xylazine (5 $\mathrm{mg} / \mathrm{kg})$ and Ketamine $(25 \mathrm{mg} / \mathrm{kg})$ administered intramuscularly was used as the anesthetic agent. The animals were positioned and stabilized, and their eyes were brought into proptosis and held in place with a rubber ring mounted in a fixed metal ring designed to immobilize the eye during OCT imaging ${ }^{13}$.

\subsection{Instrumentation and calibration}

A custom-made interferometer-based fiber-optic OCT system was used in this study ${ }^{15,16}$. The source of low-coherent radiation for OCT system was a superluminescent diode with a center wavelength set at $1.3 \mu \mathrm{m}$ and an output power of $\sim 200 \mu \mathrm{W}$ delivered to the tissue. The axial resolution of the system was about $15 \mu \mathrm{m}$ while the lateral one was about $14 \mu \mathrm{m}^{16}$. In-depth scanning (A-scans) within $2.5 \mathrm{~mm}$ could be performed with a frequency of $57 \mathrm{~Hz}$ using this system. Also, the system was engineered to allow lateral scanning (B-scans) of probing beam within a range of $1 \times 1 \mathrm{~mm}^{2}$. Average time for data acquisition of $300 \mathrm{~A}$ scans and acquisition of a 2-D image (300x300 pixels) was about 5 seconds. A digital oscilloscope (Tektronix TDS 3012, Tektronix Inc. Beaverton, OR, USA) was additionally used for real-time measurements and calibration of the OCT signals, where the signals were proportional to logarithm of the light intensity back reflected or backscattered from the corresponding depths of the cornea. OCT signal was measured in Volts, where every $0.46 \mathrm{~V}$ of signal change as measured by oscilloscope corresponded to a 10 times change in the intensity of 
backscattered light. CDLBD profiles were extracted from 2-D images using specially developed software.

It is well known that quantitative analysis of the tissue backscattering amplitudes requires suppression of speckle noise present in OCT images. This was accomplished by obtaining two-dimensional B-scans by the OCT probing beam and averaging the results as described previuosly ${ }^{16-18}$. In the current study, the probing beam was focused on the corneal epithelium surface and laterally scanned area of the cornea was limited to less than $200 \times 200 \mu \mathrm{m}^{2}$ in order to prevent signal distortion that may be induced by the corneal curvature due to the effect of the so-called OCT confocal function ${ }^{19,20}$ (dependence of the OCT sensitivity on the distance from the focal plane of the focused probing beam to the probed sample site). Applying the scanning beam orthogonal to the corneal surface minimized this signal distortion. Random eye movement can also cause signal distortion. To minimize the influence of this distortion on the accuracy of the measurements, we monitored the (peak amplitude-) stability of the air/cornea back reflected peak time delay (i.e. stability of the OCT interferometer sample arm length) using oscilloscope.

The confocal function of the OCT system was characterized directly for the rabbit cornea under in vivo conditions. This was achieved by obtaining stepwise measurements of the magnitude of corneal backscattering across its layers. For these measurements, the focusing objective was shifted about (40-50) $\mu \mathrm{m}$ at each step towards the endothelium of the cornea starting from the epithelium. Due to the differences in the refractive index of the cornea and that of the air, the focal plane of the probing beam shifted approximately (55-70) $\mu \mathrm{m}$ at each step within the cornea. This means that after 5-6 steps, the entire rabbit cornea was scanned axially. Subsequently, 5-6 backscattering profiles were obtained for each cornea. The "real" backscattering profile of the cornea was then successfully reconstructed using different components of the corneal backscattering profiles obtained from various focal planes. To establish the capability of OCT to quantitatively monitor backscattering changes in corneal tissue and measure the degree of uncertainties associated with backscattering amplitude, we compared the in-depth profile of the backscattered light from the rabbit cornea in vivo to measurements performed on a cornea phantom made from a plastic film (with known optical properties and with dimensions and curvature similar to those for rabbit cornea). 
2.3 Instrumentation Corneal hydration manipulation and measurement

In order to induce water movement within the anterior region of the cornea, the rabbit cornea was dehydrated or rehydrated by topical applications of one drop $(\sim 6 \mu \mathrm{L})$ of dextrose $(50 \%)$ or pure water, respectively. Each eye was subjected only once to either form of the hydration manipulation means. These experiments were performed in eight eyes.

The estimation of the speed of fluid movement within the cornea and local change of the corneal hydration is based on two reported relationships between the physical properties of the cornea and its hydration. The first one is the wellknown relationship between the corneal thickness and its hydration ${ }^{12}$ :

$$
\mathrm{H}=\mathrm{a} \cdot \mathrm{L}-\mathrm{b} \text {, }
$$

where $\mathrm{H}$ is the degree of hydration (milligrams of water per milligrams of dry tissue weight), $\mathrm{L}$ is the central corneal thickness in $\mathrm{mm}$, and $\mathrm{a}$ and $\mathrm{b}$ are constants depending on the type of species.

The second one is the relation between the corneal thickness and logarithm of the corneal backscattering measured by OCT as we reported previously $^{13,14}$ :

$$
\mathrm{S}=\mathrm{A}-\mathrm{B} \cdot \mathrm{L} \text {, }
$$

where $\mathrm{S}$ is the peak of backscattering distribution in CDLBD and $\mathrm{A}$ and $\mathrm{B}$ are constants. Significance of this relation (2) was demonstrated by the presence of high correlation coefficient for the linear fit that was obtained for the experimental results $\left(\mathrm{R}=0.83^{13}, \mathrm{R}=0.99^{14}\right)$ with $\mathrm{P}<0.0001$ in spite of the presence of significant variations in A and $\mathrm{B}$ (up to 2 times) for different rabbit corneas. For small hydration-induced changes in the corneal thickness $(\Delta \mathrm{L} / \mathrm{L}<<1)$, relations (1) and (2) can be restated as the following:

$$
\begin{aligned}
& \Delta \mathrm{H} / \mathrm{H} 0=1.12 \cdot \Delta \mathrm{L} / \mathrm{L} 0 \\
& \Delta \mathrm{S} / \mathrm{S} 0=-(1.3 \pm 0.5) \cdot \Delta \mathrm{L} / \mathrm{L} 0
\end{aligned}
$$

where $\mathrm{H}_{0}, \mathrm{~L}_{0}, \mathrm{~S}_{0}$ are the corneal hydration, thickness and maximal backscattering signal for a normally hydrated cornea, respectively. In the relations (3) and (4), the numerical values that were reported previously for the constants $a, b, A$, and $\mathrm{B}^{12-14}$ were used.

By combining (3) and (4), one can derive the following direct relation between $\Delta \mathrm{H} / \mathrm{H}_{0}$ and $\Delta \mathrm{S} / \mathrm{S}_{0}$ :

$$
\Delta \mathrm{H} / \mathrm{H} 0 \cong-(0.9 \pm 0.3) \Delta \mathrm{S} / \mathrm{S} 0
$$

As suggested by relation (5) the relative change of the mean corneal hydration is simply proportional to the relative change of the maximum of the corneal backscattering signal. It also means that the intensity of corneal backscattering changes exponentially as function of the corneal hydration.

It should be noted that relations (1) and (2) and consequently (5) were experimentally derived for the steady state corneal conditions. However, in the current study, we are using relation (5) as a first order approximation to model 
water movement in the cornea by characterizing the temporal and spatial changes in the corneal backscattering.

\section{Results}

3.1 Methodological results

Figure 1 demonstrates the crucial role of the speckle noise suppression to obtain a "true" CDLBD profile void of artifacts. In Figures $1 \mathrm{~A}$ and $1 \mathrm{C}$, the in-depth backscattering distributions that were recorded from the homogeneously scattering phantom and rabbit cornea in vivo without performing any spatial Bscans are shown. While the most prominent peaks depicted in A scan represent the back reflection from the anterior and the posterior interface of the corneal and phantom, the profiles of backscattering intensity located between these prominent peaks look like those for a non-homogeneously scattering media. In Figures 1B and 1D, the same profiles are shown again but this time they were recorded with two-dimensional B-scans over an area equal to $100 \times 200 \mu \mathrm{m} 2$ Using spatial averaging technique, the speckle noise in Figures 1B and 1D were successfully suppressed. It should be noted that the backscattering distribution in Figure 1B resemble the classical profile of a homogeneously scattering media. This means that a non-uniform backscattering profile across the cornea as observed in Figure $1 \mathrm{D}$ is caused by the unique structure of the cornea and it is not due to artifacts. According to Figure $1 \mathrm{~B}$, the uncertainty in the amplitudes of the backscattering signals $\mathrm{S}$ can be estimated as $\Delta \mathrm{S} \leq \pm 0.01 \mathrm{~V}$. For corneal measurements where amplitude of the backscattering signal measures as $\mathrm{S} \approx 1 \mathrm{~V}$ (see Figure $1 \mathrm{D}$ ), the relative uncertainty can be estimated as $\Delta \mathrm{S} / \mathrm{S} \leq 1 \%$ assuming there are no systematical errors and instrumental artifacts. 

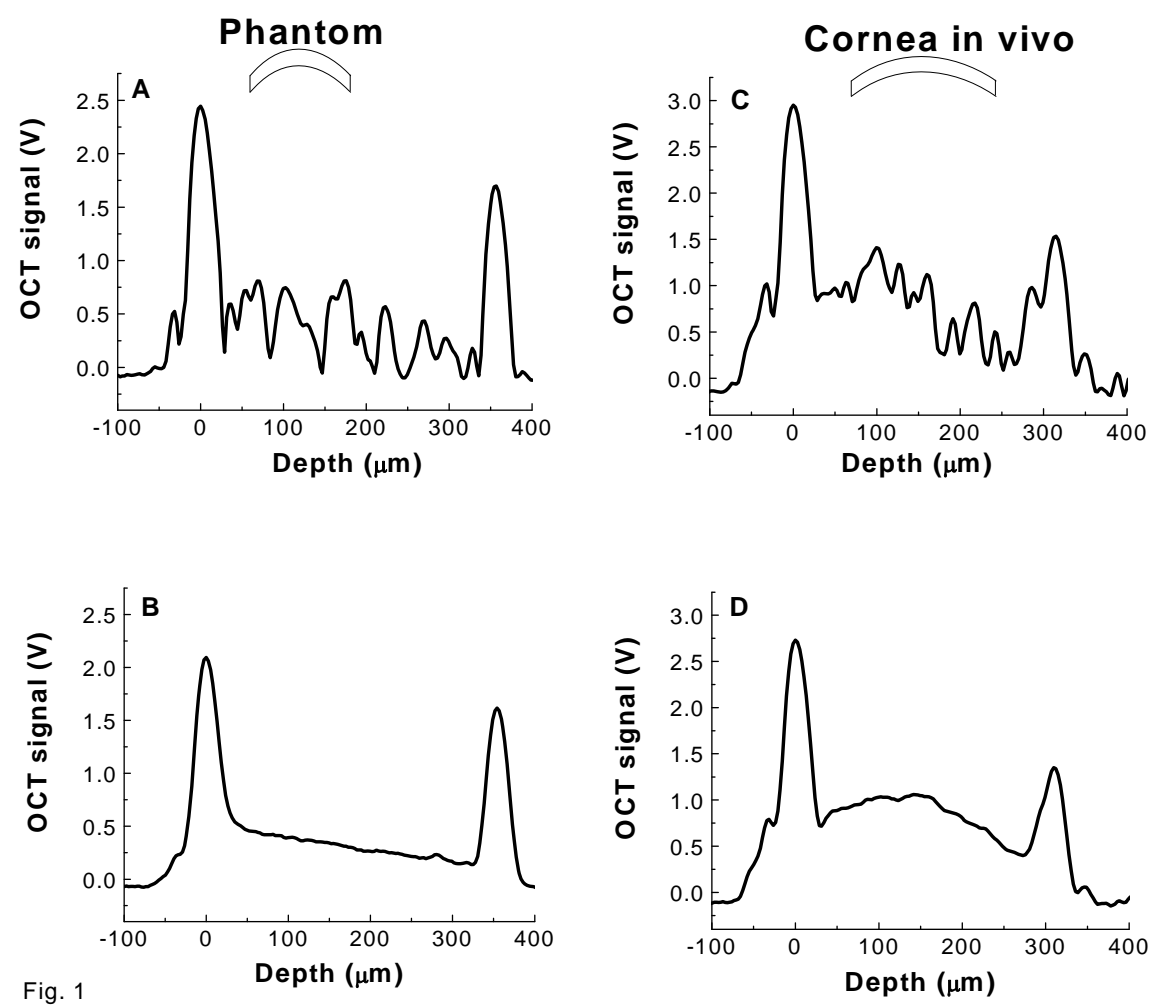

Figure 1. The effect of spatial scanning and averaging on the backscattering profiles for a phantom with homogeneous backscattering and a rabbit cornea in vivo. (A) Typical profiles of the backscattering signal for the phantom cornea without spatial scanning (only temporal averaging); (B) The same backscattering profile with spatial scanning and averaging; (C) Typical profiles of the backscattering signal for the cornea without spatial scanning (only temporal averaging); (D) The same backscattering profile with spatial scanning and averaging; (temporal averaging - over 512 in-depth scans; spatial scanning over area $\left.100 \times 200 \mathrm{\mu m}^{2}\right)$.

For accurate measurement of CDLBD we must also take into account the contributions of systematic errors that may be present and are mainly caused by the depth dependence of OCT sensitivity (so called "OCT confocal function"). Figure 2A illustrates the significance of the OCT confocal function and its influence on the profile of backscattering distribution within the rabbit cornea. The first backscattering profile (bold solid line) was acquired while the OCT probing beam was focused on the epithelium, whereas the sixth profile (dotted line) was collected after moving the focusing objective forward in five consecutive steps resulting in focusing the beam directly on the endothelial layer by the last scan. In Figure 2A, it can be observed from the amplitudes of back reflection peaks originating from corneal interfaces that the in-depth OCT sensitivity stated in logarithmic scale changes almost linearly with the corneal depth. The mean value for the slope of this change can be estimated as $\mathrm{dS} / \mathrm{dz} \approx \pm$ 
$(1.45 \pm 0.15) \mathrm{V} / \mathrm{mm}$, where $\mathrm{z}$ ' is the depth of the beam's focal plane from the epithelium or endothelium surface. Thus, the "true" CDLBD can be constructed in two different ways. First, as it is shown in Figure 2B, where "true" CDLBD represents the envelope of the signals measured close to the focal planes of the probing beam. The second approach is by using only a single profile of the recorded CDLBD (Sexp) and correcting the measurements by $\mathrm{dS} / \mathrm{dz}$ ' according to relation (6):

$$
\operatorname{Sc}\left(z^{\prime}\right) \approx \operatorname{Sexp}\left(z^{\prime}\right)+\left(d S / d z^{\prime}\right) \cdot z^{\prime} .
$$

It should be noted that the results of this correction as shown in Figure $2 \mathrm{C}$ appear to be accurate since the systematical error that can propagate through the entire interval according to the measured uncertainty of $\mathrm{dS} / \mathrm{dz}$ ' will not exceed $5 \%$. 

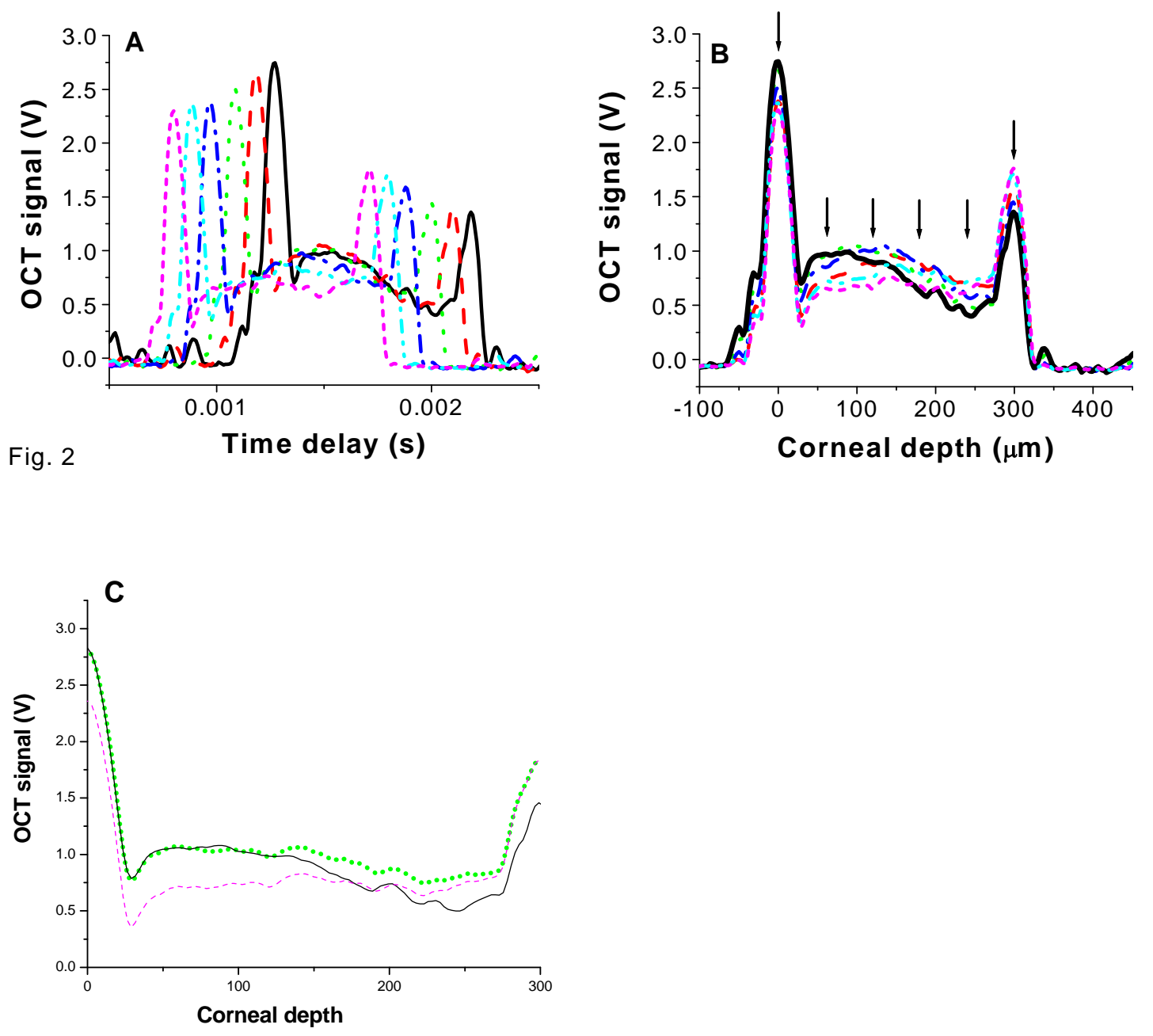

Figure 2. Reconstruction of the "true" corneal backscattering profiles (suppression of the effect of the confocal function); (A) The change of the corneal back reflection and backscattering due to the OCT probe displacement relative to the cornea (every step equals to about $40 \mu \mathrm{m}$ of the OCT probe axial shift), bold solid line - focusing on the epithelium layer, bold short dash line-focusing on the endothelium layer; (B) The "true" corneal backscattering profile (envelope of the maximum signals from different corneal depths from Fig. 2A), bold solid line - backscattering profile while focusing the probing beam on the epithelium surface, arrows are depicting the consequent positions of the focused beam focal planes inside the cornea; (C) The "true" corneal backscattering profile (short dotted line) derived after making correction by using relation (6). For comparison CDLBDs recorded with beam focused on the epithelium surface (solid line) and on the endothelium layer (dashed line) are presented. 


\subsection{Hydration manipulation results}

The results presented in Figures 3 and 4 exhibits the effects of hydration manipulation on the corneal backscattering after topical applications of water and dextrose, respectively. Figure 3 shows six plots for the backscattering distribution within the rabbit cornea recorded at six consecutive time intervals. One profile was obtained prior to the perturbation while the following 5 profiles were obtained during the first 4 minutes (at 5 different time intervals) after application of one drop of water on the epithelial surface. Figure 4 depicts the profiles of the backscattering distribution within the rabbit cornea at five consecutive time points, before and after inducing local dehydration of the cornea by exposing the epithelial surface to one drop of dextrose $(50 \%)$. Note the strong peak observed near the endothelial region of the cornea (shown by arrow) is due to artifacts inherent to the instrumentation used for OCT imaging in this study.

Figures $3 \mathrm{~A}$ and $4 \mathrm{~A}$ depict the enveloping prominent peaks with constant amplitude (for each series) due to back reflection originated from either epithelial or endothelial interface. These peaks were used as references to ensure the absence of optical distortion in measured CDLBDs caused by curvature of the drop of liquid that is applied to the surface of the cornea. As exhibited in Figures $3 \mathrm{~A}$ and $4 \mathrm{~A}$, the amplitude of the reference peaks were maintained constant while a considerable decrease or increase was induced in the amplitude of the backscattering profile within the first $200 \mu \mathrm{m}$ of the cornea (from the epithelium layer) following topical application of one drop of water or dextrose, respectively. Thus, the relative decrease in the backscattering amplitude (Figure $3 \mathrm{~B}$ ) at a depth of $0.1 \mathrm{~mm}$ from the epithelium/air interface can be estimated as $\Delta \mathrm{S} / \mathrm{S} 0 \approx-0.21$. In contrast to the response of the cornea to hypotonic-based perturbation (Figure 3), treatment of the cornea with a hypertonic agent in Figure 4 (dehydration) induced an increase in the backscattering amplitude over the same depth and after approximately the same time interval. This increase has a value of approximately $0.17 \mathrm{~V}$ from the initial amplitude of $0.64 \mathrm{~V}$ that gives $\Delta \mathrm{S} / \mathrm{S} 0 \approx+0.27$. This means that the total range of the corneal backscattering signal change during these hydration/dehydration processes can be estimated at $48 \%$.

It should be noted that during hydration/dehydration process the corneal thicknesses remain approximately constant (Figures $3 \mathrm{~A}$ and $4 \mathrm{~A}, \Delta \mathrm{L} / \mathrm{L} 0 \leq 0.05$ ) and, according to relations (3) and (4), the relative change of backscattering signal $\Delta \mathrm{S} / \mathrm{S} 0$ and corneal hydration $\Delta \mathrm{H} / \mathrm{H} 0$ must not be more than a few percents. This significant difference between $\Delta \mathrm{S} / \mathrm{S} 0$ estimated for the steady state corneal condition and its experimentally measured dynamic values changing in time across the cornea strongly suggest a correlation between the dynamic local change of $\Delta \mathrm{S} / \mathrm{S} 0$ and the corresponding dynamic local change in hydration due to water gradients that indeed exist in the cornea and spread through all corneal layers during hydration/dehydration processes. These observations further support our earlier suggestion for using relation (5) to quantitatively characterize the local hydration changes in corneal tissue as monitored by OCT. According to relation (5) the changes in local hydration, $\Delta \mathrm{H} / \mathrm{H} 0$ during the hydration or dehydration of cornea can be estimated to be approximately $\pm 15 \%$. 

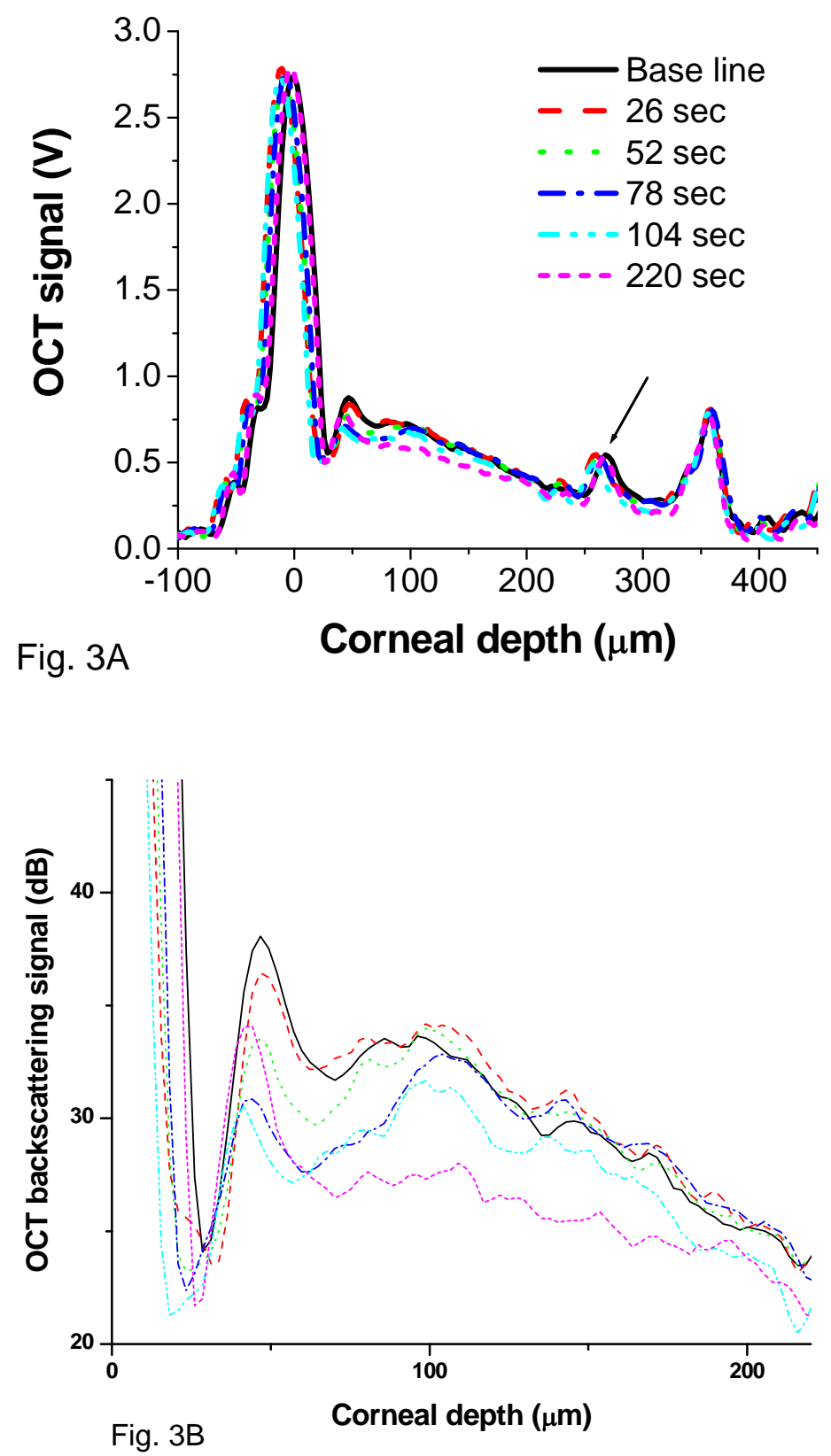

Figure 3. (A) The backscattering signals of the cornea recorded for six consecutive time intervals before and after applying one drop of water to the epithelial surface; solid line - before application of water, dash line - 26 sec after application of water, dot line - 52 sec after application of water, dash dot line$78 \mathrm{sec}$ after application of water, dash double dot line - $104 \mathrm{sec}$ after application of water, and short dash line - 220 sec after application of water; oblique arrow depicts the OCT optical artifact. (B) Magnified version of Fig. 3 A after correcting for OCT confocal function using relation (6). 


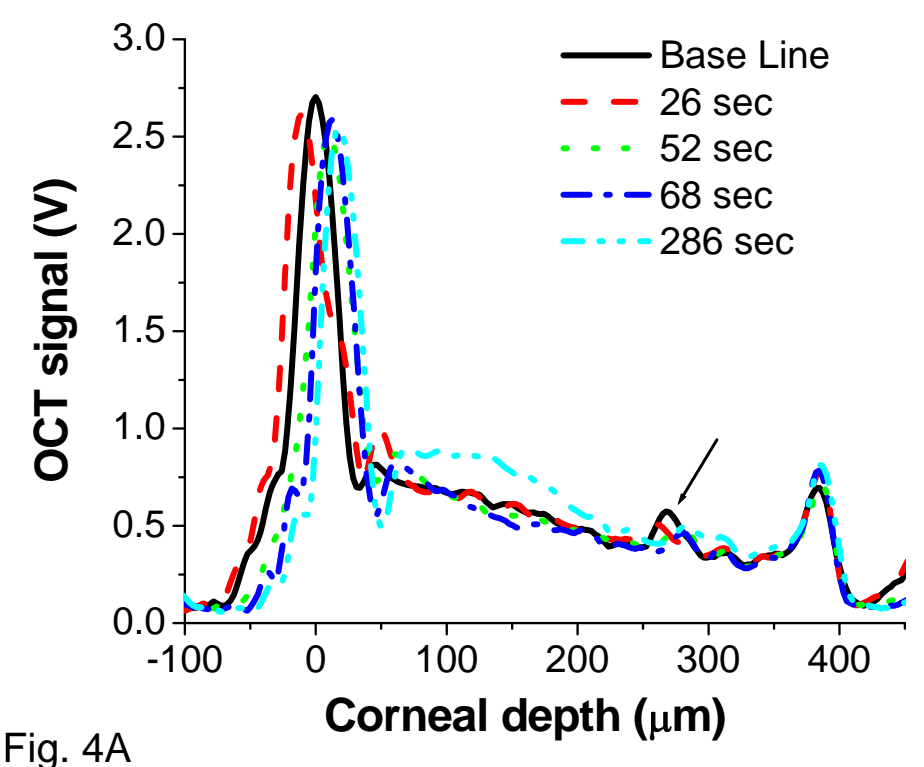

Fig. 4A

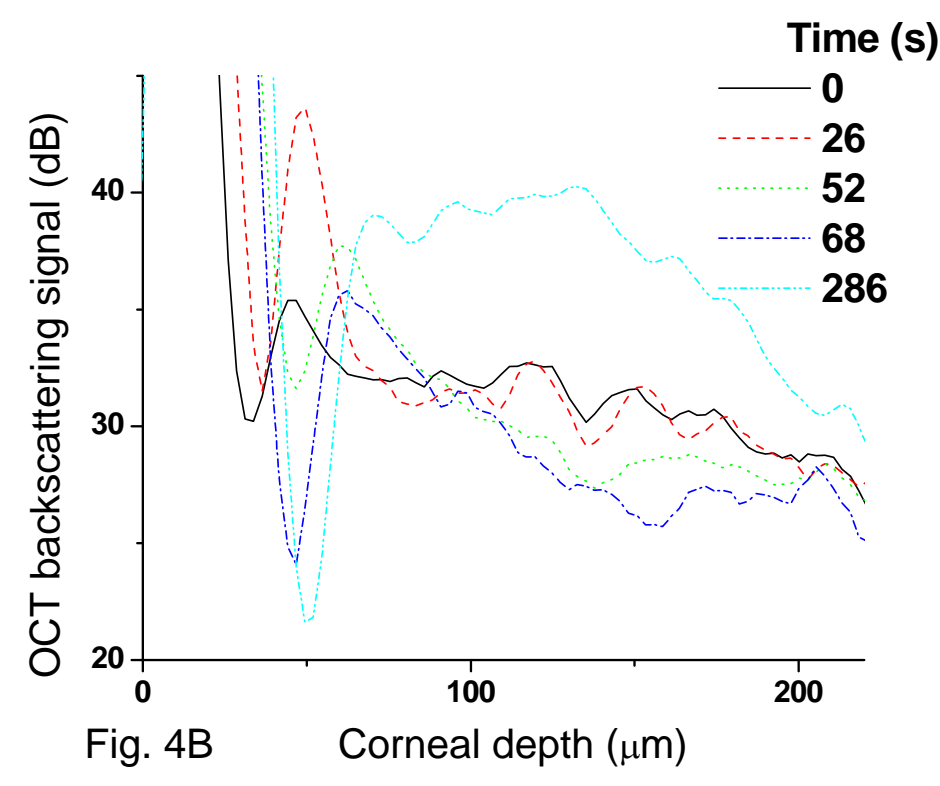

Figure 4. (A) The backscattering signals of the cornea recorded for five consecutive time intervals before and after applying one drop of dextrose (50\%) to the epithelial surface; solid line - before application of dextrose, dash line - 26 sec after application of dextrose, dot line - 52 sec after application of dextrose, dash dot line - $68 \mathrm{sec}$ after application of dextrose, and dash double dot line$286 \mathrm{sec}$ after application of dextrose; oblique arrow depicts the OCT optical artifact. (B) Magnified version of Fig. $4 A$ after correcting for OCT confocal function using relation (6). 
We conclude that the temporally measured CDLBDs provide the opportunity to gauge the rate of water movement inside the cornea. To estimate this rate more accurately we re-plotted and magnified the CDLBDs depicted in Figures $3 \mathrm{~A}$ and $4 \mathrm{~A}$ and corrected these profiles by using relation (6) as shown in Figures $3 \mathrm{~B}$ and $4 \mathrm{~B}$. The depth of the altered backscatter intensity in the backscattering profile of the cornea in Figures 3B at 104 and 220 seconds after perturbation of the cornea corresponds to depths of 100 and $240 \mu \mathrm{m}$ from the corneal surface, respectively. Detectable dextrose-induced changes in the backscattering profile of the cornea can be observed (Figure 4B) as deep as $250 \mu \mathrm{m}$ from the epithelial surface, at 286 seconds after application of one drop of dextrose. Thus, for either corneal hydration or dehydration, the rate of the perturbation spreading across the cornea can be estimated as $1 \mu \mathrm{m} / \mathrm{s}$ with an uncertainty of approximately $30 \%$.

\section{Discussions}

While applications of OCT for imaging of the corneal morphology and precise measurements of CCT offer new opportunities for diagnosis of corneal diseases, functional studies of the cornea using OCT may require quantitative assessment of temporal and spatial changes in the optical properties of the cornea following perturbation of cornea hydration or during pathological changes ${ }^{9,21,22}$. For these applications, it becomes essential to identify the origin of the changes that may occur in the back reflection and especially the backscattering profile of light within the cornea. Using reliable calibration techniques and accurately quantifying the backscattering profile form the essential steps to assess the changes in the corneal morphology and perform functional studies of the cornea using $\mathrm{OCT}^{13}$.

An important step for accurate measurement of the backscattering with OCT is speckle noise reduction ${ }^{23,24}$. The degree of the reduction in speckle noise is nearly related to the square root of the number of spatially independent lateral scans used for averaging. This means that to reduce the speckle noise by a factor of 10, one must acquire about 100 lateral scans. Results depicted in Figures 1B and $1 \mathrm{D}$ emphasizes the importance of filtering speckle noise in order to eliminate this source of distortion.

Potential applications of OCT for quantitative imaging of the cornea must account for the error that may be caused by the OCT sensitivity dependence on depth. When the OCT probing light with a highly focused beam is applied to perform noninvasive optical sectioning of the cornea, the OCT sensitivity does not remain constant along different corneal depths, but remarkably changes with the change in distance between the focal plane and the region of interest. For a corneal thickness of $0.5 \mathrm{~mm}$, the difference between the scattering signals when the beam is focused on the epithelium or the endothelium surface can be comparable with or even exceeds the magnitude of scattering signal itself. Thus, correction of the signals for this confocal function is vital for an accurate quantitative measurement of the backscatter light. For the case when the OCT 
probing beam is focused on the epithelium/air interface, one could easily correct the distortion present in the acquired scan by using the priory measured confocal function. Considering the fact that light attenuation due to the scattering and absorption is very low in the cornea, the envelop of the backscattering signals, as depicted in Figure 2B, is a true representation of the actual distribution of backscattering intensity within the cornea in logarithmic scale. The extrapolation of these results ${ }^{25}$ suggest an approximate attenuation coefficient of $\mu \mathrm{t} \cong 0.03-0.1$ $\mathrm{mm}-1$ for the cornea at $\lambda=1.3 \mu \mathrm{m}$.

The immediate change observed in the backscattering profiles after drops application along with the spreading of this change through the cornea in time, and the opposite direction of signal change between hypotonic and hypertonic applied agents, clearly demonstrate the utilization of the backscattering signal in enabling the water movement visualization inside the cornea. It is important to note that this water movement visualization within the cornea was realized while the total corneal thickness and therefore its total hydration were not changing. This was concluded by observing the significant change of local backscatter magnitude close to $\pm(20-25) \%$ (estimated local hydration change $\Delta \mathrm{H} / \mathrm{H} 0 \approx \pm$ $15 \%$ ), at the time that the total corneal hydration remained mainly unchanged (change in total corneal thickness equals less than 5\%).

It is interesting to note that the velocity of the perturbation spreading was estimated at approximately $1 \mu \mathrm{m} / \mathrm{s}$. This value is well under the limit of precision that can be realized for the measurements of the velocity by the dynamic light scattering technique (that is based on measurements of fluctuation in the backscattered light intensity of moving particles due to the Doppler effect), which uses roughly the same OCT technique ${ }^{26-29}$. This limit was measured by Izzat 27 et al. as $0.6 \mathrm{~mm} / \mathrm{s}$ through intact skin of hamster and it was estimated by us as tens of $\mu \mathrm{m}$ per second for polystyrene spheres suspension ${ }^{28,29}$. This emphasizes the capturing of the spatial aspect for hydration gradient change resulted by drops application in perturbed cornea.

In comparison, Raman Spectroscopy ${ }^{1,7}$ was also shown to be capable of quantifying the water gradient in the cornea, nevertheless the eye-safety and the practicality inherent to clinical applications of OCT makes it a far more attractive alternative. In addition, Raman Spectroscopy fails to probe the whole corneal thickness at a time when this is readily feasible with the OCT.

Previous in vitro corneal hydration studies have established the general view of a prominent role for the endothelial layer in regulating active fluid transport while a smaller role was assigned to the epithelial layer in transporting fluid across the cornea ${ }^{30}$. At the same time, other studies have also described the relatively large osmotic and diffusion permeability's found in the epithelium ${ }^{31-33}$, an active role for corneal thinning process by the epithelium ${ }^{6,34}$, and the existence of water channels inside the epithelium ${ }^{35,36}$, which all suggest a perhaps greater role for the fluid transport across the corneal epithelial layer than initially assumed.

Furthermore, the current view among experts supports the notion of a larger difference in the rate of fluid transport between the in vivo and in vitro conditions for the corneal epithelium. Yang et al. provided comprehensive 
evidence in this regard by demonstrating the transport of fluid across cultured corneal epithelial cell layers at a far greater rate than was previously thought6. Also, it appears that the $\mathrm{Cl}$ - transports across the epithelium is under neurophysiologic control ${ }^{37}$, which is vigorously severed under the in vitro conditions. These studies collectively suggest that the in vitro results for the transepithelial fluid transport may indeed be further apart from the actual in vivo conditions, as argued by Candia ${ }^{38}$. In the past, most of the studies have utilized either the unidirectional/diffusional flow with tritiated water $(3 \mathrm{H} 2 \mathrm{O})$ or net water flow by volumetric/gravitational procedures ${ }^{38}$. Again, both techniques can only be deployed for the "in vitro" conditions. A noninvasive tool capable of quantifying the fluid transport across the epithelium and within the corneal layers in vivo could elucidate the true transport capability and function of the corneal epithelium and the cornea as a whole. Furthermore, it is technically possible to non-invasively assess water transport across the endothelial layer by altering the endothelium pump activity using specific pharmacological agents. This could lead to the development of a noninvasive hydration monitoring system capable of detecting the fluid flow in vivo and ultimately assessing the dynamic function of the corneal endothelium. This mode of hydration monitoring may also be useful in "Penetrating Keratoplasty" practices, which holds the record number of transplant procedures in North America ${ }^{39,40}$. In cornea transplantation, the health and function of the cornea can have an immense impact on the rate of the graft failure in as many as $25 \%$ of the reported cases ${ }^{41}$. A non-invasive hydration monitoring system capable of detecting the fluid flow could help to assess the dynamic function of the corneal endothelium rather than the current traditional static function predictions, which takes place by counting the endothelial cells under a microscope to select the suitable donor cornea.

\section{Conclusions}

We demonstrated the feasibility of using OCT to quantitatively assess fluid transport across the corneal epithelium in vivo by monitoring the dynamics of hydration-induced changes in the spatial and temporal in-depth distribution of backscatter intensity.

This promising capability of OCT imaging was realized by recognizing the dependence of in-depth backscatter intensity on the extend of corneal hydration and by minimizing the degree of distortions in OCT signal induced by sources such as speckles, confocal function and the curvature of the cornea. In summary, the experimental results of the current study demonstrate the feasibility for application of OCT for in vivo noninvasive measurements of water movement within the cornea. 


\section{Acknowledgments}

The authors would like to thank the following members of the Center for Biomedical Engineering: Irina Petrova, Kirill Larin and Brent Bell for their useful assistance. This study was supported by NSF grant - BES-0528986. 


\section{References}

1. N.J. Bauer, J.P. Wicksted, F.H. Jongsma, W.F. March, F. Hendrikse, M. Motamedi, "Noninvasive assessment of the hydration gradient across the cornea using confocal Raman spectroscopy," Invest. Ophthalmol. Vis. Sci. 39, 831-835 (1998).

2. M. Pircher, E. Götzinger, R. Leitgeb, A.F. Fercher, C.K. Hitzenberger, "Measurement and imaging of water concentration in human cornea with differential absorption optical coherence tomography," Opt. Express 11, 21902197 (2003).

3. S.D. Klyce, "Enhancing fluid secretion by the corneal epithelium," Invest. Ophthalmol. Vis. Sci. 16, 968-973 (1977).

4. O.A. Candia, A.C. Zamudio, "Chloride-activated water permeability in the frog corneal epithelium,” J. Membr. Biol. 143, 259-266 (1995).

5. M.R. O'Neal, K.A.Polse, "In vivo assessment of the mechanisms controlling corneal hydration,” Invest. Ophthalmol. Vis. Sci. 26, 849-856 (1985).

6. H. Yang, P.S. Reinach, J.P. Koniarek, Z. Wang, P. Iserovich, J. Fischbarg, "Fluid transport by cultured corneal epithelial cell layers," Br. J. Ophthalmol. 84,199-204 (2000).

7. B.T. Fisher, K.A. Masiello, M.H. Goldstein, D.W. Hahn, "Assessment of transient changes in corneal hydration using confocal Raman spectroscopy," Cornea 22, 363-370 (2003).

8. A.S. Neubauer, S.G. Priglinger, M.J. Thiel, C.A. May, U.C. Welge-Lussen, "Sterile structural imaging of donor cornea by optical coherence tomography," Cornea 21, 490-494 (2002).

9. C. Wirbelauer, J. Winkler, G.O. Bastian, H. Haberle, D.T. Pham, "Histopathological correlation of corneal diseases with optical coherence tomography," Graefes Arch. Clin. Exp. Ophthalmol. 240, 727-734 (2002).

10. Y. Feng, J. Varikooty, T.L. Simpson, "Diurnal variation of corneal and corneal epithelial thickness measured using optical coherence tomography," Cornea 20, 480-483 (2001).

11. Y.L. Kim, J.T. Walsh Jr., T.K. Goldstick, M.R. Glucksberg, "Variation of corneal refractive index with hydration,” Phys. Med. Biol. 49 (5), 859-868 (2004).

12. B.O. Hedbys and S. Mishima, "The thickness-hydration relationship of the cornea," Exp. Eye Res. 5, 221-228 (1966). 
13. K. Hosseini, A.I. Kholodnykh, I.Y. Petrova, R.O. Esenaliev, F. Hendrikse, M. Motamedi, "Monitoring of Rabbit Cornea Response to Dehydration Stress by Optical Coherence Tomography," Invest. Ophthalmol. Vis. Sci. 45, 2555-2562 (2004).

14. Kholodnykh, K. Hosseini, I. Petrova, R.O. Esenaliev, M. Motamedi, "In vivo OCT assessment of rabbit corneal hydration and dehydration," Proc. SPIE 4956, 295-298 (2003).

15. S.N. Roper, M.D. Moores, G.V. Gelikonov, F.I. Feldchtein, N.M. Beach, M.A. King, V.M. Gelikonov, A.M. Sergeev, D.H. Reitze, "In vivo detection of experimentally induced cortical dysgenesis in the adult rat neocortex using optical coherence tomography," J. Neurosci. Methods. 80 (1), 91-98 (1998).

16. A.I. Kholodnykh, I.Y. Petrova, K.V. Larin, M. Motamedi, R.O. Esenaliev, "Precision of measurement of tissue optical properties with optical coherence tomography," Appl. Optics 42, 3027-3036 (2003).

17. Kholodnykh, K. Hosseini, I. Petrova, R.O. Esenaliev, M. Motamedi, "In vivo OCT assessment of rabbit corneal hydration and dehydration," Proc. SPIE 4956, 295-298 (2003).

18. A.I. Kholodnykh, I.Y. Petrova, M. Motamedi, R.O. Esenaliev, “Accurate Measurement of Total Attenuation Coefficient of Thin Tissue with Optical Coherence Tomography,” IEEE JSTQE 9, 210-221 (2003).

19. J.M. Schmitt, A. Knuttel, A. Gandjibakhche, and R. Bonner, "Optical characterization of dence tissues using low-coherence interferometry," in Holography, Interferometry, and Optical Pattern Reconstruction III, H. Podbielska, ed., Proc. SPIE 1889, 197-211 (1993).

20. J.A. Izatt, M. R. Hee, G.M. Owen, E.A. Swanson, and J. G. Fujimoto, "Optical coherence microscopy in scattering media," Opt. Lett. 19, 590-592 (1994).

21. C. Wirbelauer, C. Scholz, H. Hoerauf, G.O. Bastian, R. Engelhardt, R. Birngruber, H. Laqua, "Examination of the cornea using optical coherence tomography," Ophthalmologe 98 (2), 151-156 (2001).

22. C. Wirbelauer, C. Scholz, H. Haberle, H. Laqua, D.T. Pham, "Corneal optical coherence tomography before and after phototherapeutic keratectomy for recurrent epithelial erosions (2)," J. Cataract. Refract. Surg. 28, 1629-1635 (2002).

23. A.I. Kholodnykh, I.Y. Petrova, M. Motamedi, R.O. Esenaliev, “Accurate Measurement of Total Attenuation Coefficient of Thin Tissue with Optical Coherence Tomography,” IEEE JSTQE 9, 210-221 (2003). 
24. M. Pircher, E. Götzinger, R. Leitgeb, A.F. Fercher, C.K. Hitzenberger, "Measurement and imaging of water concentration in human cornea with differential absorption optical coherence tomography," Opt. Express 11, 21902197 (2003).

25. R.A. Farrell, R.L. McCally, P.E. Tatham, "Wave-length dependencies of light scattering in normal and cold swollen rabbit corneas and their structural implications," J. Physiol. 233, 589-612 (1973).

26. R.R. Ansari, "Ocular static and dynamic light scattering: a noninvasive diagnostic tool for eye research and clinical practice," J. Biomed. Opt. 9 (1), 22-37 (2004).

27. J.A. Izatt, M.D. Kulkarni, S. Yazdanfar, J.K. Barton, A.J. Welch, “ In vivo bidirectional color Doppler flow imaging of picoliter blood volumes using optical coherence tomography," Opt. Lett. 22 (18), 1439-1441 (1997).

28. D.P. Dave and T.E. Milner, "Doppler-angle measurement in highly scattering media," Opt. Lett. 25 (20), 1523-1525 (2000).

29. X.J. Wang, T.E. Milner, J.S. Nelson, "Characterization of fluid flow velocity by optical Doppler tomography,” Opt. Lett. 20 (11), 1337-1339 (1995).

30. S.D. Klyce, "Transport of $\mathrm{Na}, \mathrm{Cl}$, and water by the rabbit corneal epithelium at resting potential,” Am. J. Physiol. 228,1446-1452 (1975).

31. S. Mishima, B.O. Hedbys. "The permeability of the corneal epithelium and endothelium to water,” Exp. Eye Res. 6,10-32 (1967).

32. M. Parisi, O. Candia, L. Alvarez, "Water permeability of the toad corneal epithelium: the effects of $\mathrm{pH}$ and amphotericin B," Pflugers Arch. 383, 131-136 (1980).

33. J. Fischbarg, R. Montoreano, "Osmotic permeabilities across corneal endothelium and antidiuretic hormone-stimulated toad urinary bladder structures," Biochim. Biophys. Acta. 690, 207-214 (1982).

34. S.D. Klyce, C.E.Crosson, "Transport processes across the rabbit corneal epithelium: a review," Curr. Eye Res. 4, 323-331 (1985).

35. T. Horwich, C. Ibarra, P. Ford, A. Zamudio, M. Parisi, O.A. Candia, “ mRNA from frog corneal epithelium increases water permeability in Xenopus oocytes. Invest. Ophthalmol. Vis. Sci. 36, 2772-2774 (1995).

36. F. Kang, K. Kuang, J. Li, J. Fischbarg, "Cultured bovine corneal epithelial cells express a functional aquaporin water channel," Invest. Ophthalmol. Vis. Sci. 40, 253-257 (1999). 
37. S.D. Klyce, R.K. Wong, "Site and mode of adrenaline action on chloride transport across the rabbit corneal epithelium," J. Physiol. 266, $777-799$ (1977).

38. O.A. Candia, "Electrolyte and fluid transport across corneal, conjunctival and lens epithelia," Exp. Eye Res. 78, 527-535 (2004).

39. Eye bank association of America: 1996 Eye Banking Statistical Report. Washington, DC: Eye Bank Association of America, 1996.

40. United Network for Organ Sharing: 1996 Annual Report. Richmond, VA: United Network for Organ Sharing, 1996.

41. Price FW, Whitson WE, Collins KS, et al. Five-year corneal graft survival: a large, single-center patient cohort. Arch Ophthalmol 1993; 111:799-805. 
Chapter 9

Summary and conclusions 


\section{Summary and conclusions}

In the quest to prevent visual impairment or blindness, noninvasive and real-time monitoring of the available drugs in the ocular tissue plays a key role. Furthermore, the exact mode of transport for many ocular drugs and chemical compounds in the ocular tissues is still far from understood. In this thesis, two applicable noninvasive and optical-based diagnostic techniques in ophthalmology are studied. The first technique is Raman spectroscopy. Raman spectroscopy is the measurement of the wavelength and intensity of inelastically scattered light from molecules. The Raman scattered light occurs at wavelengths that are shifted from the incident light by the energies of molecular vibrations. Raman spectroscopy provides quantitative and qualitative information of molecules such as the drugs used for the treatment of intraocular inflammations. The second technique is Optical Coherence Tomography (OCT), also a laser-based noncontact and noninvasive imaging technique that is capable of obtaining highresolution images of the tissues. Both Raman spectroscopy and OCT add specific quantitative and qualitative information that cannot be obtained from the variety of already used optical examination modalities. In contrast to the commonly applied techniques, these new applications (can) work in a non-imaging mode. Although OCT was designed originally as an imaging device, it can deliver quantitative information such as degree of hydration in corneal tissue in a nonimaging mode in a so-called 'A-scan', as shown in this thesis. The same can be performed with Raman spectroscopy, however, it takes more time and provides a smaller spatial coverage at a time in a given sample.

The optical-based systems offer non-contact and accurate means of information collection and hence they essentially do not disrupt the physiology and morphology of the cells and tissues. These properties allow them to be used in a sensitive organ such as the eye, where the total tissue accessible for any type of probing is typically restricted.

Another property of the optical-based devices in data acquisition is related to their real-time capability. A fast and reliable optical data collection technique eliminates the time consuming steps that are involved with the more conventional biopsy and analytical methods, which are applied to gauge possible pathology and morphological or biochemical changes. This facet not only speeds up the availability of care in case of handling the pathology, but also provides the opportunity to study the biological processes as they occur without interference or under the influence of external interventions by either surgical procedures or drugs.

As mentioned in this thesis, data regarding intraocular drug concentration after injection and/or release from intravitreal implants are not assessable noninvasively. High concentrations of many intravitreal drugs are toxic and low concentrations miss the therapeutic threshold. Some of these drugs do 'leak' into the anterior segment of the eye and become detectable in the anterior chamber. However, currently there is limited understanding about these intraocular transport dynamics. The need for the above mentioned data becomes more 
prominent in the cases where re-administration of a drug is considered. In other words, the decision to administer a new dose of a drug can safely be made once the physician is confident that there is not much drug left in the ocular tissue from a previous administration that could potentially become toxic as the result of too much drug in proximity of sensitive ocular tissues. The current practical way of measuring the amount of the drug left in the eye is by paracentesis (conventional biopsy with a needle). This thesis proposes Raman spectroscopy as a powerful tool for a non-contact and noninvasive method of drug concentration analysis in the eye. Using Raman spectroscopy, drugs in the anterior chamber of the eye were successfully measured. In one experiment, the presence of a drug was adequately detected in the vitreous body of the eye.

Also in this thesis, the hydration of the cornea has been probed as a target for optical biopsy. Hydration of the cornea and its spatial distribution harbors an important place within various fields of physiology and pathology of the human eye and its surgery, such as cornea transplantation, precision of "cut" in refractive surgery, exploring pathology of corneal dystrophy, effects of topical drugs on corneal endothelial pump function and dynamics of the penetration of topical drugs through the corneal layers. Using OCT, efforts have been set to develop a system capable of monitoring and measuring the hydration distribution across the corneal layers both in time and in space on a noninvasive and eye-safe basis. Emphasis has been put on practical aspects such as the much needed understanding of and addressing the potential sources of distortions of the light backscattering as a crucial step to acquire comparable data and to produce reliable images. Furthermore, it was demonstrated that in vivo corneal light backscatter monitoring by OCT could be used to visualize the movement of water or potentially topical drugs across the cornea in real-time ${ }^{1}$.

The modifications necessary to use these techniques successfully in ophthalmology and the forthcoming findings are discussed in this thesis. Chapters 2, 3 and 4 discuss the application of Raman spectroscopy in analyzing therapeutic compounds injected into the eye. Chapters 5, 6, 7 and 8 discuss OCT and its applicability to investigate the morphological changes caused by administered compounds to the eye. In the following text, the material discussed in each chapter is briefly explained.

Chapter 2 exhibits material in relation with the possibilities of Raman spectroscopy in monitoring of the antiviral drug Ganciclovir in vivo. Various known concentrations of Ganciclovir were successfully detected in the anterior chamber of rabbit eyes in a pilot study. The specific Raman signature of Ganciclovir was assessed at several concentrations close to therapeutic values. Spectral features sole to Ganciclovir were identified and distinguished from the background.

Chapter 3 extends the possibilities of Raman spectroscopy in the detection of the more common intravitreal drugs used for the treatment of intraocular inflammation. This chapter describes the noninvasive monitoring of the antifungal drug Amphotericin B and antibiotic drug Ceftazidime within the anterior chamber of the eye and in vivo condition. Compared to ocular tissue, unique spectral features of Ceftazidime were observed near 1028, 1506, 1586 and 
$1641 \mathrm{~cm}^{-1}$. Amphotericin B exhibited its characteristic peaks at 1156.5 and 1556 $\mathrm{cm}^{-1}$. The feasibility of Raman spectroscopy for the detection of Ceftazidime, at even a lower concentration than the therapeutic dose is demonstrated. Furthermore, Amphotericin B was successfully detected in another eye compartment, namely, in the vitreous space of the eye.

Chapter 4 explains how Raman spectroscopy was employed in detecting a clinically used biomaterial in the human eye. The amount of laser power to obtain a Raman spectrum was low enough such that a complete eye-safe detection of biomaterial was demonstrated. Silicon oil, which is commonly used in vitrectomy procedures, was successfully monitored at $2906 \mathrm{~cm}^{-1}$ and $2965 \mathrm{~cm}^{-1}$ in a human eye in vivo. This chapter confirms the practicality of Raman spectroscopy for use in humans.

Chapter 5 shifts the scope of this thesis toward the applications of OCT in the cornea. While other OCT studies lay the accent in visualization of the cornea and its anatomical structure, this work demonstrates how OCT signals from the cornea can be interpreted in a different manner so that a change in physiology can also be studied with this technique. The following chapters explore ways to investigate and quantify the backscattering signal in relation with the change in hydration state of the cornea resulted from the impact of a topical drug on the cornea. The study in chapter 5 was based on monitoring the effects of the hypertonic agent Ophthalgan and the subsequent changes introduced in the cornea on a qualitative basis. The same chapter also discusses the importance of some of the modification factors for the optical biopsy by OCT, such as the correction for the 'confocal function' and 'speckle noise' reduction.

Chapter 6 presents some new improved data about the correlation of corneal light backscattering in-depth distribution with the magnitude and direction of water gradient within the rabbit cornea in vivo. This is achieved during the deand rehydratation processes of the rabbit cornea in vivo. Also the relation of the amplitude of light backscattering and the corneal thickness is discussed. Unlike the well-known effect of the immediate increase of the corneal opacity with corneal swelling, a new effect, which is a primary decline of the amplitude of light backscattering in the anterior part of the stroma during the swelling process, is reported. This was achieved by spatial backscattering measurement across the entire corneal thickness and thus a backscattering gradient could be observed for different layers of the cornea in real-time. In addition to the modification factors named in the previous chapter, corrections for the corneal curvature that also affect OCT measurements are discussed.

Chapter 7 discusses the de- and rehydration of the rabbit corneas in vivo at a greater depth. The direct relation of the corneal thickness and hydration is further explored. The study findings indicated an inverse relation between the logarithm of the intensity of the backscatter within the cornea and the degree of corneal hydration at which the intensity of backscatter changed up to 20 times between the peak of de- and rehydration phases. This work also demonstrates how OCT can play an important role in real-time monitoring of physiological events in the cornea. After a severe dehydrating effect in the cornea caused by a strong dehydrating agent, an overshoot in the corneal rehydration and thickness is 
successfully monitored and quantified during the recovery of the cornea. Adjunctive formulas are offered to investigate the relationship between the corneal backscattering and its thickness.

In chapter 8 , the information presented in the previous chapter for the relation between the light backscattering in different corneal regions and the corneal hydration is further explored. The feasibility of OCT in assessing the dynamics of corneal hydration changes in real time, after topical application of either hypertonic or hypotonic agents by accurate measurements of corneal indepth light backscatter distribution (CDLBD) was investigated. Optical perturbation in CDLBD, induced by water movement in rabbit cornea due to corneal hydration or dehydration propagated through the cornea at an approximate rate of $1 \mu \mathrm{m}$ per second. This led to use the backscatter distribution within the cornea to detect the actual water movement within this ocular medium both in real-time and in vivo. Quantification means are also suggested to measure the exact amount of water displacement in time and space.

As partly shown in this thesis, one modus operandi is not sufficient to address all the lingering questions such as drug transport within ocular tissue and its effect on the morphology. Therefore, a combination of techniques seems to make a convincing case for the next generation of diagnostic devices. One could suggest a combination of the two techniques used in this thesis, namely, OCT and Raman spectroscopy to address the challenges mentioned above. Such a combination will need to address the inherent sensitivity that is further complicated by the various functions and limitations of the eye itself. Examples that need to be addressed are: the sensitivity of the retina to radiation damage, the rapid dehydration of the cornea while exposed to the air, the focusing and refractive properties of the anterior segment optics and the eye movements.

\section{Future}

The noninvasive, non-contact and real-time property of Raman spectroscopy along with its high molecule (in this thesis: drug) sensitivity and specificity, advocates its further application as a strong optical biopsy tool. Traditionally, limitations of Raman spectroscopy such as the relative high laser power needed for a detectable Raman signal for some molecules (e.g. glucose concentration within physiological ranges) and a relative poor signal to noise ratio were cited as challenges for an immediate use of this system using the conventional and off-the-shelf devices. Fortunately, steps have been taken to improve these issues in recent years. Better optical setups are being developed and near infrared (NIR) light sources are more progressively used to address the eye safety issues associated with shorter wavelengths. Also, better multivariate analysis along with more sophisticated software for a better signal-to-noise processing are being developed and utilized. In addition, new optical probes are being constructed to divert the probing light ray such that it would not directly irradiate the retina and would pass through the anterior chamber of the eye at different incoming and outgoing angles. The development around Raman 
technology does not stop there. Other exciting Raman related techniques such as Coherent Anti-Stokes Raman Spectroscopy (CARS), Raman Induced Kerr Effect Spectroscopy (RIKES), Inverse Raman Spectroscopy (IRS), Stimulated Raman Gain Spectroscopy, Photoacoustic Raman Spectroscopy (PARS) are being developed at a much higher rate than where the technology was a decade ago.

On the OCT front, many exciting and recent developments are also visible. New light sources such as Ti:Sapphire- and Nd:Glass femtosecond lasers are enhancing the axial resolution to the level of single digit micrometers (Nd:Glass $5 \mu \mathrm{m}$ and Ti:Sapphire $1 \mu \mathrm{m})$. Another interesting field involves the refinement of Spectroscopic Optical Coherence Tomography, which is still in its early years ${ }^{2}$. Using this technique, imaging of functional properties of tissues on a micron scale can be achieved. It utilizes the broad spectral bandwidth of the optical source to obtain information from the spectral content of the backscattered light. Doppler OCT catheters are being developed as an integrated functional and structural imaging modality. Also, novel imaging software is continuously being developed where three-dimensional imaging capabilities are advancing the state of the art.

As of now, OCT or Raman spectroscopy can only be applied to the eye diagnostic separately. The reason for this is the inherent difference of optics and principals used within these two operational systems but perhaps even more, because the capabilities of these two systems have been traditionally considered individually with either imaging purposes (OCT) or analytical intentions (Raman spectroscopy). One could argue the usefulness of combining these two techniques into one device, such that it could enable the probing of a certain region of the eye tissue. In that case, different types of imaging can be acquired from the probed region in the eye tissue, providing analytical and morphological information at the same time. As an example, one could envision an end-probe unit with adaptive optics (the housing and the focusing lens that focuses the light on the sample) mounted on a slit-lamp with a (dual) fiber optic connection to a Raman and an OCT system.

Combination of OCT with another imaging modality has already been achieved in recent years. The OCT/SLO is a combination system, which is unique in its ability to simultaneously produce a confocal Scanning Laser Ophthalmoscope (SLO) image and an Optical Coherence Tomography (OCT) image using a single source. Another commercially available system is a Spectral OCT/SLO combination that has scanning laser ophthalmoscope microperimetry; another system has built-in color fundus photography capability; and a fourth system provides color fundus photography, angiography, and fundus autofluorescence. All of these systems provide a rendered fundus image from the 3-D data set and eventually will allow importation of color photographs as overlays to the OCT image.

Given the emerging opportunities and enhancements of the optical based technologies, it is expected that optical biopsy in the eye will form a compelling and adjunct alternative for the diagnostic arsenal of the ophthalmologists and vision scientists, bringing personalized medicine one step closer to reality. 


\section{References}

1. Ghosn MG, Tuchin VV, Larin KV. Nondestructive Quantification of Analyte Diffusion in Cornea and Sclera Using Optical Coherence Tomography. IOVS. 2007;48:2726-2733.

2. Kaluzy BJ, Kaluzny JJ, Szkulmowska A et. al. Spectral optical coherence tomography: A novel technique for cornea imaging. Cornea. 2006;25(8):960-5. 


\section{Samenvatting en conclusies}

In de zoektocht naar de mogelijkheid om slechtziendheid of blindheid te voorkomen speelt niet invasieve diagnostiek en de mogelijkheid tot het vervolgen in real-time van stoffen en geneesmiddelen in het oog, een cruciale rol. Daarnaast is de exacte wijze van het transport in de oculaire weefsels van veel chemische verbindingen en geneesmiddelen nog onbekend. In dit proefschrift worden twee niet invasieve en optisch gebaseerde diagnostische technieken in de oogheelkunde bestudeerd. De eerste techniek is Raman spectroscopie. Raman spectroscopie is de meting van de golflengte en intensiteit van het inelastisch verstrooid licht dat door bepaalde moleculen worden uitgezonden nadat ze belicht zijn door monochromatisch licht. Het Raman effect treedt op door verschuiving van de golflengten van het invallend licht door de energieën van de moleculaire trillingen. Raman spectroscopie geeft kwantitatieve en kwalitatieve informatie van moleculen, zoals van geneesmiddelen die gebruikt worden voor de behandeling van ontstekingen in het oog. De tweede techniek is Optical Coherence Tomography (OCT), een laser gebaseerde non-contact en niet invasieve beeldvormende techniek die in staat is om hoge resolutie afbeeldingen van de weefsels te verkrijgen. Zowel Raman spectroscopie als de OCT geven specifieke kwantitatieve en kwalitatieve informatie die niet kan worden verkregen door andere gebruikte optische onderzoeks methoden. Hoewel de OCT oorspronkelijk was ontworpen als een beeldvormend techniek, kan het ook kwantitatieve informatie verkrijgen zoals de mate van hydratatie van het hoornvlies weefsel in een niet-beeldvormende-modus in een zogenaamd 'A-scan', hetgeen werd aangetoond in dit proefschrift. Hetzelfde kan worden uitgevoerd met Raman spectroscopie, maar het verschil is dat het meer tijd in beslag neemt en dat de informatie uit een kleinere ruimtelijke dimensie komt per bemonster tijdstip.

De optisch gebaseerde systemen bieden een non-contact en accuraat middel voor het verzamelen van informatie en verstoren de fysiologie en morfologie van de te onderzoeken cellen en weefsels niet. Deze eigenschappen zijn vooral van belang in een delicaat orgaan, zoals het oog, waarbij de weefsel toegankelijkheid beperkt is voor menige bemonsterings techniek.

Een andere eigenschap van de optisch gebaseerde technieken in data acquisitie betreft hun real-time mogelijkheden. Een snelle en betrouwbare optische techniek elimineert de tijdrovende stappen die betrokken zijn bij de meer conventionele biopsie en de analytische methoden die worden toegepast om pathologie en morfologie of biochemische veranderingen te meten. Deze eigenschap versnelt niet alleen de toegespitste behandeling van de ziekte, maar biedt ook de gelegenheid voor het bestuderen van de biologische processen zoals ze zich in vivo voordoen, zonder externe interventies zoals chirurgische ingrepen.

Zoals besproken in dit proefschrift zijn data betreffende de intraoculaire concentraties van een geïnjecteerd geneesmiddel en/of de vrijzetting van een geneesmiddel uit een intravitreaal geplaatst implantaat meestal niet meetbaar zonder het gebruik van invasieve methoden. Te hoge concentraties van intraoculaire geneesmiddelen kunnen schadelijk zijn terwijl te lage concentraties de therapeutische drempel missen. Sommige van deze 
geneesmiddelen 'lekken' vanuit het glasvocht naar de voorste oogkamer. Echter, momenteel is er weinig kennis over deze intraoculaire vloeistof dynamiek. De noodzaak om gegevens te hebben over intraoculaire concentraties van stoffen wordt dwingender in die gevallen waarbij overwogen moet worden een geneesmiddel opnieuw toe te dienen. Met andere woorden, de beslissing om het veilig kunnen toedienen van een nieuwe dosis van hetzelfde geneesmiddel kan worden verricht zodra de arts ervan overtuigd is dat de overgebleven spiegel in het oogweefsel uit een vorige dosering, laag genoeg is om toxiciteit te verhinderen. De huidige manier van meten van intraoculaire stoffen of geneesmiddelen in het oog is door een paracentese (conventionele biopsie met een naald). In dit proefschrift wordt Raman spectroscopie voorgesteld als een krachtig instrument voor een non-contact en niet invasieve meting van de concentratie van stoffen in het oog. Geneesmiddelen in de voorste oogkamer werden gemeten met behulp van deze techniek. Daarnaast werd de aanwezigheid van een geneesmiddel vastgesteld in het glasachtig lichaam van het oog.

In dit proefschrift wordt tevens de hydratatie van het hoornvlies bestudeerd als een geschikt doel voor optische biopsie. Hydratatie van het hoornvlies en de ruimtelijke verdeling ervan vormt een belangrijke plaats binnen de fysiologie en pathologie van het menselijk oog. De hydratatie speelt een cruciale rol bij operaties, zoals een transplantatie van het hoornvlies of bij refractieve chirurgie; bij het onderzoeken van de pathologie van hoornvlies aandoeningen; of onderzoeken naar de invloed van geneesmiddelen op de hoornvlies endotheel pompfunctie en de dynamiek van de penetratie van geneesmiddelen via de verschillende hoornvlies lagen. Op basis van OCT is een systeem ontwikkeld waarmee de hydratatie verdeling over de verschillende hoornvlies lagen zowel in de tijd en in ruimte op een niet invasieve manier bepaald kan worden. Er is nadrukkelijk gekeken naar mogelijke oorzaken van verstoringen in het terug verstrooide licht en deze op te lossen om de reproduceerbaarheid van de data te verhogen.

Er werd aangetoond dat de beweging van water of geneesmiddelen in het hoornvlies in real-time waargenomen kan worden ${ }^{1}$.

De aanpassingen die noodzakelijk zijn voor het gebruik van deze technieken in oogheelkunde en de bevindingen worden in dit proefschrift besproken. De hoofdstukken 2, 3 en 4 gaan over de toepassing van Raman spectroscopie en het analyseren van geneesmiddelen geïnjecteerd in het oog. De hoofdstukken 5, 6, 7 en 8 bespreken OCT en de toepasbaarheid daarvan voor het onderzoek naar de morfologische veranderingen in het hoornvlies veroorzaakt door toegediende geneesmiddelen. Elk hoofdstuk wordt in de volgende tekst kort toegelicht.

Hoofdstuk 2 behandelt de mogelijkheden van Raman spectroscopie in de detectie van het antivirale geneesmiddel ganciclovir in vivo. Diverse concentraties ganciclovir werden met succes gedetecteerd in de voorste oogkamer in ogen van konijnen waarbij de gemeten concentraties dicht bij de therapeutische waarden liggen.

Hoofdstuk 3 bespreekt de mogelijkheden van Raman spectroscopie bij het opsporen van de meest voorkomende intravitreale geneesmiddelen die worden gebruikt voor de behandeling van intraoculaire ontstekingen. Dit hoofdstuk beschrijft de niet invasieve in vivo bepaling van het anti-fungus geneesmiddel amfotericine $\mathrm{B}$ en het antibioticum 
ceftazidime in de voorste oogkamer. Unieke Raman signalen van ceftazidime werden waargenomen bij 1028, 1506, 1586 en $1641 \mathrm{~cm}^{-1}$. Amfotericine B toonde karakteristieke Raman signalen bij 1156.5 en $1556 \mathrm{~cm}^{-1}$. De haalbaarheid van Raman spectroscopie voor de detectie van ceftazidime, zelfs bij een lagere concentratie dan de therapeutische dosis wordt aangetoond. Hoofdstuk 4 beschrijft Raman spectroscopie bij de detectie van een klinisch gebruikt biomateriaal in het menselijk oog. De benodigde laser power voor het verkrijgen van een Raman spectrum was laag genoeg om een oog veilige detectie van het biomateriaal mogelijk te maken. Dit biomateriaal, siliconen olie, wordt gebruikt bij intra oculaire ingrepen waarbij het glasvocht vervangen wordt door siliconen olie. De stof specifieke Raman spectra werden waargenomen op $2906 \mathrm{~cm}^{-1}$ en $2965 \mathrm{~cm}^{-1}$. Dit hoofdstuk toont de bruikbaarheid van Raman spectroscopie bij de mens aan.

Vanaf hoofdstuk 5 verschuift het focus van dit proefschrift naar toepassingen van de OCT in het hoornvlies. Terwijl bij het reguliere OCT onderzoek het accent in de visualisatie van het hoornvlies en de anatomische structuur ligt, toont dit werk aan hoe OCT-signalen van het hoornvlies kunnen worden geïnterpreteerd op een spectroscopische manier, zodat een verandering in de fysiologie kan worden bestudeerd met deze techniek. De volgende hoofdstukken behandelen hoe de terugverstrooide signalen veranderen door de wijziging in hydratatie van het hoornvlies als gevolg van een lokaal toegediend geneesmiddel op het hoornvlies.

Hoofdstuk 5. Het onderzoek in dit hoofdstuk is gebaseerd op het volgen van de effecten van de hypertone geneesmiddel ophthalgan en de daaropvolgende wijzigingen in het hoornvlies op een kwalitatieve basis. In dit hoofdstuk wordt ook het belang besproken van aanpassingen die nodig zijn om een optische biopsie met OCT te verrichten, zoals de correctie voor de 'confocale functie' en 'speckle ruis' vermindering.

In hoofdstuk 6 wordt een aantal nieuwe gegevens over de correlatie van het hoornvlies licht terugverstrooiing en de betreffende diepte distributie met de omvang en richting van het water gradiënt in het hoornvlies van een konijn in vivo voorgesteld. Dit wordt bereikt tijdens de dehydratatie en rehydratatie behandelingen. Ook de relatie van de amplitude van licht terugverstrooiing en de dikte van de hoornvlies wordt besproken. In tegenstelling tot het bekende effect van de onmiddellijke toename van de hoornvlies troebeling bij hoornvlies zwelling, werd een nieuw effect waargenomen, dat een primaire daling liet zien van het amplitude van het terugverstrooide licht uit het voorste deel van het hoornvlies stroma tijdens het zwellings proces. Dit werd gerealiseerd door een ruimtelijke terugverstrooiings meting over de gehele dikte van het hoornvlies en zo werd de terugverstrooiings gradiënt waargenomen voor de verschillende lagen van het hoornvlies in real-time. Er worden ook correcties besproken die de invloed van de hoornvlies kromming, die gevolgen kan hebben voor de OCT metingen, opheft. Hoofdstuk 7 gaat verder met de directe relatie van de hoornvlies dikte versus hydratatie van het hoornvlies. De in dit hoofdstuk beschreven bevindingen tonen een inverse relatie aan tussen de logaritme van de intensiteit van de terugverstrooiing in het hoornvlies en de mate van hoornvlies hydratatie, waarbij de intensiteit van terugverstrooiing tot 20 maal kan veranderen. Dit werk toont ook aan hoe OCT een belangrijke rol kan spelen in realtime detectie van fysiologische processen in het hoornvlies. Na een fors dehydratatie effect in het hoornvlies veroorzaakt door een geneesmiddel, wordt de hoornvlies 
rehydratatie en de dikte veranderingen die daarmee gepaard gaan, met succes gevolgd en gekwantificeerd tijdens het hydratatie herstel van het hoornvlies.

Aanvullende rekenmodellen worden gegeven om de relatie te onderzoeken tussen de hoornvlies terugverstooiing en de dikte ervan.

In hoofdstuk 8 wordt de informatie die in het vorige hoofdstuk is besproken over de relatie tussen de licht terugverstooiing in verschillende hoornvlies lagen en de hoornvlies hydratatie nader onderzocht. De geschiktheid van de OCT bij de beoordeling van de dynamiek van de hoornvlies hydratatie veranderingen in de real-time, na lokale toediening van een hypotone of hypertone middelen, door nauwkeurige metingen van de zogenaamde hoornvlies diepte terugverstrooiing licht distributie (HDTLD) werd onderzocht. Optische storingen in HDTLD werden gedetecteerd als gevolg van water toeen afvoer in het hoornvlies. De water bewegingen in konijnen ogen werden gemeten met waarden rond de $1 \mu \mathrm{m}$ per seconde, veroorzaakt door hoornvlies de- en rehydratatie. De terugverstrooide licht distributie in het hoornvlies werd gebruikt voor het bepalen van de snelheid van de water bewegingen in real-time en in vivo. Kwantificering methoden worden voorgesteld voor het meten van de precieze omvang van waterverplaatsing in ruimte en tijd.

Zoals weergegeven in dit proefschrift is een enkele modus operandi niet voldoende om alle vragen zoals geneesmiddel transport binnen oogweefsel en de invloed daarvan op de morfologie te beantwoorden. Daarom lijkt een combinatie van technieken een logische stap in de ontwikkeling van de volgende generatie van diagnostische hulpmiddelen. Dit proefschrift beschrijft een combinatie van twee spectroscopische technieken, namelijk OCT en Raman spectroscopie. Een dergelijke combinatie zou een oplossing kunnen zijn om de inherente gevoeligheid van het oog zelf niet aan te tasten. Aandachtspunten zijn: de gevoeligheid van het netvlies voor licht schade, de snelle uitdroging van het hoornvlies blootgesteld aan de lucht, de refractieve eigenschappen van het oog, en de invloed van oogbewegingen op de metingen.

\section{Toekomst}

De niet invasieve, non-contact en real-time eigenschappen van Raman spectroscopie, gecombineerd met de hoge molecuul (in dit proefschrift: geneesmiddelen) sensitiviteit en specificiteit, pleiten voor een verdere toepassing van deze veelbelovende techniek als een optische biopsie methode. Van oudsher zijn de beperkingen van Raman spectroscopie, zoals de relatief hoge laser power die nodig is voor een detecteerbare Raman signaal voor bepaalde moleculen (bijvoorbeeld glucose concentratie in fysiologische reeksen) en een relatief slechte signaal / ruisverhouding, een drempel geweest voor onmiddellijk gebruik van dit systeem met een conventioneel off-the-shelf apparaat. Er zijn er stappen genomen in de afgelopen jaren om deze problemen aan te pakken. Betere optische opstellingen worden ontwikkeld en nabij-infrarood (NIR) lichtbronnen zijn geleidelijk meer gebruikt om de risico's van lichtschade die gepaard gaat met kortere golflengten te verminderen. Ook betere multivariate analyse modellen in combinatie met geavanceerde software voor een betere signaal / ruisverwerking worden ontwikkeld en benut. Daarnaast worden nieuwe optische instrumenten gebouwd om het excitatie licht dusdanig te richtten dat het niet rechtstreeks op het netvlies kan komen. De ontwikkeling rond de Raman technologie 
blijft niet beperkt tot het bovengenoemde. Andere uitdagende Raman technieken zoals Coherent Anti-Stokes Raman Spectroscopy (CARS), Raman spectroscopie Induced Kerr Effect (RIKES), Inverse Raman spectroscopie (IRS), Gain Gestimuleerde Raman spectroscopie, Photoacoustic Raman spectroscopie (PARS) worden ontwikkeld in een veel hoger tempo dan zo'n tien jaar geleden.

Ook op het OCT gebied, zijn er nieuwe en recente ontwikkelingen zichtbaar. Nieuwe lichtbronnen, zoals Ti: Sapphire-en Nd: Glas Femtoseconde lasers hebben de axiale resolutie verhoogd tot het niveau van enkele micrometers (Nd:Glass $5 \mu \mathrm{m}$ en Ti:Sapphire $1 \mu \mathrm{m})$. Een ander interessant gebied is de verfijning van de spectroscopische Optical Coherence Tomography, die zich nog in een vroeg stadium bevindt ${ }^{2}$. Met deze techniek kunnen functionele eigenschappen van weefsels op een micron schaal worden bereikt. Het maakt gebruik van de spectrale bandbreedte van de optische bron om informatie te verkrijgen uit het terugverstrooide licht. Doppler OCT catheters worden ontwikkeld als een geïntegreerd functionele en structurele beeldvormende modaliteit. Ook nieuwe beeldvormende software wordt voortdurend ontwikkeld om drie dimensionale imaging te realiseren.

Op dit moment kan OCT of Raman spectroscopie alleen worden toegepast op een afzonderlijk manier voor oog diagnostiek. De reden hiervoor is niet alleen het inherent verschil in optica en fysische grondslag, maar ook omdat de inzetbaarheid van deze twee systemen van oudsher beschouwd is als zijnde, voor beeldvormende doeleinden (OCT) dan wel voor analytische doeleinden (Raman spectroscopie). Men zou kunnen voorstellen deze twee technieken in een apparaat te combineren zodat analytische en morfologische informatie op hetzelfde moment verworven kan worden.

De combinatie van de OCT met andere beeldvormende technieken is reeds bereikt in de afgelopen jaren. De OCT/SLO is een combinatie systeem dat uniek in zijn vermogen is om tegelijkertijd een confocaal laser scanning ophthalmoscoop (SLO) beeld en een Optical Coherence Tomography (OCT) beeld te vormen met behulp van een enkele licht bron. Andere commercieel systemen zijn: een Spectral OCT/SLO combinatie met scanning laser oftalmoscoop microperimetry; terwijl een ander systeem een ingebouwde fundus fotografie met kleur mogelijkheid biedt; of een systeem dat kleur fundus fotografie, angiografie, en fundus autofluorescentie biedt. Al deze systemen maken een fundus afbeelding samengesteld uit de 3-D data set en uiteindelijk zal de invoer van kleuren foto's als overlays bij het OCT-afbeelding mogelijk worden.

Gezien de nieuwe mogelijkheden en verbeteringen van de optische technologieën wordt er verwacht dat een optische biopsie van het oog een aantrekkelijk en aanvullend alternatief vormt voor invasieve diagnostiek. Hierdoor kan de op de patiënt toegespitste geneeskunde een stap dichter bij de werkelijkheid komen. 


\section{References}

3. Ghosn MG, Tuchin VV, Larin KV. Nondestructive Quantification of Analyte Diffusion in Cornea and Sclera Using Optical Coherence Tomography. IOVS. 2007;48:2726-2733.

4. Kaluzy BJ, Kaluzny JJ, Szkulmowska A et. al. Spectral optical coherence tomography: A novel technique for cornea imaging. Cornea. 2006;25(8):960-5. 


\section{A few words of appreciation}

I am eternally thankful to the following people for their continuous support throughout my studies. Their encouragement and guidance have enabled me to finish this thesis.

I'd like to thank my parents for everything they did to help all their three children to receive a good education in a period when many others simply could not dream of such opportunities.

Dr. Roel J. Erckens,

Dear Roel, You have been helping me from the very first day when you travelled with me to Texas and taught me how to start the laser setup, all the way to the very end, helping me to edit this thesis with countless iterations. I was very fortunate to follow your work in the Raman lab at UTMB and it is an honor to have you as my co-promoter.

Professor Motamedi,

Dear Massud, I have a lot to thank you for. Your expertise and your continuous support have enabled me to finish my work. It has been a real pleasure and an honor to work under your leadership at UTMB.

Dr. Alexander Kholodnykh,

Dear Alex, Your arrival at UTMB was one of the luckiest events during my studies in Galveston. You taught me a great deal in optics and research in general, and it was your untiring work and expert efforts that guided me through some of the hardest works that we completed together. It is a privilege for any research fellow to work under your guidance.

My promoter, Professor dr. Hendrikse,

It has been several years now that I've had the pleasure of knowing you. You made a huge impact in my life by considering me for the continuation of your research collaboration abroad, granting me the opportunity to find a meaningful career. At any time that I was encountering a major roadblock, I could turn to you and you would be there to 'make things happen'. I am forever grateful for all your help and support.

I would like to express my appreciation to the honorable members of the "beoordeling commissie" for taking the time to review this thesis. Also, many thanks to Mr. Brent Bell at UTMB and Dr. Frans Jongsma at Maastricht University for their support throughout my studies.

Last but not least, I'd like to thank my dear sister Fariba and my beloved wife Delnaz for their unconditional support during the past several years. 


\section{Publications and Presentations}

- $\quad$ K.Hosseini, A.Kholodnykh, S.Y. Treschev, R.O. Esenaliev, F. Hendrikse, M.Motamedi. Noninvasive monitoring of water movement through rabbit cornea using Optical Coherence Tomography. Submitted.

- $\quad$ K. Hosseini, D. Matsushima, J. Johnson, G. Widera, K. Nyam, L. Kim, Y. Xu, Y. Yao, M. Cormier. Pharmacokinetic study of Dexamethasone disodium phosphate using intravitreal, subconjunctival and intravenous delivery routes in rabbits. J Ocul Pharmacol Ther. 2008 Jun;24(3):301-8.

- $\quad$ H. Cheong, M. Cormier, D. Matsushima, J. Johnson, G. Widera, K. Nyam, K. Hosseini. A cytotoxicity comparison study using beta-blockers on corneal epithelium and retinal pigment epithelium cell lines. Toxicol In Vitro. 2008 Jun;22(4):1070-6.

- $\quad$ D.M. Paskowitz, K.M. Donohue-Rolfe, H. Yang, K. Hosseini, C.M. Graybeal, G.C. Nune, D. Niculescu, M.A. Zarbin, M.M. LaVail, J.L. Duncan. "Neurotrophic Factors Minimize the Retinal Toxicity of Verteporfin Photodynamic Therapy". Investigative Ophthalmology and Visual Science. 2007;48:430-437.

- $\quad$ K.Hosseini, A.Kholodnykh, I.Petrova, R.O. Esenaliev, F. Hendrikse, M.Motamedi. Monitoring of Corneal Response to Dehydration Stress by Optical Coherence Tomography. Investigative Ophthalmology and Visual Science. 2004 Aug;45(8):2555-62.

- A.Kholodnykh, K.Hosseini, K.V. Larin, A.A. Vassilieva, R.O. Esenaliev, M.Motamedi. Accurate in vivo measurements of the corneal light backscattering in-depth distribution with OCT for different hydration states. SPIE proceedings 2004.

- $\quad$ K. Hosseini, F.H.M. Jongsma, F. Hendrikse, M. Motamedi. Noninvasive Monitoring of Commonly used Intraocular Drugs Against Endophthalmitis by Raman Spectroscopy. Lasers in Surgery and Medicine 2003 April Vol.32 (4).

- $\quad$ A.Kholodnykh, K.Hosseini, I.Petrova, R.O.Esenaliev, M.Motamedi. In vivo OCT assessment of rabbit corneal hydration and dehydration. SPIE proceedings 2003; BIOS, San Jose, CA, USA.

- $\quad$ R.J. Erckens, K.Hosseini, W.F.March, F.H.M Jongsma, J.P. Wicksted, H.K. Li, F.Hendrikse. Raman Spectroscopy: noninvasive determination of silicon oil in the eye. Potential applications for intraocular determination of biomaterials. Retina. 2002 December Vol.22 (6). 
- $\quad$ K.Hosseini, A.Kholodnykh, I.Petrova, R.O.Esenaliev, M.Motamedi. Noninvasive and Non-contact Assessment of Corneal Hydration in Real Time Using Optical Coherent Tomography. Proceedings of the Joint EMBS/BMES, Houston, TX, USA. October, 2002.

- $\quad$ K. Hosseini, W. March, F.H.M. Jongsma, F. Hendrikse, M. Motamedi. Noninvasive Detection of Ganciclovir in Ocular tissue by Raman Spectroscopy: Implication for Monitoring of Drug Release. J. Ocul. Pharmacol. Ther. 2002 Jun;18(3):277-85.

- $\quad$ K. Hosseini, D. Matsushima, J. Johnson, G. Widera, K. Nyam, L.Kim, Y. Xu, Y. Yao, M. Cormier. "Intravitreal, Subconjunctival, and Intravenous Delivery of Dexamethasone Disodium Phosphate in Rabbits; A Pharmacokinetic Study". ARVO, 2008. Fort Lauderdale, Florida.

- $\quad$ M. Cormier, H. I. Cheong, J. Johnson, K. Hosseini. "Cytotoxicity of several BetaBlockers in Human Retinal and Corneal Cell Lines". ARVO, 2008. Fort Lauderdale, Florida.

- $\quad$ K. Farinas, K. Hosseini, J. Lee, R. Padmanabhan. "Non-invasive Drug Delivery to the Back of the Eye Using Iontophoresis". Drug Device Research, ALZA Corporation, J\&J Excellence in Science Symposium, New Brunswick, New Jersey, November 6, 2006

- $\quad$ K.M. Donohue-Rolfe, D.M. Paskowitz, H. Yang, K. Hosseini, C.M. Graybeal, G.C. Nune, D. Niculescu, M.A. Zarbin, M.M. LaVail, J.L. Duncan. "BDNF Confers Long-Term Protection from the Retinal Toxicity of Verteporfin PDT". ARVO, 2006. Fort Lauderdale, Florida.

- $\quad$ C.P. De Moor, D. Edgren, K.Hosseini, R. Anderson. "Drug delivery concepts to target the back of the eye". ALZA Technology Summit 2005, Mountain View, California.

- J. Lee, K. Hosseini, A. Subramony, R. Padmanahban, K. Farinas, N. Cheruvu, U. Kompella. "In Vitro Simulation of Iontophoretic Ocular Drug Delivery “.ALZA Technology Summit 2005, Mountain View, California.

- $\quad$ K. Hosseini, K.G. Duncan, K. Donohue-Rolfe, R. Lowe, H. Yang, M.T. Matthes, D. Yasumura, M. M. LaVail, D.M. Schwartz, J. L. Duncan. "SR-BI and Apo E interactions affect dark adaptation in mice". ARVO, 2005. Fort Lauderdale, Florida.

- $\quad$ M. Motamedi, K. Hosseini, A. Kholodnykh, S. Treschev, R. Esenaliev, F. Hendrikse. "Noninvasive Optical Monitroing of Water Movement through Rabbit Cornea". ARVO, 2005. Fort Lauderdale, Florida.

- $\quad$ K.Hosseini, A.Kholodnykh, I.Petrova, R.O. Esenaliev, M.Motamedi. “Optical Coherence Tomography for in vivo High resolution Quantitative Imaging of the Corneal Function". ARVO, 2003. Fort Lauderdale, Florida. 
- $\quad$ K.Hosseini, A.Kholodnykh, I.Petrova, R.O. Esenaliev, M.Motamedi.

"Noninvasive and Non-contact Assessment of Corneal Hydration in Real-Time Using Optical Coherent Tomography". EMBS/BMES, 2002. Houston, Texas.

- $\quad$ K. Hosseini , F.H.M. Jongsma , F. Hendrikse ,M. Motamedi. "Noninvasive Detection of Intraocular Drug Concentration by Raman Spectroscopy". American Society for Laser, Medicine and Surgery 2002, Atlanta, Georgia.

- $\quad$ K. Hosseini, R,J. Erckens, W. March, F.H.M. Jongsma, F. Hendrikse, M. Motamedi. "Noninvasive assessment of Local Ceftazidime and Amphotericin B Concentration in ocular Tissue by Raman Spectroscopy". ARVO, 2002,Fort Lauderdale, Florida.

- $\quad$ K. Hosseini, R,J. Erckens, W. March, F.H.M. Jongsma, F. Hendrikse, M. Motamedi. "Niet-invasieve detectie van ganciclovir, amphotericin B en ceftazidime in vivo". NOG, 2002, Groningen, The Netherlands.

- $\quad$ K. Hosseini, W. March, F.H.M. Jongsma, F. Hendrikse, M. Motamedi.

"Noninvasive detection of ganciclovir in ocular tissue by Raman Spectroscopy". ARVO, 2001. Fort Lauderdale, Florida.

- $\quad$ K. Hosseini, W. March, F.H.M. Jongsma, F. Hendrikse, M. Motamedi, "Noninvasive assessment of local ceftazidime concentration in ocular tissue by Raman Spectroscopy". NOG, 2001. Maastricht, The Netherlands.

\section{Awards}

- $\quad$ Best Resident paper in the category "Basic Science" at the 22nd annual meeting of ASLMS. American Society for Laser, Medicine and Surgery, 2002 Atlanta, Georgia. 